\title{
REAL FORMS OF EMBEDDINGS OF MAXIMAL REDUCTIVE SUBALGEBRAS OF THE COMPLEX SIMPLE LIE ALGEBRAS OF RANK UP TO 8
}

\author{
WILLEM A. DE GRAAF AND ALESSIO MARRANI
}

\begin{abstract}
We give tables of noncompact real forms of maximal reductive subalgebras of complex simple Lie algebras of rank up to 8 . These were obtained by computational methods that we briefly describe. We also discuss applications in theoretical physics of these embeddings.
\end{abstract}

\section{INTRODUCTION}

The classification of maximal reductive subalgebras of exceptional and classical complex Lie algebras is well known. A systematic study started with the work of Dynkin Dyn52a, Dyn52b. Subsequently, it was developed in a number of works, such as [LG72, Min06, dG11]. For what concerns non-compact real forms, the maximal reductive subalgebras of exceptional and classical Lie algebras which give rise to symmetric embeddings are listed e.g. in Table 9.7 of Gil06, which reports results taken from a rather vast literature (see the references therein). On the other hand, the non-compact real forms of maximal, semisimple (reductive) subalgebras of exceptional Lie algebras which give rise to non-symmetric embeddings are studied in a number of papers scattered in works of mathematics and of theoretical physics, which are not easily collected; for instance, in the high-energy physics literature (Maxwell-Einstein-scalar theories, possibly endowed with some amount of local supersymmetry), some maximal subalgebras giving rise to non-symmetric embeddings have been considered in AFMT08, FMT12, FMZ13. In the mathematical literature Gray Gra72] classified the real forms of the maximal reductive subalgebras that arise as fixed point subalgebras of automorhisms of order three (overlooking two cases, see Section 3.1). Komrakov [Kom90 also classified all real forms of the maximal (non-symmetric) embedding $A_{4} \oplus A_{4} \subset E_{8}$, and he also carried out a classification of the maximal S-subalgebras of exceptional real Lie algebras.

Recently algorithms, together with their implementations, have been developed to compute the real forms of embeddings of complex semisimple Lie algebras, DFdG15, FdG15. However, a classificatory and exhaustive approach was not pursued in these papers. In the present paper, by developing and systematically applying these techniques we aim at obtaining the complete list of noncompact real forms of embeddings of maximal reductive subalgebras of the simple complex Lie algebras of ranks up to 8 .

Some remarks to clarify our aim are in order. Firstly, with a real form of an embedding of complex Lie algebras we mean a pair of real forms $\mathfrak{a}, \mathfrak{g}$ of complex reductive Lie algebras $\mathfrak{a}^{c}, \mathfrak{g}^{c}$ such that $\mathfrak{a}^{c} \subset \mathfrak{g}^{c}$ and $\mathfrak{a} \subset \mathfrak{g}$. Secondly, it is possible to have a pair of complex semisimple (or reductive) Lie algebras $\mathfrak{a}^{c} \subset \mathfrak{g}^{c}$ with real forms $\mathfrak{a}, \mathfrak{g}$ such that $\mathfrak{a}$ is maximal in $\mathfrak{g}$ without $\mathfrak{a}^{c}$ being maximal in $\mathfrak{g}^{c}$. In fact, Komrakov [Kom90] has a list of cases where that happens. We do not quite understand this list (for example, the cases appearing under item a) in the cited paper seem to be maximal reductive subalgebras, and also appear in our tables), nor do we have methods to detect those cases independently. Therefore we restrict to 
listing the real forms of embeddings of the maximal complex reductive subalgebras of complex semisimple Lie algebras.

The second part of the paper has the tables that we obtained containing the maximal reductive subalgebras of the noncompact simple real Lie algebras of rank up to 8 . These subalgebras are also contained, in explicit form, in the latest version of the CoReLG package for GAP4 ([DFdG19]).

The remainder of this paper is divided in a few sections. In Section 2 we describe the maximal reductive subalgebras of the simple complex Lie algebras. Section 3 gives an overview of some applications of real embeddings of reductive Lie algebras in high-energy theoretical physics. After that we have Section 4 giving a brief overview of the computational methods that we used to obtain real forms of complex embeddings. Finally Section 5 has the tables of those real forms.

\section{Maximal Reductive subalgebras of COMPlex Semisimple Lie} ALGEBRAS

In this section we describe the complex embeddings of which we compute the real forms.

Let $\mathfrak{g}^{c}$ be a complex simple Lie algebra. After Dynkin Dyn52b, a subalgebra $\mathfrak{a}^{c} \subset \mathfrak{g}^{c}$ is said to be regular if there is a Cartan subalgebra $\mathfrak{h}^{c}$ of $\mathfrak{g}^{c}$ with $\left[\mathfrak{h}^{c}, \mathfrak{a}^{c}\right] \subset \mathfrak{a}^{c}$. Such a subalgebra is spanned by root spaces with respect to $\mathfrak{h}^{c}$ along with $\mathfrak{h}^{c} \cap \mathfrak{a}^{c}$. A subalgebra of $\mathfrak{g}^{c}$ is called an $R$-subalgebra if it is contained in a proper regular subalgebra of $\mathfrak{g}^{c}$. A subalgebra that is not an R-subalgebra is called an S-subalgebra.

Dynkin showed that every non-semisimple subalgebra of $\mathfrak{g}^{c}$ is an R-subalgebra ([Dyn52b], Theorem 7.3). Hence S-subalgebras are necessarily semisimple. So the maximal reductive S-subalgebras coincide with the maximal semisimple Ssubalgebras. These have been classified by Dynkin Dyn52a, Dyn52b, and are also contained in the lists obtained in dG11.

Now we consider the regular maximal reductive subalgebras. Let $\Delta$ be a set of simple roots of the root system of $\mathfrak{g}^{c}$ with respect to a Cartan subalgebra $\mathfrak{h}^{c} \subset \mathfrak{g}^{c}$. Furthermore, let $\delta$ denote the highest root of the root system and write

$$
\delta=\sum_{\alpha \in \Delta} n_{\alpha} \alpha
$$

where the $n_{\alpha}$ are positive integers.

Let $e_{\alpha}, f_{\alpha}$ (for $\alpha$ in the root system of $\mathfrak{g}^{c}$ ) be elements spanning the root spaces corresponding to $\alpha$ and $-\alpha$ respectively. Let $h_{\alpha}$ be a scalar multiple of $\left[e_{\alpha}, f_{\alpha}\right]$ such that $\left[h_{\alpha}, e_{\alpha}\right]=2 e_{\alpha}$. Let $\alpha \in \Delta$. Then by $\mathfrak{g}^{c}(\alpha)$ we denote the subalgebra generated by $e_{\beta}, f_{\beta}$ for $\beta \in \Delta \backslash\{\alpha\}$ along with $e_{\delta}, f_{\delta}$. By $\mathfrak{g}^{c}[\alpha]$ we denote the subalgebra generated by $e_{\beta}, f_{\beta}$ for $\beta \in \Delta \backslash\{\alpha\}$ along with $e_{\alpha}, h_{\alpha}$. Then $\mathfrak{g}^{c}(\alpha)$ is a maximal subalgebra if and only if $n_{\alpha}$ is prime (this statement goes back to BDS49, see also GG78, Chapter 8). The $\mathfrak{g}^{c}(\alpha)$ are semisimple, so they are maximal reductive subalgebras. On the other hand, a maximal reductive, non semisimple subalgebra is contained in a $\mathfrak{g}^{c}[\alpha]$. The latter has a unique (up to conjugation) maximal reductive subalgebra, which we denote by $\mathfrak{g}^{c}[\alpha]^{\prime}$; it is generated by $e_{\beta}, f_{\beta}$ for $\beta \in \Delta \backslash\{\alpha\}$ along with $h_{\alpha}$. However, it is possible that $\mathfrak{g}^{c}[\alpha]^{\prime}$ is contained in a $\mathfrak{g}^{c}(\beta)$. This situation can be characterized as follows. Consider the Dynkin diagram of the set of roots $\Delta \cup\{-\delta\}$ (this is called the extended Dynkin diagram). Now $\mathfrak{g}^{c}[\alpha]^{\prime}$ is contained in a $\mathfrak{g}^{c}(\beta)$ if and only if the diagram obtained by removing the node corresponding to $\alpha$ is not the Dynkin diagram of $\mathfrak{g}^{c}$. (Indeed, if the latter diagram is not the Dynkin diagram of $\mathfrak{g}^{c}$ then $\mathfrak{g}^{c}(\alpha)$ is not equal to $\mathfrak{g}^{c}$ and $\mathfrak{g}^{c}[\alpha]^{\prime} \subset \mathfrak{g}^{c}(\alpha)$. Conversely, if the mentioned diagram is the Dynkin diagram of $\mathfrak{g}^{c}$ then none of the $\mathfrak{g}(\beta)$ contain the semisimple part of $\mathfrak{g}^{c}[\alpha]^{\prime}$.) By inspection this then leads to the maximal reductive $\mathrm{R}$-subalgebras listed in Table 1 . 


\begin{tabular}{|c|c|c|c|}
\hline type & max reductive & type & max reductive \\
\hline$A_{n}$ & $\begin{array}{c}A_{k}+A_{n-1-k}+T_{1} \\
1 \leq k \leq n-1\end{array}$ & $D_{n}$ & $A_{n-1}+T_{1}$ \\
& $D_{n-1}+T_{1}$ \\
\hline$B_{n}$ & $B_{n-1}+T_{1}$ & $E_{6}$ & $D_{5}+T_{1}$ \\
\hline$C_{n}$ & $A_{n-1}+T_{1}$ & $E_{7}$ & $E_{6}+T_{1}$ \\
\hline
\end{tabular}

TABLE 1. Maximal reductive non-semisimple subalgebras of the simple Lie algebras. Here $T_{1}$ denotes a 1-dimensional centre.

Remark 2.1. The subalgebras of Table 1 are also given by Dynkin in Dyn52b, Table 12a. This table lists the regular semisimple subalgebras $\mathfrak{a}^{c} \subset \mathfrak{g}^{c}$ such that no semisimple regular subalgebra $\hat{\mathfrak{a}}^{c}$ exists with $\mathfrak{a}^{c} \subsetneq \hat{\mathfrak{a}}^{c} \subsetneq \mathfrak{g}^{c}$. However, in one case this appears to be not quite correct. In fact, for the embeddings $B_{n-1} \subset B_{n}$ we have the chain

$$
B_{n-1} \subset D_{n} \subset B_{n} .
$$

Here both subalgebras $B_{n-1}$ and $D_{n}$ are regular, but they are not normalized by the same Cartan subalgebra.

We also have the dual chain $D_{n-1} \subset B_{n-1} \subset D_{n}$. But here, obviously, $B_{n-1}$ is not regular in $D_{n}$.

Remark 2.2. Here we point out two oversights that are present in some places in the literature. Firstly, in various works (such as [MP81, Sla81, Yam15]), the subalgebra $A_{1} \oplus A_{1} \oplus A_{1}$ is reported to be maximal in $D_{6}$, while actually it is not. We have that $A_{1} \oplus A_{1} \oplus A_{1}$ is a maximal (non-symmetric) subalgebra in $A_{1} \oplus C_{3}$, which in turn is maximal (and non-symmetric) in $D_{6}$. This has been noted in the tables in LG72.

Secondly, the maximal S-subalgebra $C_{3}$ in $C_{7}$ (giving rise to a non-symmetric embedding) is not listed in some works (such as [MP81, Sla81, Yam15]), whereas it is considered in Dyn52a, LG72, Table VII. All in all, the existence of a maximal $C_{3}$ in $C_{7}$ is a consequence of the anti-self-conjugation (i.e., symplecticity) of the irreducible representation $14^{\prime}=\wedge_{0}^{3} \mathbf{6}$ of $C_{3}$. It is here worth recalling that the action of $C_{3}$ on the $\mathbf{1 4}^{\prime}$ is "of type $E_{7}$ " [Bro69. In [Kac80] it has been proved that such action has a finite number of nilpotent orbits, with one-dimensional ring of invariant polynomials generated by a quartic homogeneous polynomial. The latter is related to the square of the Bekenstein-Hawking entropy of extremal black hole solutions to the "magic" Maxwell-Einstein $\mathcal{N}=2$ supergravity having the split real form $\mathfrak{s p}(6, \mathbb{R})$ of $C_{3}$ as electric-magnetic duality symmetry [GST83. (see BFKM08] for a review and a list of references).

\section{Physical applications of Real embeddings of Reductive Lie ALGEBRAS}

3.1. Super-Ehlers embeddings, and their non-supersymmetric versions. The non-compact real form $\mathfrak{s l}(5, \mathbb{R}) \oplus \mathfrak{s l}(5, \mathbb{R}) \subset E_{8(8)}$ of the maximal (non-symmetric) embedding $A_{4} \oplus A_{4} \subset E_{8}$ is known in supergravity as an example of "super-Ehlers" embedding, concerning the maximally supersymmetric Einstein gravity in 7 spacetime dimensions. Super-Ehlers embeddings, which unify the Ehlers gravity embeddings with the global electric-magnetic duality symmetries of Einstein-Maxwell theories (at least in the cases with symmetric scalar manifolds), have been introduced and studied, in presence of underlying (local) supersymmetry, in [FMT12; in particular, in the Appendices of [FMT12] a general proof of existence of such regular and rank-preserving embeddings, which are non-symmetric in most cases, is given, within an approach completely different from the ones employed in the 
present paper. The general structure of super-Ehlers embeddings is the following (with $3 \leqslant D \leqslant 11$ denoting the number of Lorentzian space-time dimensions):

$$
\mathfrak{g}_{D, \mathcal{N}} \oplus \mathfrak{s l}(D-2, \mathbb{R}) \subset \mathfrak{g}_{3, \mathcal{N}}
$$

where $\mathfrak{g}_{D, \mathcal{N}}$ is the electric-magnetic duality Lie algebra of $D$-dimensional MaxwellEinstein theories endowed with $2 \mathcal{N}$ local supersymmetries (corresponding, in $D=$ 3 , to $\mathcal{N}$-extended supergravity), and $\mathfrak{s l}(D-2, \mathbb{R})$ is the Ehlers symmetry Lie algebra in $D$ Lorentzian dimensions. In presence of supersymmetry, super-Ehlers embeddings are listed and classified in [FMT12] (for the $D=5$ case, see also [FMZ13]). Note that

$$
\begin{aligned}
E_{6(6)} \oplus \mathfrak{s l}(3, \mathbb{R}) & \subset \quad E_{8(8)}, \\
E_{6(-26)} \oplus \mathfrak{s l}(3, \mathbb{R}) & \subset \quad E_{8(-24)}
\end{aligned}
$$

are super-Ehlers embeddings for $\mathcal{N}=16$ and $\mathcal{N}=4$ supergravity theories in $D=5$, respectively. (These two embeddings have been overlooked in Gra72.) On the other hand, non-supersymmetric Maxwell-Einstein theories (coupled to nonlinear sigma model of scalar fields) in various dimensions are not considered in FMT12, but nevertheless they display some "non-supersymmetric Ehlers embeddings"; whose some examples list as follows :

$$
\begin{array}{rccc}
\mathfrak{s o}(6,6) \oplus \mathfrak{s l}(2, \mathbb{R}) & \subset & E_{7(7)} ; \\
\mathfrak{s l}(6, \mathbb{R}) \oplus \mathfrak{s l}(3, \mathbb{R}) & \subset & E_{7(7)} ; \\
\mathfrak{s l}(6, \mathbb{R}) \oplus \mathfrak{s l}(2, \mathbb{R}) & \subset & E_{6(6)} ; \\
\mathfrak{s l}(3, \mathbb{R}) \oplus \mathfrak{s l}(3, \mathbb{R}) \oplus \mathfrak{s l}(3, \mathbb{R}) & \subset & E_{6(6)} .
\end{array}
$$

These embeddings have been discussed in the so-called non-supersymmetric magic Maxwell-Einstein theories in [MPRR17] (see also [MR19]), in which the role of the Ehlers symmetry and related truncations has been highlighted.

3.2. Cubic Jordan algebras, their symmetries and related embeddings. Various embeddings reported in the tables of the present paper have an interpretation in terms of symmetries of cubic Jordan algebras. Such symmetries are given by the $\mathfrak{d e r}$ (derivations), $\mathfrak{s t r}_{0}$ (reduced structure), $\mathfrak{c o n \mathfrak { f }}$ (conformal) and $\mathfrak{q} \mathfrak{c o n f}$ (quasi-conformal) Lie algebras associated to a given cubic Jordan algebra. The quasiconformal realizations of non-compact groups were discovered by the authors of GKN01 and were further developed and applied in GNPW06, GP10, GP09, GNPP08, GNPW07. The conformal groups associated with Jordan algebras were studied much earlier in Gun75, Gun93. Over the reals $\mathbb{R}$, the Lie algebras $\mathfrak{s t r}_{0}, \mathfrak{c o n f}$ and $\mathfrak{q c o n f}$ respectively correspond to the electric-magnetic duality ( $U$-duality 1 ) Lie algebras of some Maxwell-Einstein supergravity theories in $D=5,4,3$ Lorentzian space-time dimensions (cfr. e.g. GP10, and the references therein; in $D=3$ all vectors need to be dualized into scalars); such symmetries are non-linearly realized on the scalars, while vectors do sit in some linear representations of them. Jordan algebras, such as $J_{3}^{\mathbb{H}_{s}}, J_{3}^{\mathbb{C}_{s}}$ and $\mathbb{R} \oplus \boldsymbol{\Gamma}_{m, n}$ with $m$ (or $\left.n\right) \neq 1$ and 5 , are associated to non-supersymmetric models MPRR17, MR19. When considering simple cubic Jordan algebras, all the aforementioned related Lie algebras fill the first $(\mathfrak{d e r})$, second $\left(\mathfrak{s t r}_{0}\right)$, third ( $\left.\mathfrak{c o n f}\right)$ and fourth $(\mathfrak{q} \mathfrak{c o n f})$ rows of the relevant Magic Square of Freudenthal-Rozenfeld-Tits [Fre63, Tit66, Roz56], and the following maximal

\footnotetext{
${ }^{1}$ Here $U$-duality is referred to as the "continuous" symmetries of CJ79 CJ78. Their discrete versions are the $U$-duality non-perturbative string theory symmetries introduced in [HT95].
} 
embeddings hold (see CCM15, and references therein):

$$
\begin{aligned}
& \mathfrak{d e r} \subset \mathfrak{s t r}_{0} ; \\
& \mathfrak{d e r} \oplus \mathfrak{s l}(2, \mathbb{R}) \quad \subset \quad \mathfrak{c o n f} ; \\
& \mathfrak{s t r}_{0} \oplus \mathfrak{s o}(1,1) \quad \subset \quad \mathfrak{c o n f} ; \\
& \mathfrak{c o n f} \oplus \mathfrak{s l}(2, \mathbb{R}) \quad \subset \quad \mathfrak{q c o n f} ; \\
& \mathfrak{s t r}_{0} \oplus \mathfrak{s l}(3, \mathbb{R}) \quad \subset \quad \mathfrak{q c o n f} ; \\
& \mathfrak{d e r} \oplus G_{2(2)} \subset \mathbf{q c o n f} .
\end{aligned}
$$

Within the physical interpretation of such symmetries as $U$-duality Lie algebras,

\begin{tabular}{|c|c|c|c|c|}
\hline & $\mathbb{R}$ & $\mathbb{C}$ & $\mathbb{H}$ & $\mathbb{O}$ \\
\hline $\mathbb{R}$ & $S O(3)$ & $S U(3)$ & $U S p(3)$ & $F_{4(-52)}$ \\
\hline $\mathbb{C}_{s}$ & $S L(3, \mathbb{R})$ & $S L(3, \mathbb{C})$ & $S L(3, \mathbb{H})$ & $E_{6(-26)}$ \\
\hline $\mathbb{H}_{s}$ & $S p(6, \mathbb{R})$ & $S U(3,3)$ & $S O^{*}(12)$ & $E_{7(-25)}$ \\
\hline $\mathbb{O}_{s}$ & $F_{4(4)}$ & $E_{6(2)}$ & $E_{7(-5)}$ & $E_{8(-24)}$ \\
\hline
\end{tabular}

TABLE 2. The single-split MS $\mathcal{L}_{3}\left(\mathbb{A}_{s}, \mathbb{B}\right)$ [GST83. (see [CCM15] for details)

\begin{tabular}{|c|c|c|c|c|}
\hline & $\mathbb{R}$ & $\mathbb{C}_{s}$ & $\mathbb{H}_{s}$ & $\mathbb{O}_{s}$ \\
\hline $\mathbb{R}$ & $S O(3)$ & $S L(3, \mathbb{R})$ & $S p(3, \mathbb{R})$ & $F_{4(4)}$ \\
\hline $\mathbb{C}_{s}$ & $S L(3, \mathbb{R})$ & $S L(3, \mathbb{R}) \times S L(3, \mathbb{R})$ & $S L(6, \mathbb{R})$ & $E_{6(6)}$ \\
\hline $\mathbb{H}_{s}$ & $S p(6, \mathbb{R})$ & $S L(6, \mathbb{R})$ & $S O(6,6)$ & $E_{7(7)}$ \\
\hline $\mathbb{O}_{s}$ & $F_{4(4)}$ & $E_{6(6)}$ & $E_{7(7)}$ & $E_{8(8)}$ \\
\hline
\end{tabular}

TABLE 3. The double-split MS $\mathcal{L}_{3}\left(\mathbb{A}_{s}, \mathbb{B}_{s}\right)$ [BS03] (see [CCM15] for further details)

the commuting $\mathfrak{s o}(1,1)$ in (3.8) can be regarded as the Kaluza-Klein compactification radius of the $S^{1}$-reduction $D=5 \rightarrow 4$; alternatively, such an $\mathfrak{s o}(1,1)$ can also be conceived as the Lie algebra of the pseudo-Kähler connection of the pseudospecial Kähler (and pseudo-Riemannian) symmetric coset2 $\frac{C o n f}{S r_{0} \otimes S O(1,1)}$, obtained from $\frac{S t r_{0}}{m c s\left(\text { Str }_{0}\right)}$ by applying the inverse $R^{*}$-map pertaining to a timelike compactification $D=s+t=4+1 \rightarrow 4+0$ where $s$ and $t$ respectively denote the number of spacelike and timelike dimensions dWVVP93, AC09, $\mathrm{CLL}^{+98}$. On the other hand, the commuting $\mathfrak{s l}(2, \mathbb{R})$ in (3.9) can be identified with the Ehlers symmetry arising from the $S^{1}$-reduction $D=4 \rightarrow 3$; such an $\mathfrak{s l}(2, \mathbb{R})$ can also be interpreted as the connection of the para-quaternionic (and pseudo-Riemannian) symmetric coset $\frac{Q \operatorname{Conf}}{\operatorname{Conf} \otimes S L(2, \mathbb{R})}$, obtained from $\frac{\operatorname{Conf}}{m c s(\operatorname{Conf})}$ by applying the inverse $c^{*}$-map pertaining to a timelike compactification $D=(3,1) \rightarrow(3,0)$ BMG88, CFG89, CLL $^{+} 98$. As the embeddings (3.6)-(3.11) are obtained by moving along the columns of the relevant (real form of the) Magic Square (for a fixed row entry), another class of embeddings can be obtained by moving along the rows of the relevant Magic Square (for a fixed column entry). In the symmetric (real forms of the) rank-3 Magic Square, as the double-split $\mathcal{L}_{3}\left(\mathbb{A}_{s}, \mathbb{B}_{s}\right)$ given in Table 3 , these embeddings trivially coincide with (3.6)-(3.11), but their intepretation corresponds to the restriction from one (division $\mathbb{A}$ or split $\mathbb{A}_{s}$ ) algebra to a smaller one. For the non-symmetric, single-split Magic

\footnotetext{
${ }^{2}$ As intuitive, the names starting with a capital letter denote the Lie groups whose Lie algebra is the same name starting lowercase. Moreover, " $m c s$ " denotes the maximal compact subgroup.
} 
Square $\mathcal{L}_{3}\left(\mathbb{A}_{s}, \mathbb{B}\right)$ reported in Table 2 different maximal embeddings hold true, which are different from the ones given in (3.6)-(3.11); namely $\left(G_{2}^{\mathrm{cpt}} \equiv G_{2(-14)}\right)$ :

$$
\begin{aligned}
\mathfrak{d}\left(J_{3}^{\mathbb{R}}\right) & \subset \mathfrak{d}\left(J_{3}^{\mathbb{C}}\right) \\
\mathfrak{s u}(2) \oplus \mathfrak{d}\left(J_{3}^{\mathbb{R}}\right) & \subset \mathfrak{d}\left(J_{3}^{\mathbb{H}}\right) \\
\mathfrak{u}(1) \oplus \mathfrak{d}\left(J_{3}^{\mathbb{C}}\right) & \subset \mathfrak{d}\left(J_{3}^{\mathbb{H}}\right) \\
\mathfrak{s u}(2) \oplus \mathfrak{d}\left(J_{3}^{\mathbb{H}}\right) & \subset \mathfrak{d}\left(J_{3}^{\mathbb{D}}\right) \\
\mathfrak{s u}(3) \oplus \mathfrak{d}\left(J_{3}^{\mathbb{C}}\right) & \subset \mathfrak{d}\left(J_{3}^{\mathbb{Q}}\right) \\
G_{2}^{\mathrm{cpt}} \oplus \mathfrak{d}\left(J_{3}^{\mathbb{R}}\right) & \subset \mathfrak{d}\left(J_{3}^{\mathbb{H}}\right)
\end{aligned}
$$

(where $\mathfrak{d}$ can be each of $\mathfrak{d e r}, \mathfrak{s t r} \mathfrak{r}_{0}, \mathfrak{c o n} \mathfrak{f}, \mathfrak{q c o n f}$ ).

Similar embeddings holds for simple Lorentzian cubic Jordan algebras (see [CCM15]).

3.3. Semisimple subalgebras of simple Jordan algebras, and their symmetries. Another remarkable class of embedding stems from the relation between simple cubic Jordan algebras [JvNW34] $J_{3}^{\mathbb{A}}$ or $J_{3}^{\mathbb{A}_{s}}$ and some elements of the (biparametric) infinite sequence of semi-simple Jordan algebras $\mathbb{R} \oplus \boldsymbol{\Gamma}_{m, n}$ mentioned above, exploiting the Jordan-algebraic isomorphisms $J_{2}^{\mathbb{A}} \cong \boldsymbol{\Gamma}_{1, q+1}\left(\cong \boldsymbol{\Gamma}_{q+1,1}\right)$ and $J_{2}^{\mathbb{A}_{s}} \cong \boldsymbol{\Gamma}_{q+2+1, q / 2+1}$, where $q:=\operatorname{dim}_{R} \mathbb{A}=8,4,2,1$ for $\mathbb{A}=\mathbb{O}, \mathbb{H}, \mathbb{C}, \mathbb{R}$, and $q:=\operatorname{dim}_{R} \mathbb{A}_{s}=$ $8,4,2$ for $\mathbb{A}_{s}=\mathbb{O}_{s}, \mathbb{H}_{s}, \mathbb{C}_{s}$ (see e.g. App. A of [GP10] - and Refs. therein - for an introduction to division and split algebras). Indeed, the following (maximal, rank-preserving) Jordan-algebraic embeddings hold :

$$
\begin{aligned}
& J_{3}^{\mathbb{A}} \supset \mathbb{R} \oplus J_{2}^{\mathbb{A}} \cong \mathbb{R} \oplus \boldsymbol{\Gamma}_{1, q+1} ; \\
& J_{3}^{\mathbb{A}_{s}} \supset \mathbb{R} \oplus J_{2}^{\mathbb{A}_{s}} \cong \mathbb{R} \oplus \boldsymbol{\Gamma}_{q / 2+1, q / 2+1} .
\end{aligned}
$$

Thus, one can consider their consequences at the level of symmetries of cubic Jordan algebras defined over the corresponding algebras, obtaining :

$$
\begin{aligned}
\mathfrak{d}\left(\mathbb{R} \oplus J_{2}^{\mathbb{A}}\right) \oplus \mathcal{A}_{q} & \subset \mathfrak{d}\left(J_{3}^{\mathbb{A}}\right) \\
\mathfrak{d}\left(\mathbb{R} \oplus J_{2}^{\mathbb{A}_{s}}\right) \oplus \widetilde{\mathcal{A}}_{q} & \subset \mathfrak{d}\left(J_{3}^{\mathbb{A}_{s}}\right)
\end{aligned}
$$

(where as above $\mathfrak{d}$ can be each of $\mathfrak{d e r}, \mathfrak{s t r} \mathfrak{r}_{0}, \mathfrak{c o n} \mathfrak{f}, \mathfrak{q c o n \mathfrak { f }}$ ).

Note the maximal nature of the embeddings (3.20)-3.21), as well as the presence of the commuting algebras $\mathcal{A}_{q}$ and $\widetilde{\mathcal{A}}_{q}$, defined as follows:

$$
\begin{aligned}
& \mathcal{A}_{q}:=\operatorname{tri}(q) \ominus s o(q)=\varnothing, \mathfrak{s o}(3), \mathfrak{s o}(2), \varnothing \text { for } q=8,4,2,1 ; \\
& \widetilde{\mathcal{A}}_{q}:=\widetilde{\mathfrak{t r i}}(q) \ominus \widetilde{s o}(q)=\varnothing, \mathfrak{s l}(2, \mathbb{R}), \mathfrak{s o}(1,1) \text { for } q=8,4,2,
\end{aligned}
$$

where $\mathfrak{t r i}$ and $\mathfrak{s o}$ respectively denote the triality and orthogonal (norm-preserving) symmetries (and they are tilded when pertaining to split algebras; see e.g. CFMZ10, CCM13, and Refs. therein). Analogous results hold for Lorentzian Jordan algebras. Within the physical (U-duality) interpretation, $\mathcal{A}_{q}$ and $\widetilde{\mathcal{A}}_{q}$ are consistent with the properties of spinors in $q+2$ dimensions, with Lorentzian signature $(t, s)=(1, q+1)$ respectively Kleinian signature $(t, s)=(q / 2+1, q / 2+1)$; indeed, the electric-magnetic ( $U$-duality) symmetry Lie algebra in $D=6$ (Lorentzian) space-time dimensions is $\mathfrak{s o}(1, q+1) \oplus \mathcal{A}_{q}$ for $\mathbb{A}$-based theories (which are endowed with minimal, chiral $(1,0)$ supersymmetry) and $\mathfrak{s o}(q / 2+1, q / 2+1) \oplus \widetilde{\mathcal{A}}_{q}$ for $\mathbb{A}_{s^{-}}$ based theories (which are non-supersymmetric for $q=2,4$, and endowed with maximal, non-chiral $(2,2)$ supersymmetry for $q=8)$; cfr. e.g. KT83, GSS11 (and Refs. therein) and [MNY11] for further discussion. 


\section{Computational methods}

In this section we briefly describe the computational methods that we used to construct the real forms of the maximal semisimple (or reductive) subalgebras of the simple Lie algebras of ranks up to 8 . We have one procedure for constructing the regular subalgebras (taken from [DFdG15]) and one procedure for the S-subalgebras (from [FdG15]). First we recall some general notation, and subsequently describe the methods in two subsections. Our main reference for the general theory is Oni04.

By $\iota \in \mathbb{C}$ we denote the imaginary unit. Let $\mathfrak{g}^{c}$ be a complex simple Lie algebra. An anti-involution of $\mathfrak{g}^{c}$ is a map $\eta: \mathfrak{g}^{c} \rightarrow \mathfrak{g}^{c}$ with $\eta(x+y)=\eta(x)+\eta(y), \eta(\mu x)=\bar{\mu} x$, $\eta([x, y])=[\eta(x), \eta(y)], \eta(\eta(x))=x$ for all $x, y \in \mathfrak{g}^{c}, \mu \in \mathbb{C}$. If $\eta: \mathfrak{g}^{c} \rightarrow \mathfrak{g}^{c}$ is any map then we set $\mathfrak{g}_{\eta}^{c}=\left\{x \in \mathfrak{g}^{c} \mid \eta(x)=x\right\}$.

A real form $\mathfrak{g}$ of $\mathfrak{g}^{c}$ is given by three maps, $\tau, \sigma, \theta: \mathfrak{g}^{c} \rightarrow \mathfrak{g}^{c}$ where

- $\tau, \sigma$ are anti-involutions and $\theta$ is an involution,

- $\theta=\tau \sigma=\sigma \tau$,

- $\mathfrak{g}_{\tau}^{c}$ is compact and $\mathfrak{g}_{\sigma}^{c}=\mathfrak{g}$,

- setting $\mathfrak{k}=\{x \in \mathfrak{g} \mid \theta(x)=x\}, \mathfrak{p}=\{x \in \mathfrak{g} \mid \theta(x)=-x\}$; then $\mathfrak{g}=\mathfrak{k} \oplus \mathfrak{p}$ is a Cartan decomposition of $\mathfrak{g}$ (note that $\theta(\mathfrak{g})=\mathfrak{g}$ because $\theta$ commutes with $\sigma)$; the restriction of $\theta$ to $\mathfrak{g}$ is called a Cartan involution of $\mathfrak{g}$,

- set $\mathfrak{u}=\mathfrak{g}_{\tau}^{c}$, then $\theta$ leaves $\mathfrak{u}$ invariant so that $\mathfrak{u}=\mathfrak{u}_{1} \oplus \mathfrak{u}_{-1}$ (the eigenspaces of $\theta$ with eigenvalues \pm 1 ) and $\mathfrak{k}=\mathfrak{u}_{1}, \mathfrak{p}=\iota \mathfrak{u}_{-1}$.

Let $\mathfrak{a} \subset \mathfrak{g}$ be a semisimple subalgebra and $\mathfrak{a}^{c}=\mathfrak{a}+\iota \mathfrak{a}$, which is a semisimple subalgebra of $\mathfrak{g}^{c}$. Then $\mathfrak{a}$ is a real form of the complex subalgebra $\mathfrak{a}^{c}$. So $\mathfrak{a}$ has a Cartan decomposition $\mathfrak{a}=\mathfrak{k}_{\mathfrak{a}} \oplus \mathfrak{p}_{\mathfrak{a}}$. It follows from the Karpelevich-Mostow theorem (Oni04, Corollary 1 of $\S 6$ ) that $\mathfrak{a}$ is conjugate by an inner automorphism to a subalgebra $\mathfrak{a}^{\prime}$ such that $\mathfrak{k}_{\mathfrak{a}^{\prime}} \subset \mathfrak{k}$ and $\mathfrak{p}_{\mathfrak{a}^{\prime}} \subset \mathfrak{p}$. Equivalently, $\theta$ maps $\mathfrak{a}^{\prime}$ to itself, and its restriction to $\mathfrak{a}^{\prime}$ is a Cartan involution of $\mathfrak{a}^{\prime}$. So we may restrict to finding $\theta$-stable subalgebras of $\mathfrak{g}$.

4.1. Real forms of regular subalgebras. We say that a subalgebra $\mathfrak{a}$ of $\mathfrak{g}$ is regular if it is normalized by a Cartan subalgebra $\mathfrak{h}$ of $\mathfrak{g}$. If we want to stress the particular Cartan subalgebra that we are referring to we say that $\mathfrak{a}$ is $\mathfrak{h}$-regular. In that case $\mathfrak{a}^{c}$ is spanned by root spaces of $\mathfrak{g}^{c}$ (relative to $\mathfrak{h}^{c}$ ) along with $\mathfrak{a}^{c} \cap \mathfrak{h}^{c}$. By $\Psi\left(\mathfrak{h}^{c}, \mathfrak{a}^{c}\right)$ we denote the set of roots involved in this. It is a subset of the root system of $\mathfrak{g}^{c}$ with respect to $\mathfrak{h}^{c}$.

Let $G^{c}$ denote the adjoint group of $\mathfrak{g}^{c}$. This group can be characterized in several ways. Firstly it is the connected algebraic subgroup of $\operatorname{GL}\left(\mathfrak{g}^{c}\right)$ with Lie algebra $\operatorname{adg}^{c}$. Secondly it is the group generated by all elements $\exp (a d x)$ where $x \in \mathfrak{g}^{c}$ is nilpotent. Thirdly, it is the identity component of $\operatorname{Aut}\left(\mathfrak{g}^{c}\right)$. By $G$ we denote the group consisting of all $g \in G^{c}$ such that $g(\mathfrak{g})=\mathfrak{g}$. If we represent elements of $G^{c}$ by their matrices with respect to a basis of $\mathfrak{g}$ then $G=G^{c}(\mathbb{R})$, the group of all elements of $G^{c}$ with coefficients in $\mathbb{R}$. Let $G_{0}$ be the identity component of $G$. In this section we outline how to obtain the regular semisimple subalgebras of $\mathfrak{g}$ up to conjugacy by $G_{0}$.

First of all, Dynkin $([\overline{D y n} 52 \mathrm{~b})$ devised an algorithm to obtain the regular semisimple subalgebras of $\mathfrak{g}^{c}$ up to conjugacy by $G^{c}$ (see [Gra17, §5.9 for a recent account). We can use this algorithm to obtain all $\mathfrak{h}^{c}$-regular semisimple subalgebras up to $G^{c}$-conjugacy, for a fixed Cartan subalgebra $\mathfrak{h}^{c}$ of $\mathfrak{g}^{c}$. We have that two $\mathfrak{h}^{c}$-regular semisimple subalgebras $\mathfrak{a}_{1}^{c}, \mathfrak{a}_{2}^{c}$ of $\mathfrak{g}^{c}$ are $G^{c}$-conjugate if and only if $\Psi\left(\mathfrak{h}^{c}, \mathfrak{a}_{1}^{c}\right), \Psi\left(\mathfrak{h}^{c}, \mathfrak{a}_{2}^{c}\right)$ are $W\left(\mathfrak{g}^{c}, \mathfrak{h}^{c}\right)$-conjugate, where the latter denotes the Weyl group of the root system of $\mathfrak{g}^{c}$ with respect to $\mathfrak{h}^{c}$. 
If one is only interested in subalgebras of $\mathfrak{g}^{c}$ then it suffices to consider just one Cartan subalgebra as they are all conjugate under $G^{c}$. In general the real form $\mathfrak{g}$ has more Cartan subalgebras that are non-conjugate. Sugiura ([Sug59]) proved that $\mathfrak{g}$ has a finite number of Cartan subalgebras up to $G_{0}$-conjugacy. In DFG13 Sugiura's method was made into an algorithm for listing the Cartan subalgebras of $\mathfrak{g}$ up to conjugacy by $G_{0}$.

Let $\mathfrak{a}$ be a regular semisimple subalgebra of $\mathfrak{g}$. Then the normalizer $\mathfrak{n}_{\mathfrak{g}}(\mathfrak{a})=\{x \in$ $\mathfrak{g} \mid[x, \mathfrak{a}] \subset \mathfrak{a}\}$ is reductive. Therefore it has a unique maximally noncompact Cartan subalgebra (that is, a Cartan subalgebra whose intersection with $\mathfrak{p}$ has maximal dimension, see Kna02, Proposition 6.61). We say that $\mathfrak{a}$ is strongly $\mathfrak{h}$-regular if $\mathfrak{h}$ is a maximally noncompact Cartan subalgebra of $\mathfrak{n}_{\mathfrak{g}}(\mathfrak{a})$.

Let $\mathfrak{h}$ be a Cartan subalgebra of $\mathfrak{g}$. Set $N=\left\{g \in G_{0} \mid g(\mathfrak{h})=\mathfrak{h}\right\}$ and $Z=\{g \in$ $G_{0} \mid g(x)=x$ for all $\left.x \in \mathfrak{h}\right\}$. Then $W(\mathfrak{g}, \mathfrak{h})=N / Z$ is called the real Weyl group of $\mathfrak{h}$. It can naturally be identified with a subgroup of $W\left(\mathfrak{g}^{c}, \mathfrak{h}^{c}\right)$, (see [Kna02, $\S$ VII.8). There are algorithms to compute $W(\mathfrak{g}, \mathfrak{h})$, see DG19.

We have the following criterion: Let $\mathfrak{a}_{1}, \mathfrak{a}_{2}$ be two strongly $\mathfrak{h}$-regular semisimple subalgebras of $\mathfrak{g}$. They are $G_{0}$-conjugate if and only if $\Psi\left(\mathfrak{h}^{c}, \mathfrak{a}_{1}^{c}\right), \Psi\left(\mathfrak{h}^{c}, \mathfrak{a}_{2}^{c}\right)$ are conjugate under $W(\mathfrak{g}, \mathfrak{h})$ (see DFdG15], Proposition 24). This gives an immediate method for deciding whether $\mathfrak{a}_{1}, \mathfrak{a}_{2}$ are $G_{0}$-conjugate.

Now the algorithm for obtaining all regular semisimple subalgebras of $\mathfrak{g}$ (up to $G_{0}$-conjugacy) runs as follows:

(1) Compute the Cartan subalgebras of $\mathfrak{g}$ (up to $G_{0}$-conjugacy). For each obtained Cartan subalgebra $\mathfrak{h}$ perform the following steps:

(a) Compute the $\mathfrak{h}^{c}$-regular subalgebras of $\mathfrak{g}^{c}$ using Dynkin's algorithm. For each obtained subalgebra $\mathfrak{a}^{c}$ perform the following steps:

(i) Compute the stabilizer $S$ of $\Psi\left(\mathfrak{h}^{c}, \mathfrak{a}^{c}\right)$ in $W=W\left(\mathfrak{g}^{c}, \mathfrak{h}^{c}\right)$. Compute a list of representatives $w_{1}, \ldots, w_{s}$ of the double cosets $W(\mathfrak{g}, \mathfrak{h}) w_{i} S$ in $W$.

(ii) Construct the $\mathfrak{h}^{c}$-regular subalgebras $\mathfrak{a}_{i}^{c}$ of $\mathfrak{g}^{c}$ with $\Psi\left(\mathfrak{h}^{c}, \mathfrak{a}_{i}^{c}\right)=$ $w_{i} \cdot \Psi\left(\mathfrak{h}^{c}, \mathfrak{a}^{c}\right)$.

(iii) Throw away the $\mathfrak{a}_{i}^{c}$ that are not $\sigma$-stable.

(iv) Of the remaining ones compute a basis of $\mathfrak{a}_{i}=\mathfrak{a}_{i}^{c} \cap \mathfrak{g}$, and throw away those that are not strongly $\mathfrak{h}$-regular. Add the remaining ones to the final list.

As before, let $\theta$ denote the Cartan involution of $\mathfrak{g}$. The Cartan subalgebras found in the first step are $\theta$-stable (see the algorithm in [DFG13]). Therefore the subalgebras constructed by the above procedure are automatically $\theta$-stable as well ([DFdG15], Proposition 21).

Note that the above algorithm is correct. Indeed, each $\mathfrak{h}^{c}$-regular semisimple subalgebra $\mathfrak{b}^{c}$ of $\mathfrak{g}^{c}$ that is $G^{c}$-conjugate to $\mathfrak{a}^{c}$ has $\Psi\left(\mathfrak{h}^{c}, \mathfrak{b}^{c}\right)=w \cdot \Psi\left(\mathfrak{h}^{c}, \mathfrak{a}^{c}\right)$ for some $\mathfrak{a}^{c}$ from the initial list. Furthermore, note that the $w_{i} \cdot \Psi\left(\mathfrak{h}^{c}, \mathfrak{a}^{c}\right)$ exhaust the images under $W\left(\mathfrak{g}^{c}, \mathfrak{h}^{c}\right)$ of $\Psi\left(\mathfrak{h}^{c}, \mathfrak{a}^{c}\right)$ up to conjugacy by $W(\mathfrak{g}, \mathfrak{h})$.

4.2. Real forms of S-subalgebras. From Section 2 we recall that a subalgebra of $\mathfrak{g}^{c}$ is called an $S$-subalgebra if it is not contained in a proper regular subalgebra. For these subalgebras we have no method to list all real forms in $\mathfrak{g}$ up to conjugacy by $G_{0}$. Therefore we are more modest and consider the following question. Let $\varepsilon: \mathfrak{g}^{c} \hookrightarrow \tilde{\mathfrak{g}}^{c}$ be an embedding of semisimple complex Lie algebras and let $\mathfrak{g}$ be a real form of $\mathfrak{g}^{c}$. Find (up to isomorphism) the real forms $\tilde{\mathfrak{g}}$ of $\tilde{\mathfrak{g}}^{c}$ such that $\varepsilon(\mathfrak{g}) \subset \tilde{\mathfrak{g}}$. Because two real forms $\tilde{\mathfrak{g}}, \tilde{\mathfrak{g}}^{\prime}$ of $\tilde{\mathfrak{g}}^{c}$ are isomorphic if and only if there is a $\phi \in \operatorname{Aut}\left(\tilde{\mathfrak{g}}^{c}\right)$ with $\phi(\tilde{\mathfrak{g}})=\tilde{\mathfrak{g}}^{\prime}($ Oni04,,$\S 2$, Proposition 1) we may replace the given embedding $\varepsilon$ by $\phi \circ \varepsilon$ for any $\phi \in \operatorname{Aut}\left(\tilde{\mathfrak{g}}^{c}\right)$. 
Let $\theta, \tilde{\theta}$ be Cartan involutions of $\mathfrak{g}, \tilde{\mathfrak{g}}$ respectively. A classical theorem of Karpelevich states that $\varepsilon(\mathfrak{g}) \subset \tilde{\mathfrak{g}}$ if and only if $\varepsilon \circ \theta=\tilde{\theta} \circ \varepsilon$ (see OV94, Theorem 3.7 of Chapter 6 ). Here we consider $\theta$ and $\mathfrak{g}$ to be given, and hence have to construct real forms $\tilde{\mathfrak{g}}$ with Cartan involution $\tilde{\theta}$ satisfying the mentioned condition. Our method is based on Proposition 4.1 .

Let $\sigma: \mathfrak{g}^{c} \rightarrow \mathfrak{g}^{c}$ be the conjugation with respect to the real form $\mathfrak{g}$. Let $\mathfrak{u}$ be a fixed compact form of $\mathfrak{g}^{c}$ with corresponding conjugation $\tau: \mathfrak{g}^{c} \rightarrow \mathfrak{g}^{c}$ with $\sigma \tau=\tau \sigma$. Set $\theta=\sigma \tau$. Then the restriction of $\theta$ to $\mathfrak{g}$ is a Cartan involution of $\mathfrak{g}$. From [FdG15] we have the following result.

Proposition 4.1. Let $\tilde{\mathfrak{g}} \subset \tilde{\mathfrak{g}}^{c}$ be a real form of $\tilde{\mathfrak{g}}^{c}$ such that $\varepsilon(\mathfrak{g}) \subset \tilde{\mathfrak{g}}$. Then there are a compact form $\tilde{\mathfrak{u}} \subset \tilde{\mathfrak{g}}^{c}$ of $\tilde{\mathfrak{g}}^{c}$, with conjugation $\tilde{\tau}: \tilde{\mathfrak{g}}^{c} \rightarrow \tilde{\mathfrak{g}}^{c}$, and an involution $\tilde{\theta}$ of $\tilde{\mathfrak{g}}^{c}$ such that

(1) $\varepsilon(\mathfrak{u}) \subset \tilde{\mathfrak{u}}$,

(2) $\varepsilon \theta=\tilde{\theta} \varepsilon$,

(3) $\tilde{\theta} \tilde{\tau}=\tilde{\tau} \tilde{\theta}$,

(4) there is a Cartan decomposition $\tilde{\mathfrak{g}}=\tilde{\mathfrak{k}} \oplus \tilde{\mathfrak{p}}$, such that the restriction of $\tilde{\theta}$ to $\tilde{\mathfrak{g}}$ is the corresponding Cartan involution, and $\tilde{\mathfrak{u}}=\tilde{\mathfrak{k}} \oplus$ î̉.

Conversely, if $\tilde{\mathfrak{u}} \subset \tilde{\mathfrak{g}}$ is a compact form, with corresponding conjugation $\tilde{\tau}$, and $\tilde{\theta}$ is an involution of $\tilde{\mathfrak{g}}^{c}$ such that (1), (2) and (3) hold, then $\tilde{\theta}$ leaves $\tilde{\mathfrak{u}}$ invariant, and setting $\tilde{\mathfrak{k}}=\tilde{\mathfrak{u}}_{1}, \tilde{\mathfrak{p}}=\imath \tilde{\mathfrak{u}}_{-1}$ (where $\tilde{\mathfrak{u}}_{k}$ is the $k$-eigenspace of $\left.\tilde{\theta}\right)$, we get that $\tilde{\mathfrak{g}}=\tilde{\mathfrak{k}} \oplus \tilde{\mathfrak{p}}$ is a real form of $\tilde{\mathfrak{g}}^{c}$ with $\varepsilon(\mathfrak{g}) \subset \tilde{\mathfrak{g}}$.

To construct the real forms $\tilde{\mathfrak{g}}$ the strategy is now the following. First we fix a compact form $\tilde{\mathfrak{u}}$ of $\tilde{\mathfrak{g}}^{c}$ and replace $\varepsilon$ by $\phi \varepsilon$ for a $\phi \in \operatorname{Aut}\left(\tilde{\mathfrak{g}}^{c}\right)$ in order to have $\varepsilon(\mathfrak{u}) \subset \tilde{\mathfrak{u}}$. Secondly we construct the space

$$
\mathcal{A}=\left\{A \in \operatorname{End}\left(\tilde{\mathfrak{g}}^{c}\right) \mid A(\operatorname{ad}(\varepsilon \theta(y)))=(\operatorname{ad} \varepsilon(y)) A \text { for all } y \in \mathfrak{g}^{c}\right\} .
$$

(Note that a basis of $\mathcal{A}$ can be computed by solving a set of linear equations; FdG15] also has a more efficient method to construct $\mathcal{A}$.) Let $\tilde{\theta}$ be an involution of $\tilde{\mathfrak{g}}^{c}$. Then $\varepsilon \theta=\tilde{\theta} \varepsilon$ if and only if $\tilde{\theta} \in \mathcal{A}$ and

$$
\tilde{\theta}(\operatorname{ad} x) \tilde{\theta}=\operatorname{ad} \tilde{\theta}(x) \text { for all } x \in \tilde{\mathfrak{g}}^{c}
$$

([FdG15], Proposition 3.6). Hence $\mathcal{A}$ contains the maps $\tilde{\theta}$. The conditions that $\tilde{\theta}$ be an involution, (4.1), (3) are translated to polynomial equations on the coefficients of $\tilde{\theta}$ with respect to a basis of $\mathcal{A}$. These polynomial equations are then studied and solved by means of the technique of Gröbner bases (see [CLO15]).

For the details of this procedure we refer to FdG15]. But we do remark that if $\varepsilon(\mathfrak{g}) \subset \tilde{\mathfrak{g}}$ for some real form $\tilde{\mathfrak{g}}$ of $\tilde{\mathfrak{g}}^{c}$ then we get just one subalgebra of $\tilde{\mathfrak{g}}$. However, there may be more subalgebras $\mathfrak{a} \subset \tilde{\mathfrak{g}}$ such that $\mathfrak{a}^{c}$ is $G^{c}$-conjugate to $\varepsilon\left(\mathfrak{g}^{c}\right)$, without $\mathfrak{a}$ being $G_{0}$-conjugate to $\varepsilon(\mathfrak{g})$. This method does not detect such a situation (unlike the method for the regular case). Furthermore, there are cases in type $D_{n}$ for $n$ even, where there are subalgebras $\mathfrak{a}_{1}^{c}, \mathfrak{a}_{2}^{c} \subset \tilde{\mathfrak{g}}^{c}$ that are not conjugate under $G^{c}$, but are conjugate under an outer automorphism. In these cases we just consider one of the corresponding embeddings in $\tilde{\mathfrak{g}}^{c}$ because changing the embedding $\varepsilon$ to a $\phi \varepsilon$ may identify the subalgebras $\mathfrak{a}_{1}^{c}, \mathfrak{a}_{2}^{c}$.

4.3. Implementation. We have implemented these methods in the computer algebra system GAP4 (GAP18) using the packages SLA ( dGGT19]) and CoReLG ([DFdG19]). The implementation of the algorithm of Section 4.1] is quite straightforward. For the procedure indicated in Section 4.2 we remark that the package SLA contains tables with the semisimple subalgebras of the simple complex Lie algebras. The embeddings $\varepsilon$ are simply given by those tables. 


\section{THE TABLES}

Here we give the tables that we have obtained. In these we use the notation of real forms as used in Kna02. Several authors use different notation, for instance, $\mathfrak{s u}^{*}(2 n)$ instead of $\mathfrak{s l}(4, \mathbb{H})$. Moreover, we use the mathematical notation for symplectic algebras : $C_{n}$ corresponds to $\mathfrak{s p}_{n}$, and not to $\mathfrak{s p}_{2 n}$, as in a large part of the physics literature. We also denote by $G_{2}^{\mathrm{cpt}}, F_{4}^{\mathrm{cpt}}, E_{6}^{\mathrm{cpt}}$ and $E_{7}^{\mathrm{cpt}}$ the compact real forms of $G_{2}, F_{4}, E_{6}$ and $E_{7}$, respectively. In the literature, they are also indicated by $G_{2(-14)}, F_{4(-52)}, E_{6(-78)}$ and $E_{7(-133)}$.

As written in the previous section, the algorithm for the regular subalgebras determines them up to conjugacy by the adjoint group. Therefore, occasionally it happens that we get more than one real embedding of the same type. This is indicated in the tables by putting the number of those embeddings (e.g., as $2 \times$ ). For the S-subalgebras we can only determine whether there is an embedding, except when the S-subalgebra is of type $A_{1}$. In those cases the subalgebras correspond to the nilpotent orbits in $\mathfrak{g}$ (see [CM93]); so for those subalgebras we also know how many there are up to conjugacy by the adjoint group. It may also happen that there is more than one S-subalgebra of type $A_{1}$. In those cases the different occurrences of these subalgebras are distinguished by an upper index, giving the Dynkin index of the subalgebra.

The S-subalgebras of the real forms of the exceptional algebras have also been determined by Komrakov Kom90. However, his table for $E_{6}$ has quite a lot of omissions (for example, the real forms $E_{6(6)}, E_{6(-26)}$ appear to have been forgotten altogether). Also the table for the S-subalgebras of the real forms of type $E_{6}$ in FdG15] have a number of errors and omissions (some subalgebras have been identified incorrectly, others have been omitted).

Type $A_{2}$

Table 4: Maximal subalgebras of $\mathfrak{s u}(1,2)$.

\begin{tabular}{|l|c|c|}
\hline type & real subalgebras & \\
\hline$A_{1}$ & $\begin{array}{c}\mathfrak{s l}(2, \mathbb{R}) \oplus \mathfrak{u}(1) \\
\mathfrak{s u}(2) \oplus \mathfrak{u}(1)\end{array}$ & $\mathrm{R}$ \\
\hline$A_{1}$ & $\mathfrak{s l}(2, \mathbb{R})$ & $\mathrm{S}$ \\
\hline
\end{tabular}

Table 5: Maximal subalgebras of $\mathfrak{s l}(3, \mathbb{R})$.

\begin{tabular}{|l|c|c|}
\hline type & real subalgebras & \\
\hline$A_{1}$ & $\mathfrak{s l}(2, \mathbb{R}) \oplus \mathfrak{s o}(1,1)$ & $\mathrm{R}$ \\
\hline$A_{1}$ & $\mathfrak{s u}(2)$ & $\mathrm{S}$ \\
& $\mathfrak{s l}(2, \mathbb{R})$ & \\
\hline
\end{tabular}

Type $B_{2}$

Table 6: Maximal subalgebras of $\mathfrak{s o}(2,3)$.

\begin{tabular}{|l|r|l|}
\hline type & real subalgebras & \\
\hline$A_{1}+A_{1}$ & $\mathfrak{s l}(2, \mathbb{R}) \oplus \mathfrak{s l}(2, \mathbb{R})$ & $\mathrm{R}$ \\
& $\mathfrak{s l}(2, \mathbb{C})$ & \\
\hline
\end{tabular}




\begin{tabular}{|l|r|r|}
\hline \multicolumn{3}{|c|}{ subalgebras of $\mathfrak{s o}(2,3)$} \\
\hline & $\mathfrak{s l}(2, \mathbb{R}) \oplus \mathfrak{s o}(1,1)$ & \\
$A_{1}+T_{1}$ & $\mathfrak{s l}(2, \mathbb{R}) \oplus \mathfrak{u}(1)$ & $\mathrm{R}$ \\
& $\mathfrak{s u}(2) \oplus \mathfrak{u}(1)$ & \\
\hline$A_{1}$ & $\mathfrak{s l}(2, \mathbb{R})$ & $\mathrm{S}$ \\
\hline
\end{tabular}

Table 7: Maximal subalgebras of $\mathfrak{s o}(4,1)$.

\begin{tabular}{|l|r|l|}
\hline type & real subalgebras & \\
\hline$A_{1}+A_{1}$ & $\mathfrak{s l}(2, \mathbb{C})$ & $\mathrm{R}$ \\
& $\mathfrak{s u}(2) \oplus \mathfrak{s u}(2)$ & \\
\hline$A_{1}+T_{1}$ & $\mathfrak{s l}(2, \mathbb{R}) \oplus \mathfrak{u}(1)$ & $\mathrm{R}$ \\
& $\mathfrak{s u}(2) \oplus \mathfrak{s o}(1,1)$ & \\
\hline
\end{tabular}

Type $G_{2}$

Table 8: Maximal subalgebras of $G_{2(2)}$.

\begin{tabular}{|c|c|c|}
\hline type & real subalgebras & \\
\hline \multirow{2}{*}{$A_{2}$} & $\mathfrak{s l}(3, \mathbb{R})$ & $\mathrm{P}$ \\
\hline & $\mathfrak{s u}(1,2)$ & К \\
\hline \multirow{2}{*}{$A_{1}+A_{1}$} & $\mathfrak{s l}(2, \mathbb{R}) \oplus \mathfrak{s l}(2, \mathbb{R})$ & \\
\hline & $\mathfrak{s u}(2) \oplus \mathfrak{s u}(2)$ & $\mathrm{R}$ \\
\hline$A_{1}$ & $\mathfrak{s l}(2, \mathbb{R})$ & $\mathrm{S}$ \\
\hline
\end{tabular}

Type $A_{3}$

Table 9: Maximal subalgebras of $\mathfrak{s u}(1,3)$.

\begin{tabular}{|l|c|c|}
\hline type & real subalgebras & \\
\hline$A_{2}$ & $\mathfrak{s u}(1,2) \oplus \mathfrak{u}(1)$ & \\
& $\mathfrak{s u}(3) \oplus \mathfrak{u}(1)$ & $\mathrm{R}$ \\
\hline$A_{1}+A_{1}+T_{1}$ & $\mathfrak{s u}(2) \oplus \mathfrak{s l}(2, \mathbb{R}) \oplus \mathfrak{u}(1)$ & $\mathrm{R}$ \\
\hline
\end{tabular}

Table 10: Maximal subalgebras of $\mathfrak{s u}(2,2)$.

\begin{tabular}{|l|c|l|}
\hline type & real subalgebras & \\
\hline$A_{2}$ & $\mathfrak{s u}(1,2) \oplus \mathfrak{u}(1)(2 \times)$ & $\mathrm{R}$ \\
\hline$A_{1}+A_{1}+T_{1}$ & $\mathfrak{s l}(2, \mathbb{C}) \oplus \mathfrak{s o}(1,1)$ & \\
& $\mathfrak{s u}(2) \oplus \mathfrak{s}) \oplus \mathfrak{s u}(2) \oplus \mathfrak{u}(1)$ & \\
\hline$B_{2}$ & $\mathfrak{s o}(2,3)$ & $\mathrm{S}$ \\
& $\mathfrak{s o}(4,1)$ & \\
\hline$A_{1}+A_{1}$ & $\mathfrak{s u}(2) \oplus \mathfrak{s l}(2, \mathbb{R})$ & $\mathrm{S}$ \\
& $\mathfrak{s l}(2, \mathbb{R}) \oplus \mathfrak{s l}(2, \mathbb{R})$ & \\
\hline
\end{tabular}


Table 11: Maximal subalgebras of $\mathfrak{s l}(2, \mathbb{H})$.

\begin{tabular}{|l|c|l|}
\hline type & real subalgebras & \\
\hline$B_{2}$ & $\mathfrak{s o}(5)$ & $\mathrm{S}$ \\
& $\mathfrak{s o}(4,1)$ & \\
\hline$A_{1}+A_{1}+T_{1}$ & $\mathfrak{s l}(2, \mathbb{C}) \oplus \mathfrak{u}(1)$ & $\mathrm{R}$ \\
& $\mathfrak{s u}(2) \oplus \mathfrak{s u}(2) \oplus \mathfrak{s o}(1,1)$ & \\
\hline$A_{1}+A_{1}$ & $\mathfrak{s u}(2) \oplus \mathfrak{s l}(2, \mathbb{R})$ & $\mathrm{S}$ \\
\hline
\end{tabular}

Table 12: Maximal subalgebras of $\mathfrak{s l}(4, \mathbb{R})$.

\begin{tabular}{|l|r|c|}
\hline type & real subalgebras & \\
\hline$A_{2}$ & $\mathfrak{s l}(3, \mathbb{R}) \oplus \mathfrak{s o}(1,1)$ & $\mathrm{R}$ \\
\hline$A_{1}+A_{1}+T_{1}$ & $\mathfrak{s l}(2, \mathbb{R}) \oplus \mathfrak{s l}(2, \mathbb{R}) \oplus \mathfrak{s o}(1,1)$ & $\mathrm{R}$ \\
& $\mathfrak{s l}(2, \mathbb{C}) \oplus \mathfrak{u}(1)(2 \times)$ & \\
\hline$B_{2}$ & $\mathfrak{s o}(2,3)$ & $\mathrm{S}$ \\
\hline \multirow{2}{*}{$A_{1}+A_{1}$} & $\mathfrak{s u}(2) \oplus \mathfrak{s u}(2)$ & $\mathrm{S}$ \\
& $\mathfrak{s l}(2, \mathbb{R}) \oplus \mathfrak{s l}(2, \mathbb{R})$ & \\
\hline
\end{tabular}

Type $B_{3}$

Table 13: Maximal subalgebras of $\mathfrak{s o}(2,5)$.

\begin{tabular}{|l|r|l|}
\hline type & real subalgebras & \\
\hline \multirow{3}{*}{$A_{1}+A_{1}+A_{1}$} & $\mathfrak{s u}(2) \oplus \mathfrak{s l}(2, \mathbb{R}) \oplus \mathfrak{s l}(2, \mathbb{R})$ & \\
& $\mathfrak{s l}(2, \mathbb{C}) \oplus \mathfrak{s l}(2, \mathbb{R})$ & $\mathrm{R}$ \\
& $\mathfrak{s u}(2) \oplus \mathfrak{s u}(2) \oplus \mathfrak{s l}(2, \mathbb{R})$ & \\
\hline \multirow{2}{*}{$A_{3}$} & $\mathfrak{s u}(2,2)$ & $\mathrm{R}$ \\
\hline \multirow{3}{*}{$B_{2}+T_{1}$} & $\mathfrak{s l}(2, \mathbb{H})$ & \\
& $\mathfrak{s o}(4,1) \oplus \mathfrak{s o}(1,1)$ & \\
& $\mathfrak{s o}(2,3) \oplus \mathfrak{u}(1)$ & $\mathrm{R}$ \\
& $\mathfrak{s o}(5) \oplus \mathfrak{u}(1)$ & \\
\hline
\end{tabular}

Table 14: Maximal subalgebras of $\mathfrak{s o}(4,3)$.

\begin{tabular}{|l|r|l|}
\hline type & real subalgebras & \\
\hline & $\mathfrak{s l}(2, \mathbb{R}) \oplus \mathfrak{s l}(2, \mathbb{R}) \oplus \mathfrak{s l}(2, \mathbb{R})$ & \\
$A_{1}+A_{1}+A_{1}$ & $\mathfrak{s l}(2, \mathbb{C}) \oplus \mathfrak{s l}(2, \mathbb{R})$ & \\
& $\mathfrak{s l}(2, \mathbb{C}) \oplus \mathfrak{s u}(2)$ & $\mathrm{R}$ \\
& $\mathfrak{s u}(2) \oplus \mathfrak{s u}(2) \oplus \mathfrak{s u}(2)$ & \\
\hline \multirow{2}{*}{$A_{3}$} & $\mathfrak{s l}(4, \mathbb{R})$ & $\mathrm{R}$ \\
\hline & $\mathfrak{s u}(2,2)$ & \\
$B_{2}+T_{1}$ & $\mathfrak{s o}(2,3) \oplus \mathfrak{s o}(1,1)$ & $\mathrm{R}$ \\
& $\mathfrak{s o}(2,3) \oplus \mathfrak{u}(1)$ & \\
\hline$G_{2}$ & $\mathfrak{s o}(4,1) \oplus \mathfrak{u}(1)$ & $\mathrm{S}$ \\
\hline
\end{tabular}


Table 15: Maximal subalgebras of $\mathfrak{s o}(6,1)$.

\begin{tabular}{|l|c|l|}
\hline type & real subalgebras & \\
\hline \multirow{2}{*}{$A_{1}+A_{1}+A_{1}$} & $\mathfrak{s l}(2, \mathbb{C}) \oplus \mathfrak{s u}(2)$ & $\mathrm{R}$ \\
& $\mathfrak{s u}(2) \oplus \mathfrak{s u}(2) \oplus \mathfrak{s l}(2, \mathbb{R})$ & \\
\hline \multirow{2}{*}{$A_{3}$} & $\mathfrak{s l}(2, \mathbb{H})$ & $\mathrm{R}$ \\
\hline \multirow{2}{*}{$B_{2}+T_{1}$} & $\mathfrak{s u}(4)$ & \\
\hline & $\mathfrak{s o}(5) \oplus \mathfrak{s o}(1,1)$ & $\mathrm{R}$ \\
\hline
\end{tabular}

Type $C_{3}$

Table 16: Maximal subalgebras of $\mathfrak{s p}(1,2)$.

\begin{tabular}{|l|c|c|}
\hline type & real subalgebras & \\
\hline$A_{2}$ & $\mathfrak{s u}(1,2) \oplus \mathfrak{u}(1)$ & $\mathrm{R}$ \\
\hline$A_{1}+B_{2}$ & $\begin{array}{r}\mathfrak{s u}(2) \oplus \mathfrak{s o}(4,1) \\
\mathfrak{s u}(2) \oplus \mathfrak{s o}(5)\end{array}$ & $\mathrm{R}$ \\
\hline$A_{1}+A_{1}$ & $\mathfrak{s u}(2) \oplus \mathfrak{s l}(2, \mathbb{R})$ & $\mathrm{S}$ \\
\hline
\end{tabular}

Table 17: Maximal subalgebras of $\mathfrak{s p}(3, \mathbb{R})$.

\begin{tabular}{|l|r|l|}
\hline type & real subalgebras & \\
\hline & $\mathfrak{s l}(3, \mathbb{R}) \oplus \mathfrak{s o}(1,1)$ & \\
$A_{2}$ & $\mathfrak{s u}(1,2) \oplus \mathfrak{u}(1)$ & $\mathrm{R}$ \\
& $\mathfrak{s u}(3) \oplus \mathfrak{u}(1)$ & \\
\hline$A_{1}+B_{2}$ & $\mathfrak{s l}(2, \mathbb{R}) \oplus \mathfrak{s o}(2,3)$ & $\mathrm{R}$ \\
\hline$A_{1}$ & $\mathfrak{s l}(2, \mathbb{R})$ & $\mathrm{S}$ \\
\hline$A_{1}+A_{1}$ & $\mathfrak{s l}(2, \mathbb{R}) \oplus \mathfrak{s l}(2, \mathbb{R})$ & $\mathrm{S}$ \\
\hline
\end{tabular}

Type $A_{4}$

Table 18: Maximal subalgebras of $\mathfrak{s u}(1,4)$.

\begin{tabular}{|l|c|l|}
\hline type & real subalgebras & \\
\hline$A_{1}+A_{2}+T_{1}$ & $\begin{array}{c}\mathfrak{s u}(2) \oplus \mathfrak{s u}(1,2) \oplus \mathfrak{u}(1) \\
\mathfrak{s l}(2, \mathbb{R}) \oplus \mathfrak{s u}(3) \oplus \mathfrak{u}(1)\end{array}$ & $\mathrm{R}$ \\
\hline \multirow{2}{*}{$A_{3}+T_{1}$} & $\mathfrak{s u}(1,3) \oplus \mathfrak{u}(1)$ & $\mathrm{R}$ \\
\hline$B_{2}$ & $\mathfrak{s u}(4) \oplus \mathfrak{u}(1)$ & \\
\hline
\end{tabular}

Table 19: Maximal subalgebras of $\mathfrak{s u}(2,3)$.

\begin{tabular}{|l|c|l|}
\hline type & real subalgebras & \\
\hline & $\mathfrak{s l}(2, \mathbb{R}) \oplus \mathfrak{s u}(1,2) \oplus \mathfrak{u}(1)$ & \\
$A_{1}+A_{2}+T_{1}$ & $\mathfrak{s u}(2) \oplus \mathfrak{s u}(1,2) \oplus \mathfrak{u}(1)$ & $\mathrm{R}$ \\
& $\mathfrak{s u}(2) \oplus \mathfrak{s u}(3) \oplus \mathfrak{u}(1)$ & \\
\hline \multirow{2}{*}{$A_{3}+T_{1}$} & $\mathfrak{s u}(2,2) \oplus \mathfrak{u}(1)$ & $\mathrm{R}$ \\
& $\mathfrak{s u}(1,3) \oplus \mathfrak{u}(1)$ & \\
\hline$B_{2}$ & $\mathfrak{s o}(2,3)$ & $\mathrm{S}$ \\
\hline
\end{tabular}


Table 20: Maximal subalgebras of $\mathfrak{s l}(5, \mathbb{R})$.

\begin{tabular}{|l|c|c|}
\hline type & real subalgebras & \\
\hline$A_{1}+A_{2}+T_{1}$ & $\mathfrak{s l}(2, \mathbb{R}) \oplus \mathfrak{s l}(3, \mathbb{R}) \oplus \mathfrak{s o}(1,1)$ & $\mathrm{R}$ \\
\hline$A_{3}+T_{1}$ & $\mathfrak{s l}(4, \mathbb{R}) \oplus \mathfrak{s o}(1,1)$ & $\mathrm{R}$ \\
\hline & $\mathfrak{s o}(5)$ & \\
$B_{2}$ & $\mathfrak{s o}(2,3)$ & $\mathrm{S}$ \\
& $\mathfrak{s o}(4,1)$ & \\
\hline
\end{tabular}

Type $B_{4}$

Table 21: Maximal subalgebras of $\mathfrak{s o}(2,7)$.

\begin{tabular}{|l|r|l|}
\hline type & real subalgebras & \\
\hline & $\mathfrak{s l}(2, \mathbb{R}) \oplus \mathfrak{s l}(2, \mathbb{R}) \oplus \mathfrak{s o}(5)$ & \\
$A_{1}+A_{1}+B_{2}$ & $\mathfrak{s u}(2) \oplus \mathfrak{s u}(2) \oplus \mathfrak{s o}(2,3)$ & $\mathrm{R}$ \\
& $\mathfrak{s l}(2, \mathbb{C}) \oplus \mathfrak{s o}(4,1)$ & \\
\hline \multirow{3}{*}{$A_{1}+A_{3}$} & $\mathfrak{s u}(2) \oplus \mathfrak{s u}(2,2)$ & \\
& $\mathfrak{s l}(2, \mathbb{R}) \oplus \mathfrak{s l}(2, \mathbb{H})$ & $\mathrm{R}$ \\
& $\mathfrak{s l}(2, \mathbb{R}) \oplus \mathfrak{s u}(4)$ & \\
\hline \multirow{3}{*}{$B_{3}+T_{1}$} & $\mathfrak{s o}(6,1) \oplus \mathfrak{s o}(1,1)$ & \\
& $\mathfrak{s o}(2,5) \oplus \mathfrak{u}(1)$ & $\mathrm{R}$ \\
& $\mathfrak{s o}(7) \oplus \mathfrak{u}(1)$ & \\
\hline \multirow{2}{*}{$D_{4}$} & $\mathfrak{s o}(8)$ & $\mathrm{R}$ \\
& $\mathfrak{s o}(1,7)$ & \\
\hline
\end{tabular}

Table 22: Maximal subalgebras of $\mathfrak{s o}(4,5)$.

\begin{tabular}{|l|r|l|}
\hline type & real subalgebras & \\
\hline & $\mathfrak{s l}(2, \mathbb{R}) \oplus \mathfrak{s l}(2, \mathbb{R}) \oplus \mathfrak{s o}(2,3)$ & \\
& $\mathfrak{s l}(2, \mathbb{C}) \oplus \mathfrak{s o}(2,3)$ & \\
$A_{1}+A_{1}+B_{2}$ & $\mathfrak{s l}(2, \mathbb{C}) \oplus \mathfrak{s o}(4,1)$ & $\mathrm{R}$ \\
& $\mathfrak{s u}(2) \oplus \mathfrak{s u}(2) \oplus \mathfrak{s o}(4,1)$ & \\
& $\mathfrak{s u}(2) \oplus \mathfrak{s u}(2) \oplus \mathfrak{s o}(5)$ & \\
\hline & $\mathfrak{s l}(2, \mathbb{R}) \oplus \mathfrak{s l}(4, \mathbb{R})$ & \\
& $\mathfrak{s l}(2, \mathbb{R}) \oplus \mathfrak{s u}(2,2)$ & \\
$A_{1}+A_{3}$ & $\mathfrak{s u}(2) \oplus \mathfrak{s u}(2,2)$ & $\mathrm{R}$ \\
& $\mathfrak{s u}(2) \oplus \mathfrak{s l}(2, \mathbb{H})$ & \\
\hline \multirow{2}{*}{$D_{4}$} & $\mathfrak{s o}(4,4)$ & $\mathrm{R}$ \\
\hline & $\mathfrak{s o}(3,5)$ & \\
$B_{3}+T_{1}$ & $\mathfrak{s o}(4,3) \oplus \mathfrak{s o}(1,1)$ & $\mathrm{R}$ \\
& $\mathfrak{s o}(4,3) \oplus \mathfrak{u}(1)$ & $\mathrm{S}$ \\
\hline$A_{1}$ & $\mathfrak{s o}(2,5) \oplus \mathfrak{u}(1)$ & $\mathrm{S}$ \\
\hline$A_{1}+A_{1}$ & $\mathfrak{s l}(2, \mathbb{R})$ & \\
\hline
\end{tabular}


Table 23: Maximal subalgebras of $\mathfrak{s o}(6,3)$.

\begin{tabular}{|l|r|l|}
\hline type & real subalgebras & \\
\hline & $\mathfrak{s l}(2, \mathbb{R}) \oplus \mathfrak{s l}(2, \mathbb{R}) \oplus \mathfrak{s o}(4,1)$ & \\
$A_{1}+A_{1}+B_{2}$ & $\mathfrak{s l}(2, \mathbb{C}) \oplus \mathfrak{s o}(2,3)$ & \\
& $\mathfrak{s u}(2) \oplus \mathfrak{s u}(2) \oplus \mathfrak{s o}(2,3)$ & $\mathrm{R}$ \\
& $\mathfrak{s l}(2, \mathbb{C}) \oplus \mathfrak{s o}(5)$ & \\
\hline & $\mathfrak{s u}(2) \oplus \mathfrak{s l}(4, \mathbb{R})$ & \\
$A_{1}+A_{3}$ & $\mathfrak{s l}(2, \mathbb{R}) \oplus \mathfrak{s u}(2,2)$ & $\mathrm{R}$ \\
& $\mathfrak{s l}(2, \mathbb{R}) \oplus \mathfrak{s l}(2, \mathbb{H})$ & \\
& $\mathfrak{s u}(2) \oplus \mathfrak{s u}(4)$ & \\
\hline$D_{4}$ & $\mathfrak{s o}(3,5)$ & $\mathrm{R}$ \\
\hline & $\mathfrak{s o}(8)$ & $\mathrm{R}$ \\
$B_{3}+T_{1}$ & $\mathfrak{s o}(2,5) \oplus \mathfrak{s o}(1,1)$ & \\
\hline$A_{1}+A_{1}$ & $\mathfrak{s o}(4,3) \oplus \mathfrak{u}(1)$ & $\mathrm{S}$ \\
\hline
\end{tabular}

Table 24: Maximal subalgebras of $\mathfrak{s o}(8,1)$.

\begin{tabular}{|l|c|l|}
\hline type & real subalgebras & \\
\hline$A_{1}+A_{1}+B_{2}$ & $\begin{array}{r}\mathfrak{s l}(2, \mathbb{C}) \oplus \mathfrak{s o}(5) \\
\mathfrak{s u}(2) \oplus \mathfrak{s u}(2) \oplus \mathfrak{s o}(4,1)\end{array}$ & $\mathrm{R}$ \\
\hline$A_{1}+A_{3}$ & $\begin{array}{c}\mathfrak{s u}(2) \oplus \mathfrak{s l}(2, \mathbb{H}) \\
\mathfrak{s l}(2, \mathbb{R}) \oplus \mathfrak{s u}(4)\end{array}$ & $\mathrm{R}$ \\
\hline$D_{4}$ & $\begin{array}{c}\mathfrak{s o}(1,7) \\
\mathfrak{s o}(8)\end{array}$ & $\mathrm{R}$ \\
\hline$B_{3}+T_{1}$ & $\begin{array}{c}\mathfrak{s o}(7) \oplus \mathfrak{s o}(1,1) \\
\mathfrak{s o}(6,1) \oplus \mathfrak{u}(1)\end{array}$ & $\mathrm{R}$ \\
\hline
\end{tabular}

Type $C_{4}$

Table 25: Maximal subalgebras of $\mathfrak{s p}(1,3)$.

\begin{tabular}{|c|c|c|}
\hline type & real subalgebras & \\
\hline$A_{3}+T_{1}$ & $\mathfrak{s \mathfrak { u } ( 1 , 3 ) \oplus \mathfrak { u } ( 1 )}$ & $\mathrm{R}$ \\
\hline$B_{2}+B_{2}$ & $\mathfrak{s o}(5) \oplus \mathfrak{s o}(4,1)$ & $\mathrm{R}$ \\
\hline$A_{1}+C_{3}$ & $\begin{array}{r}\mathfrak{s u}(2) \oplus \mathfrak{s p}(1,2) \\
\mathfrak{s u}(2) \oplus \mathfrak{s p}(3)\end{array}$ & $\mathrm{R}$ \\
\hline
\end{tabular}

Table 26: Maximal subalgebras of $\mathfrak{s p}(2,2)$.

\begin{tabular}{|l|r|l|}
\hline type & real subalgebras & \\
\hline$A_{3}+T_{1}$ & $\mathfrak{s l}(2, \mathbb{H}) \oplus \mathfrak{s o}(1,1)$ & $\mathrm{R}$ \\
& $\mathfrak{s u}(2,2) \oplus \mathfrak{u}(1)$ & \\
\hline \multirow{3}{*}{$B_{2}+B_{2}$} & $\mathfrak{s o}(4,1) \oplus \mathfrak{s o}(4,1)$ & \\
& $\mathfrak{s o}(5, \mathbb{C})$ & $\mathrm{R}$ \\
& $\mathfrak{s o}(5) \oplus \mathfrak{s o}(5)$ & \\
\hline
\end{tabular}




\begin{tabular}{|l|c|c|}
\hline \multicolumn{4}{|l|}{ subalgebras of $\mathfrak{s p}(2,2)$} \\
\hline$A_{1}+C_{3}$ & $\mathfrak{s u}(2) \oplus \mathfrak{s p}(1,2)(2 \times)$ & $\mathrm{R}$ \\
\hline$A_{1}+A_{1}+A_{1}$ & $\mathfrak{s u}(2) \oplus \mathfrak{s l}(2, \mathbb{R}) \oplus \mathfrak{s l}(2, \mathbb{R})$ & $\mathrm{S}$ \\
\hline
\end{tabular}

Table 27: Maximal subalgebras of $\mathfrak{s p}(4, \mathbb{R})$.

\begin{tabular}{|l|c|c|}
\hline type & real subalgebras & \\
\hline & $\mathfrak{s l}(4, \mathbb{R}) \oplus \mathfrak{s o}(1,1)$ & \\
$A_{3}+T_{1}$ & $\mathfrak{s u}(2,2) \oplus \mathfrak{u}(1)$ & \multirow{2}{*}{$\begin{array}{l} \\
\end{array}$} \\
& $\mathfrak{s u}(1,3) \oplus \mathfrak{u}(1) \oplus \mathfrak{u}(1)$ & \\
\hline$B_{2}+B_{2}$ & $\mathfrak{s o}(2,3) \oplus \mathfrak{s o}(2,3)$ & $\mathrm{R}$ \\
& $\mathfrak{s o}(5, \mathbb{C})$ & \\
\hline$A_{1}+C_{3}$ & $\mathfrak{s l}(2, \mathbb{R}) \oplus \mathfrak{s p}(3, \mathbb{R})$ & $\mathrm{R}$ \\
\hline$A_{1}$ & $\mathfrak{s l}(2, \mathbb{R})$ & $\mathrm{S}$ \\
\hline$A_{1}+A_{1}+A_{1}$ & $\mathfrak{s l}(2, \mathbb{R}) \oplus \mathfrak{s l}(2, \mathbb{R}) \oplus \mathfrak{s l}(2, \mathbb{R})$ & $\mathrm{S}$ \\
\hline
\end{tabular}

Type $D_{4}$

Table 28: Maximal subalgebras of $\mathfrak{s o}^{*}(8)$.

\begin{tabular}{|l|c|l|}
\hline type & real subalgebras & \\
\hline$A_{1}+A_{1}+A_{1}+A_{1}$ & $\mathfrak{s u}(2) \oplus \mathfrak{s u}(2) \oplus \mathfrak{s l}(2, \mathbb{R}) \oplus \mathfrak{s l}(2, \mathbb{R})$ & $\mathrm{R}$ \\
& $\mathfrak{s l}(2, \mathbb{C}) \oplus \mathfrak{s l}(2, \mathbb{C})$ & \\
$A_{3}+T_{1}$ & $\mathfrak{s u}(2,2) \oplus \mathfrak{u}(1)$ & \\
& $\mathfrak{s l}(2, \mathbb{H}) \oplus \mathfrak{s o}(1,1)$ & $\mathrm{R}$ \\
\hline \multirow{2}{*}{$A_{2}$} & $\mathfrak{s u}(4) \oplus \mathfrak{u}(1)$ & \\
& $\mathfrak{s u}(2) \oplus \mathfrak{s o}(2,3)$ & $\mathrm{S}$ \\
& $\mathfrak{s l}(2, \mathbb{R}) \oplus \mathfrak{s o}(5)$ & \\
\hline$B_{3}$ & $\mathfrak{s l}(2, \mathbb{R}) \oplus \mathfrak{s o}(4,1)$ & $\mathrm{S}$ \\
\hline
\end{tabular}

Table 29: Maximal subalgebras of $\mathfrak{s o}(4,4)$.

\begin{tabular}{|l|r|l|}
\hline type & real subalgebras & \\
\hline & $\mathfrak{s l}(2, \mathbb{R}) \oplus \mathfrak{s l}(2, \mathbb{R}) \oplus \mathfrak{s l}(2, \mathbb{R}) \oplus \mathfrak{s l}(2, \mathbb{R})$ & \\
$A_{1}+A_{1}+A_{1}+A_{1}$ & $\mathfrak{s l}(2, \mathbb{C}) \oplus \mathfrak{s l}(2, \mathbb{C})(3 \times)$ & $\mathrm{R}$ \\
& $\mathfrak{s u}(2) \oplus \mathfrak{s u}(2) \oplus \mathfrak{s u}(2) \oplus \mathfrak{s u}(2)$ & \\
\hline$A_{3}+T_{1}$ & $\mathfrak{s l}(4, \mathbb{R}) \oplus \mathfrak{s o}(1,1)$ & $\mathrm{R}$ \\
\hline$A_{2}$ & $\mathfrak{s u}(2,2) \oplus \mathfrak{u}(1)(6 \times)$ & $\mathrm{S}$ \\
\hline$A_{1}+B_{2}$ & $\mathfrak{s u}(1,2)$ & $\mathrm{S}$ \\
\hline$B_{3}$ & $\mathfrak{s u}(2) \oplus \mathfrak{s o}(4,1)$ & $\mathrm{S}$ \\
\hline
\end{tabular}


Table 30: Maximal subalgebras of $\mathfrak{s o}(3,5)$.

\begin{tabular}{|l|c|c|}
\hline type & real subalgebras & \\
\hline$A_{1}+A_{1}+A_{1}+A_{1}$ & $\mathfrak{s l}(2, \mathbb{C}) \oplus \mathfrak{s l}(2, \mathbb{R}) \oplus \mathfrak{s l}(2, \mathbb{R})$ & $\mathrm{R}$ \\
& $\mathfrak{s l}(2, \mathbb{C}) \oplus \mathfrak{s u}(2) \oplus \mathfrak{s u}(2)$ & \\
\hline \multirow{3}{*}{$A_{3}+T_{1}$} & $\mathfrak{s l}(4, \mathbb{R}) \oplus \mathfrak{u}(1)$ & \\
& $\mathfrak{s u}(2,2) \oplus \mathfrak{s o}(1,1)$ & $\mathrm{R}$ \\
\hline$A_{2}$ & $\mathfrak{s l}(2, \mathbb{H}) \oplus \mathfrak{u}(1)$ & \\
\hline & $\mathfrak{s l}(3, \mathbb{R})$ & $\mathrm{S}$ \\
\hline \multirow{2}{*}{$A_{1}+B_{2}$} & $\mathfrak{s u}(2) \oplus \mathfrak{s o}(5)$ & \\
& $\mathfrak{s u}(2) \oplus \mathfrak{s o}(2,3)$ & $\mathrm{S}$ \\
& $\mathfrak{s l}(2, \mathbb{R}) \oplus \mathfrak{s o}(2,3)$ & \\
\hline \multirow{2}{*}{$B_{3}$} & $\mathfrak{s l}(2, \mathbb{R}) \oplus \mathfrak{s o}(4,1)$ & \\
& $\mathfrak{s o}(2,5)$ & $\mathrm{S}$ \\
\hline
\end{tabular}

Table 31: Maximal subalgebras of $\mathfrak{s o}(1,7)$.

\begin{tabular}{|l|c|c|}
\hline type & real subalgebras & \\
\hline$A_{1}+A_{1}+A_{1}+A_{1}$ & $\mathfrak{s l}(2, \mathbb{C}) \oplus \mathfrak{s u}(2) \oplus \mathfrak{s u}(2)$ & $\mathrm{R}$ \\
\hline$A_{3}+T_{1}$ & $\mathfrak{s l}(2, \mathbb{H}) \oplus \mathfrak{u}(1)$ & $\mathrm{R}$ \\
& $\mathfrak{s u}(4) \oplus \mathfrak{s o}(1,1)$ & \\
\hline$A_{1}+B_{2}$ & $\begin{array}{c}\mathfrak{s u}(2) \oplus \mathfrak{s o}(4,1) \\
\mathfrak{s l}(2, \mathbb{R}) \oplus \mathfrak{s o}(5)\end{array}$ & $\mathrm{S}$ \\
\hline$B_{3}$ & $\begin{array}{c}\mathfrak{s o}(7) \\
\mathfrak{s o}(6,1)\end{array}$ & $\mathrm{S}$ \\
\hline
\end{tabular}

Type $F_{4}$

Table 32: Maximal subalgebras of $F_{4(4)}$.

\begin{tabular}{|l|r|l|}
\hline type & real subalgebras & \\
\hline & $\mathfrak{s l}(3, \mathbb{R}) \oplus \mathfrak{s l}(3, \mathbb{R})$ & \\
$A_{2}+A_{2}$ & $\mathfrak{s u}(1,2) \oplus \mathfrak{s u}(1,2)$ & $\mathrm{R}$ \\
& $\mathfrak{s u}(3) \oplus \mathfrak{s u}(1,2)$ & \\
\hline & $\mathfrak{s l}(2, \mathbb{R}) \oplus \mathfrak{s p}(3, \mathbb{R})$ & \\
$A_{1}+C_{3}$ & $\mathfrak{s u}(2) \oplus \mathfrak{s p}(1,2)$ & $\mathrm{R}$ \\
& $\mathfrak{s u}(2) \oplus \mathfrak{s p}(3)$ & \\
\hline$B_{4}$ & $\mathfrak{s o}(4,5)$ & $\mathrm{R}$ \\
\hline$A_{1}$ & $\mathfrak{s l}(2, \mathbb{R})$ & $\mathrm{S}$ \\
\hline \multirow{2}{*}{$A_{1}+G_{2}$} & $\mathfrak{s u}(2) \oplus G_{2(2)}$ & $\mathrm{S}$ \\
& $\mathfrak{s l}(2, \mathbb{R}) \oplus G_{2(2)}$ & \\
\hline
\end{tabular}

Table 33: Maximal subalgebras of $F_{4(-20)}$.

\begin{tabular}{|l|c|c|}
\hline type & real subalgebras & \\
\hline$A_{2}+A_{2}$ & $\mathfrak{s u}(3) \oplus \mathfrak{s u}(1,2)$ & $\mathrm{R}$ \\
\hline$A_{1}+C_{3}$ & $\mathfrak{s u}(2) \oplus \mathfrak{s p}(1,2)$ & $\mathrm{R}$ \\
\hline
\end{tabular}




\begin{tabular}{|l|c|c|}
\hline \multicolumn{3}{|c|}{ subalgebras of $F_{4(-20)}$} \\
\hline$B_{4}$ & $\mathfrak{s o}(8,1)$ & $\mathrm{R}$ \\
& $\mathfrak{s o}(9)$ & \\
\hline$A_{1}+G_{2}$ & $\mathfrak{s l}(2, \mathbb{R}) \oplus G_{2}^{c}$ & $\mathrm{~S}$ \\
\hline
\end{tabular}

Type $A_{5}$

Table 34: Maximal subalgebras of $\mathfrak{s u}(1,5)$.

\begin{tabular}{|l|c|c|}
\hline type & real subalgebras & \\
\hline$A_{2}+A_{2}+T_{1}$ & $\mathfrak{s u}(3) \oplus \mathfrak{s u}(1,2) \oplus \mathfrak{u}(1)$ & $\mathrm{R}$ \\
\hline$A_{1}+A_{3}+T_{1}$ & $\begin{array}{c}\mathfrak{s u}(2) \oplus \mathfrak{s u}(1,3) \oplus \mathfrak{u}(1) \\
\mathfrak{s l}(2, \mathbb{R}) \oplus \mathfrak{s u}(4) \oplus \mathfrak{u}(1)\end{array}$ & $\mathrm{R}$ \\
\hline$A_{4}+T_{1}$ & $\begin{array}{c}\mathfrak{s u}(1,4) \oplus \mathfrak{u}(1) \\
\mathfrak{s u}(5) \oplus \mathfrak{u}(1)\end{array}$ & $\mathrm{R}$ \\
\hline$A_{3}$ & $\mathfrak{s l}(2, \mathbb{H})$ & $\mathrm{S}$ \\
\hline
\end{tabular}

Table 35: Maximal subalgebras of $\mathfrak{s u}(2,4)$.

\begin{tabular}{|c|c|c|}
\hline type & real subalgebras & \\
\hline \multirow{2}{*}{$A_{2}+A_{2}+T_{1}$} & $\mathfrak{s u}(1,2) \oplus \mathfrak{s u}(1,2) \oplus \mathfrak{u}(1)$ & \multirow{2}{*}{$\mathrm{R}$} \\
\hline & $\mathfrak{s u}(3) \oplus \mathfrak{s u}(1,2) \oplus \mathfrak{u}(1)$ & \\
\hline \multirow{3}{*}{$A_{1}+A_{3}+T_{1}$} & $\overline{\mathfrak{s u}}(2) \oplus \mathfrak{s u}(2,2) \oplus \mathfrak{u}(1)$ & \multirow{3}{*}{$\mathrm{R}$} \\
\hline & $\mathfrak{s l}(2, \mathbb{R}) \oplus \mathfrak{s u}(1,3) \oplus \mathfrak{u}(1)$ & \\
\hline & $\mathfrak{s u}(2) \oplus \mathfrak{s u}(4) \oplus \mathfrak{u}(1)$ & \\
\hline \multirow{2}{*}{$A_{4}+T_{1}$} & $\mathfrak{s u}(2,3) \oplus \mathfrak{u}(1)$ & \multirow{2}{*}{$\mathrm{R}$} \\
\hline & $\mathfrak{s u}(1,4) \oplus \mathfrak{u}(1)$ & \\
\hline$A_{2}$ & $\mathfrak{s u}(1,2)$ & $\mathrm{S}$ \\
\hline$A_{1}+A_{2}$ & $\mathfrak{s u}(2) \oplus \mathfrak{s u}(1,2)$ & $\mathrm{S}$ \\
\hline$A_{3}$ & $\mathfrak{s u}(2,2)$ & $\bar{S}$ \\
\hline$C_{3}$ & $\frac{\mathfrak{s p}(1,2)}{}$ & $\mathrm{S}$ \\
\hline
\end{tabular}

Table 36: Maximal subalgebras of $\mathfrak{s u}(3,3)$.

\begin{tabular}{|l|c|l|}
\hline type & real subalgebras & \\
\hline$A_{2}+A_{2}+T_{1}$ & $\begin{array}{r}\mathfrak{s l}(3, \mathbb{C}) \oplus \mathfrak{s}(1,1) \\
\mathfrak{s u}(1,2) \oplus \mathfrak{s u}(1,2) \oplus \mathfrak{u}(1) \\
\mathfrak{s u}(3) \oplus \mathfrak{s u}(3) \oplus \mathfrak{u}(1)\end{array}$ & $\mathrm{R}$ \\
\hline$A_{1}+A_{3}+T_{1}$ & $\begin{array}{r}\mathfrak{s l}(2, \mathbb{R}) \oplus \mathfrak{s u}(2,2) \oplus \mathfrak{u}(1) \\
\mathfrak{s u}(2) \oplus \mathfrak{s u}(1,3) \oplus \mathfrak{u}(1)(2 \times)\end{array}$ & $\mathrm{R}$ \\
\hline$A_{4}+T_{1}$ & $\mathfrak{s u}(2,3) \oplus \mathfrak{u}(1)(2 \times)$ & $\mathrm{R}$ \\
\hline$A_{1}+A_{2}$ & $\begin{array}{r}\mathfrak{s l}(2, \mathbb{R}) \oplus \mathfrak{s u}(3) \\
\text { sl }(2, \mathbb{R}) \oplus \mathfrak{s u}(1,2)\end{array}$ & $\mathrm{S}$ \\
\hline$A_{3}$ & $\mathfrak{s u}(1,3)$ & $\mathrm{S}$ \\
\hline$C_{3}$ & $\mathfrak{s l}(4, \mathbb{R})$ & $\mathrm{S}$ \\
\hline
\end{tabular}


Table 37: Maximal subalgebras of $\mathfrak{s l}(3, \mathbb{H})$.

\begin{tabular}{|l|c|c|}
\hline type & real subalgebras & \\
\hline$A_{2}+A_{2}+T_{1}$ & $\mathfrak{s l}(3, \mathbb{C}) \oplus \mathfrak{u}(1)$ & $\mathrm{S}$ \\
\hline$A_{1}+A_{3}+T_{1}$ & $\mathfrak{s u}(2) \oplus \mathfrak{s l}(2, \mathbb{H}) \oplus \mathfrak{s o}(1,1)$ & $\mathrm{R}$ \\
\hline$A_{1}+A_{2}$ & $\mathfrak{s u}(2) \oplus \mathfrak{s l}(3, \mathbb{R})$ & $\mathrm{S}$ \\
\hline$A_{3}$ & $\mathfrak{s u}(1,3)$ & $\mathrm{S}$ \\
\hline$C_{3}$ & $\mathfrak{s p}(3)$ & $\mathrm{S}$ \\
& $\mathfrak{s p}(1,2)$ & \\
\hline
\end{tabular}

Table 38: Maximal subalgebras of $\mathfrak{s l}(6, \mathbb{R})$.

\begin{tabular}{|l|c|c|}
\hline type & real subalgebras & \\
\hline$A_{2}+A_{2}+T_{1}$ & $\mathfrak{s l}(3, \mathbb{R}) \oplus \mathfrak{s l}(3, \mathbb{R}) \oplus \mathfrak{s o}(1,1)$ & $\mathrm{R}$ \\
& $\mathfrak{s l}(3, \mathbb{C}) \oplus \mathfrak{u}(1)$ & \\
\hline$A_{1}+A_{3}+T_{1}$ & $\mathfrak{s l}(2, \mathbb{R}) \oplus \mathfrak{s l}(4, \mathbb{R}) \oplus \mathfrak{s o}(1,1)$ & $\mathrm{R}$ \\
\hline$A_{4}+T_{1}$ & $\mathfrak{s l}(5, \mathbb{R}) \oplus \mathfrak{s o}(1,1)$ & $\mathrm{R}$ \\
\hline$A_{2}$ & $\mathfrak{s l}(3, \mathbb{R})$ & $\mathrm{S}$ \\
\hline$A_{1}+A_{2}$ & $\mathfrak{s l}(2, \mathbb{R}) \oplus \mathfrak{s l}(3, \mathbb{R})$ & $\mathrm{S}$ \\
\hline & $\mathfrak{s u}(4)$ & \\
& $\mathfrak{s u}(2,2)$ & $\mathrm{S}$ \\
$A_{3}$ & $\mathfrak{s l}(2, \mathbb{H})$ & \\
& $\mathfrak{s l}(4, \mathbb{R})$ & $\mathrm{S}$ \\
\hline$C_{3}$ & $\mathfrak{s p}(3, \mathbb{R})$ & \\
\hline
\end{tabular}

Type $B_{5}$

Table 39: Maximal subalgebras of $\mathfrak{s o}(2,9)$.

\begin{tabular}{|l|r|l|}
\hline type & real subalgebras & \\
\hline \multirow{3}{*}{$A_{3}+B_{2}$} & $\mathfrak{s u}(2,2) \oplus \mathfrak{s o}(5)$ & \\
& $\mathfrak{s l}(2, \mathbb{H}) \oplus \mathfrak{s o}(4,1)$ & $\mathrm{R}$ \\
& $\mathfrak{s u}(4) \oplus \mathfrak{s o}(2,3)$ & \\
\hline \multirow{3}{*}{$A_{1}+A_{1}+B_{3}$} & $\mathfrak{s l}(2, \mathbb{R}) \oplus \mathfrak{s l}(2, \mathbb{R}) \oplus \mathfrak{s o}(7)$ & \\
& $\mathfrak{s l}(2, \mathbb{C}) \oplus \mathfrak{s o}(6,1)$ & $\mathrm{R}$ \\
& $\mathfrak{s u}(2) \oplus \mathfrak{s u}(2) \oplus \mathfrak{s o}(2,5)$ & \\
\hline \multirow{3}{*}{$A_{1}+D_{4}$} & $\mathfrak{s u}(2) \oplus \mathfrak{s o}(8)$ & \\
& $\mathfrak{s l}(2, \mathbb{R}) \oplus \mathfrak{s o}(1,7)$ & $\mathrm{R}$ \\
& $\mathfrak{s l}(2, \mathbb{R}) \oplus \mathfrak{s o}(8)$ & \\
\hline$D_{5}$ & $\mathfrak{s o}(2,8)$ & $\mathrm{R}$ \\
\hline & $\mathfrak{s o}(9,1)$ & \\
$B_{4}+T_{1}$ & $\mathfrak{s o}(8,1) \oplus \mathfrak{s o}(1,1)$ & \\
& $\mathfrak{s o}(2,7) \oplus \mathfrak{u}(1)$ & $\mathrm{R}$ \\
& $\mathfrak{s o}(9) \oplus \mathfrak{u}(1)$ & \\
\hline
\end{tabular}


Table 40: Maximal subalgebras of $\mathfrak{s o}(4,7)$.

\begin{tabular}{|l|r|l|}
\hline type & real subalgebras & \\
\hline \multirow{5}{*}{$A_{3}+B_{2}$} & $\mathfrak{s l}(4, \mathbb{R}) \oplus \mathfrak{s o}(4,1)$ & \\
& $\mathfrak{s u}(2,2) \oplus \mathfrak{s o}(2,3)$ & \\
& $\mathfrak{s l}(2, \mathbb{H}) \oplus \mathfrak{s o}(2,3)$ & $\mathrm{R}$ \\
& $\mathfrak{s u}(2,2) \oplus \mathfrak{s o}(5)$ & \\
& $\mathfrak{s u}(4) \oplus \mathfrak{s o}(4,1)$ & \\
\hline \multirow{3}{*}{$A_{1}+A_{1}+B_{3}$} & $\mathfrak{s u}(2) \oplus \mathfrak{s u}(2) \oplus \mathfrak{s o}(4,3)$ & $\mathrm{R}$ \\
& $\mathfrak{s l}) \oplus \mathfrak{s l}(2, \mathbb{R}) \oplus \mathfrak{s o}(2,5)$ & \\
& $\mathfrak{s u}(2) \oplus \mathfrak{s u}(2) \oplus \mathfrak{s o}(7)$ & \\
\hline & $\mathfrak{s u}(2) \oplus \mathfrak{s o}(4,4)$ & \\
$A_{1}+D_{4}$ & $\mathfrak{s l}(2, \mathbb{R}) \oplus \mathfrak{s o}(3,5)$ & $\mathrm{R}$ \\
& $\mathfrak{s l}(2, \mathbb{R}) \oplus \mathfrak{s o}(8)$ & \\
& $\mathfrak{s u}(2) \oplus \mathfrak{s o}(1,7)$ & \\
\hline$D_{5}$ & $\mathfrak{s o}(4,6)$ & $\mathrm{R}$ \\
\hline \multirow{3}{*}{$B_{4}+T_{1}$} & $\mathfrak{s o}(3,7)$ & $\mathrm{R}$ \\
& $\mathfrak{s o}(6,3) \oplus \mathfrak{s o}(1,1)$ & \\
\hline
\end{tabular}

Table 41: Maximal subalgebras of $\mathfrak{s o}(6,5)$.

\begin{tabular}{|l|r|l|}
\hline type & real subalgebras & \\
\hline & $\mathfrak{s l}(4, \mathbb{R}) \oplus \mathfrak{s o}(2,3)$ & \\
& $\mathfrak{s u}(2,2) \oplus \mathfrak{s o}(2,3)$ & \\
$A_{3}+B_{2}$ & $\mathfrak{s u}(2,2) \oplus \mathfrak{s o}(4,1)$ & \\
& $\mathfrak{s l}(2, \mathbb{H}) \oplus \mathfrak{s o}(4,1)$ & $\mathrm{R}$ \\
& $\mathfrak{s l}(2, \mathbb{H}) \oplus \mathfrak{s o}(5)$ & \\
& $\mathfrak{s u}(4) \oplus \mathfrak{s o}(5)$ & \\
\hline & $\mathfrak{s l}(2, \mathbb{C}) \oplus \mathfrak{s o}(4,3)$ & \\
$A_{1}+A_{1}+B_{3}$ & $\mathfrak{s l}(2, \mathbb{C}) \oplus \mathfrak{s o}(2,5)$ & $\mathrm{R}$ \\
& $\mathfrak{s u}(2) \oplus \mathfrak{s u}(2) \oplus \mathfrak{s o}(2,5)$ & \\
& $\mathfrak{s u}(2) \oplus \mathfrak{s u}(2) \oplus \mathfrak{s o}(6,1)$ & \\
\hline & $\mathfrak{s l}(2, \mathbb{R}) \oplus \mathfrak{s o}(4,4)$ & \\
& $\mathfrak{s l}(2, \mathbb{R}) \oplus \mathfrak{s o}(3,5)$ & $\mathrm{R}$ \\
$A_{1}+D_{4}$ & $\mathfrak{s u}(2) \oplus \mathfrak{s o}(3,5)$ & \\
& $\mathfrak{s u}(2) \oplus \mathfrak{s o}(8)$ & $\mathrm{R}$ \\
\hline \multirow{2}{*}{$D_{5}$} & $\mathfrak{s o}(5,5)$ & \\
& $\mathfrak{s o}(4,6)$ & \\
\hline
\end{tabular}




\begin{tabular}{|l|r|l|}
\hline \multicolumn{2}{|c|}{ subalgebras of $\mathfrak{s o}(6,5)$} \\
\hline \multirow{4}{*}{$B_{4}+T_{1}$} & $\mathfrak{s o}(4,5) \oplus \mathfrak{s o}(1,1)$ & \\
& $\mathfrak{s o}(4,5) \oplus \mathfrak{u}(1)$ & $\mathrm{R}$ \\
& $\mathfrak{s o}(6,3) \oplus \mathfrak{u}(1)$ & \\
\hline$A_{1}$ & $\mathfrak{s l}(2, \mathbb{R})$ & $\mathrm{S}$ \\
\hline
\end{tabular}

Table 42: Maximal subalgebras of $\mathfrak{s o}(8,3)$.

\begin{tabular}{|l|r|l|}
\hline type & real subalgebras & \\
\hline & $\mathfrak{s l}(4, \mathbb{R}) \oplus \mathfrak{s o}(5)$ & \\
$A_{3}+B_{2}$ & $\mathfrak{s u}(2,2) \oplus \mathfrak{s o}(4,1)$ & $\mathrm{R}$ \\
& $\mathfrak{s l}(2, \mathbb{H}) \oplus \mathfrak{s o}(2,3)$ & \\
& $\mathfrak{s u}(4) \oplus \mathfrak{s o}(2,3)$ & \\
\hline & $\mathfrak{s l}(2, \mathbb{R}) \oplus \mathfrak{s l}(2, \mathbb{R}) \oplus \mathfrak{s o}(6,1)$ & \\
$A_{1}+A_{1}+B_{3}$ & $\mathfrak{s l}(2, \mathbb{C}) \oplus \mathfrak{s o}(2,5)$ & $\mathrm{R}$ \\
& $\mathfrak{s u}(2) \oplus \mathfrak{s u}(2) \oplus \mathfrak{s o}(4,3)$ & \\
& $\mathfrak{s l}(2, \mathbb{C}) \oplus \mathfrak{s o}(7)$ & \\
\hline \multirow{3}{*}{$A_{1}+D_{4}$} & $\mathfrak{s l}(2) \oplus \mathbb{R}) \oplus \mathfrak{s o}(8)$ & \\
& $\mathfrak{s l}(2, \mathbb{R}) \oplus \mathfrak{s o}(1,7)$ & $\mathrm{R}$ \\
& $\mathfrak{s u}(2) \oplus \mathfrak{s o}(8)$ & \\
\hline \multirow{2}{*}{$D_{5}$} & $\mathfrak{s o}(3,7)$ & $\mathrm{R}$ \\
\hline \multirow{3}{*}{$B_{4}+T_{1}$} & $\mathfrak{s o}(2,8)$ & $\mathrm{R}$ \\
& $\mathfrak{s o}(2,7) \oplus \mathfrak{s o}(1,1)$ & \\
\hline
\end{tabular}

Table 43: Maximal subalgebras of $\mathfrak{s o}(10,1)$.

\begin{tabular}{|l|c|l|}
\hline type & real subalgebras & \\
\hline$A_{3}+B_{2}$ & $\begin{array}{c}\mathfrak{s l}(2, \mathbb{H}) \oplus \mathfrak{s o}(5) \\
\mathfrak{s u}(4) \oplus \mathfrak{s o}(4,1)\end{array}$ & $\mathrm{R}$ \\
\hline$A_{1}+A_{1}+B_{3}$ & $\mathfrak{s l}(2, \mathbb{C}) \oplus \mathfrak{s o}(7)$ & $\mathrm{R}$ \\
\hline$A_{1}+D_{4}$ & $\begin{array}{c}\mathfrak{s u}(2) \oplus \mathfrak{s u}(2) \oplus \mathfrak{s o}(6,1) \\
\mathfrak{s l}(2, \mathbb{R}) \oplus \mathfrak{s o}(8)\end{array}$ & $\mathrm{R}$ \\
\hline$D_{5}$ & $\begin{array}{c}\mathfrak{s o}(9,1) \\
\mathfrak{s o}(10)\end{array}$ & $\mathrm{R}$ \\
\hline$B_{4}+T_{1}$ & $\begin{array}{c}\mathfrak{s o}(9) \oplus \mathfrak{s o}(1,1) \\
\mathfrak{s o}(8,1) \oplus \mathfrak{u}(1)\end{array}$ & $\mathrm{R}$ \\
\hline
\end{tabular}

Type $C_{5}$

Table 44: Maximal subalgebras of $\mathfrak{s p}(1,4)$.

\begin{tabular}{|l|c|l|}
\hline type & real subalgebras & \\
\hline$A_{4}+T_{1}$ & $\mathfrak{s u}(1,4) \oplus \mathfrak{u}(1)$ & $\mathrm{R}$ \\
\hline
\end{tabular}




\begin{tabular}{|c|r|l|}
\hline \multicolumn{3}{|c|}{ subalgebras of $\mathfrak{s p}(1,4)$} \\
\hline$B_{2}+C_{3}$ & $\begin{array}{r}\mathfrak{s o}(4,1) \oplus \mathfrak{s p}(3) \\
\mathfrak{s o}(5) \oplus \mathfrak{s p}(1,2)\end{array}$ & $\mathrm{R}$ \\
\hline$A_{1}+C_{4}$ & $\mathfrak{s u}(2) \oplus \mathfrak{s p}(1,3)$ & \\
& $\mathfrak{s u}(2) \oplus \mathfrak{s p}(4)$ & $\mathrm{R}$ \\
\hline$A_{1}+B_{2}$ & $\mathfrak{s u}(2) \oplus \mathfrak{s o}(4,1)$ & $\mathrm{S}$ \\
\hline
\end{tabular}

Table 45: Maximal subalgebras of $\mathfrak{s p}(2,3)$.

\begin{tabular}{|l|r|l|}
\hline \multicolumn{1}{|l|}{ type } & real subalgebras & \\
\hline$A_{4}+T_{1}$ & $\mathfrak{s u}(2,3)$ & $\mathrm{R}$ \\
\hline & $\mathfrak{s o}(4,1) \oplus \mathfrak{s p}(1,2)$ & \\
$B_{2}+C_{3}$ & $\mathfrak{s o}(5) \oplus \mathfrak{s p}(1,2)$ & $\mathrm{R}$ \\
& $\mathfrak{s o}(5) \oplus \mathfrak{s p}(3)$ & \\
\hline$A_{1}+C_{4}$ & $\mathfrak{s u}(2) \oplus \mathfrak{s p}(2,2)$ & $\mathrm{R}$ \\
& $\mathfrak{s u}(2) \oplus \mathfrak{s p}(1,3)$ & \\
\hline$A_{1}+B_{2}$ & $\mathfrak{s u}(2) \oplus \mathfrak{s o}(2,3)$ & $\mathrm{S}$ \\
\hline
\end{tabular}

Table 46: Maximal subalgebras of $\mathfrak{s p}(5, \mathbb{R})$.

\begin{tabular}{|l|r|l|}
\hline type & real subalgebras & \\
\hline & $\mathfrak{s l}(5, \mathbb{R}) \oplus \mathfrak{s o}(1,1)$ & \\
& $\mathfrak{s u}(2,3) \oplus \mathfrak{u}(1)$ & \\
$A_{4}+T_{1}$ & $\mathfrak{s u}(1,4) \oplus \mathfrak{u}(1)$ & $\mathrm{R}$ \\
& $\mathfrak{s u}(5) \oplus \mathfrak{u}(1)$ & \\
& $\mathfrak{s o}(2,3) \oplus \mathfrak{s p}(3, \mathbb{R})$ & $\mathrm{R}$ \\
\hline$B_{2}+C_{3}$ & $\mathfrak{s o}(2, \mathbb{R}) \oplus \mathfrak{s p}(4, \mathbb{R})$ & $\mathrm{R}$ \\
\hline$A_{1}+C_{4}$ & $\mathfrak{s l}(2 \mathfrak{s l}(2, \mathbb{R}) \oplus \mathfrak{s o}(5)$ & \\
& $\mathfrak{s l}(2, \mathbb{R}) \oplus \mathfrak{s o}(2,3)$ & $\mathrm{S}$ \\
$A_{1}+B_{2}$ & $\mathfrak{s l}(2, \mathbb{R}) \oplus \mathfrak{s o}(4,1)$ & \\
& $\mathfrak{s l}(2, \mathbb{R})$ & $\mathrm{S}$ \\
\hline$A_{1}$ & \multicolumn{3}{|c}{}
\end{tabular}

Type $D_{5}$

Table 47: Maximal subalgebras of $\mathfrak{s o}(2,8)$.

\begin{tabular}{|l|r|l|}
\hline type & real subalgebras & \\
\hline$A_{4}+T_{1}$ & $\mathfrak{s u}(1,4) \oplus \mathfrak{u}(1)(2 \times)$ & $\mathrm{R}$ \\
\hline \multirow{3}{*}{$A_{1}+A_{1}+A_{3}$} & $\mathfrak{s l}(2, \mathbb{R}) \oplus \mathfrak{s l}(2, \mathbb{R}) \oplus \mathfrak{s u}(4)$ & \\
& $\mathfrak{s l}(2, \mathbb{C}) \oplus \mathfrak{s l}(2, \mathbb{H})$ & $\mathrm{R}$ \\
& $\mathfrak{s u}(2) \oplus \mathfrak{s u}(2) \oplus \mathfrak{s u}(2,2)$ & \\
\hline \multirow{3}{*}{$D_{4}+T_{1}$} & $\mathfrak{s o}(1,7) \oplus \mathfrak{s o}(1,1)$ & \\
& $\mathfrak{s o}(8) \oplus \mathfrak{u}(1)$ & $\mathrm{R}$ \\
& $\mathfrak{s o}(8) \oplus \mathfrak{u}(1)$ & \\
\hline \multirow{2}{*}{$B_{2}+B_{2}$} & $\mathfrak{s o}(5) \oplus \mathfrak{s o}(2,3)$ & $\mathrm{S}$ \\
& $\mathfrak{s o}(4,1) \oplus \mathfrak{s o}(4,1)$ & \\
\hline
\end{tabular}




\begin{tabular}{|l|r|l|}
\hline \multicolumn{2}{|c|}{ subalgebras of $\mathfrak{s o}(2,8)$} \\
\hline \multirow{3}{*}{$A_{1}+B_{3}$} & $\mathfrak{s u}(2) \oplus \mathfrak{s o}(2,5)$ & \\
& $\mathfrak{s l}(2, \mathbb{R}) \oplus \mathfrak{s o}(7)$ & $\mathrm{S}$ \\
& $\mathfrak{s l}(2, \mathbb{R}) \oplus \mathfrak{s o}(6,1)$ & \\
\hline \multirow{2}{*}{$B_{4}$} & $\mathfrak{s o}(2,7)$ & $\mathrm{S}$ \\
& $\mathfrak{s o}(8,1)$ & \\
\hline
\end{tabular}

Table 48: Maximal subalgebras of $\mathfrak{s o}(4,6)$.

\begin{tabular}{|c|c|c|}
\hline type & real subalgebras & \\
\hline$A_{4}+T_{1}$ & $\mathfrak{s u}(2,3) \oplus \mathfrak{u}(1)(2 \times)$ & $\mathrm{R}$ \\
\hline \multirow{5}{*}{$A_{1}+A_{1}+A_{3}$} & $\mathfrak{s l}(2, \mathbb{R}) \oplus \mathfrak{s l}(2, \mathbb{R}) \oplus \mathfrak{s u}(2,2)$ & \multirow{5}{*}{$\mathrm{R}$} \\
\hline & $\mathfrak{s l}(2, \mathbb{C}) \oplus \mathfrak{s l}(4, \mathbb{R})$ & \\
\hline & $\mathfrak{s l}(2, \mathbb{C}) \oplus \mathfrak{s l}(2, \mathbb{H})$ & \\
\hline & $\mathfrak{s u}(2) \oplus \mathfrak{s u}(2) \oplus \mathfrak{s u}(2,2)$ & \\
\hline & $\mathfrak{s u}(2) \oplus \mathfrak{s u}(2) \oplus \mathfrak{s u}(4)$ & \\
\hline \multirow{3}{*}{$D_{4}+T_{1}$} & $\mathfrak{s o}(3,5) \oplus \mathfrak{s o}(1,1)$ & \multirow{3}{*}{$\mathrm{R}$} \\
\hline & $\mathfrak{s o}(4,4) \oplus \mathfrak{u}(1)$ & \\
\hline & $\mathfrak{s o}^{*}(8) \oplus \mathfrak{u}(1)$ & \\
\hline \multirow{2}{*}{$B_{2}$} & $\mathfrak{s o}(2,3)$ & \multirow{2}{*}{$\mathrm{S}$} \\
\hline & $\mathfrak{s o}(4,1)$ & \\
\hline \multirow{3}{*}{$B_{2}+B_{2}$} & $\mathfrak{s o}(5) \oplus \mathfrak{s o}(4,1)$ & \multirow{3}{*}{$\mathrm{S}$} \\
\hline & $\mathfrak{s o}(2,3) \oplus \mathfrak{s o}(2,3)$ & \\
\hline & $\mathfrak{s o}(2,3) \oplus \mathfrak{s o}(4,1)$ & \\
\hline \multirow{4}{*}{$A_{1}+B_{3}$} & $\mathfrak{s u}(2) \oplus \mathfrak{s o}(4,3)$ & \multirow{4}{*}{$\mathrm{S}$} \\
\hline & $\mathfrak{s u}(2) \oplus \mathfrak{s o}(6,1)$ & \\
\hline & $\mathfrak{s l}(2, \mathbb{R}) \oplus \mathfrak{s o}(2,5)$ & \\
\hline & $\mathfrak{s l}(2, \mathbb{R}) \oplus \mathfrak{s o}(4,3)$ & \\
\hline \multirow{2}{*}{$B_{4}$} & $\mathfrak{s o}(4,5)$ & \multirow{2}{*}{$\mathrm{S}$} \\
\hline & $\mathfrak{s o}(6,3)$ & \\
\hline
\end{tabular}

Table 49: Maximal subalgebras of $\mathfrak{s o}^{*}(10)$.

\begin{tabular}{|l|c|l|}
\hline type & real subalgebras & \\
\hline & $\mathfrak{s u}(2,3) \oplus \mathfrak{u}(1)$ & \\
$A_{4}+T_{1}$ & $\mathfrak{s u}(1,4) \oplus \mathfrak{u}(1)$ & $\mathrm{R}$ \\
& $\mathfrak{s u}(5) \oplus \mathfrak{u}(1)$ & \\
\hline$A_{1}+A_{1}+A_{3}$ & $\mathfrak{s u}(2) \oplus \mathfrak{s l}(2, \mathbb{R}) \oplus \mathfrak{s u}(1,3)$ & $\mathrm{R}$ \\
\hline$D_{4}+T_{1}$ & $\mathfrak{s o}^{*}(8) \oplus \mathfrak{u}(1)$ & $\mathrm{R}$ \\
\hline
\end{tabular}

Table 50: Maximal subalgebras of $\mathfrak{s o}(9,1)$.

\begin{tabular}{|l|r|l|}
\hline type & real subalgebras & \\
\hline$A_{1}+A_{1}+A_{3}$ & $\mathfrak{s u}(2) \oplus \mathfrak{s u}(2) \oplus \mathfrak{s l}(2, \mathbb{H})$ & $\mathrm{R}$ \\
& $\mathfrak{s l}(2, \mathbb{C}) \oplus \mathfrak{s u}(4)$ & \\
\hline \multirow{2}{*}{$D_{4}+T_{1}$} & $\mathfrak{s o}(1,7) \oplus \mathfrak{u}(1)$ & $\mathrm{R}$ \\
& $\mathfrak{s o}(8) \oplus \mathfrak{s o}(1,1)$ & \\
\hline
\end{tabular}




\begin{tabular}{|l|c|c|}
\hline \multicolumn{2}{|l|}{ subalgebras of $\mathfrak{s o}(9,1)$} \\
\hline$B_{2}+B_{2}$ & $\mathfrak{s o}(5) \oplus \mathfrak{s o}(4,1)$ & $\mathrm{S}$ \\
\hline$A_{1}+B_{3}$ & $\begin{array}{r}\mathfrak{s u}(2) \oplus \mathfrak{s o}(6,1) \\
\mathfrak{s l}(2, \mathbb{R}) \oplus \mathfrak{s o}(7)\end{array}$ & $\mathrm{S}$ \\
\hline$B_{4}$ & $\begin{array}{r}\mathfrak{s o}(9) \\
\mathfrak{s o}(8,1)\end{array}$ & $\mathrm{S}$ \\
\hline
\end{tabular}

Table 51: Maximal subalgebras of $\mathfrak{s o}(3,7)$.

\begin{tabular}{|l|r|l|}
\hline type & real subalgebras & \\
\hline & $\mathfrak{s l}(2, \mathbb{R}) \oplus \mathfrak{s l}(2, \mathbb{R}) \oplus \mathfrak{s l}(2, \mathbb{H})$ & \\
$A_{1}+A_{1}+A_{3}$ & $\mathfrak{s l}(2, \mathbb{C}) \oplus \mathfrak{s u}(2,2)$ & \\
& $\mathfrak{s u}(2) \oplus \mathfrak{s u}(2) \oplus \mathfrak{s l}(4, \mathbb{R})$ & $\mathrm{R}$ \\
& $\mathfrak{s l}(2, \mathbb{C}) \oplus \mathfrak{s u}(4)$ & \\
\hline \multirow{2}{*}{$D_{4}+T_{1}$} & $\mathfrak{s o}(8) \oplus \mathfrak{s o}(1,1)$ & \\
& $\mathfrak{s o}(3,5) \oplus \mathfrak{u}(1)$ & $\mathrm{R}$ \\
\hline$B_{2}+B_{2}$ & $\mathfrak{s o}(1,7) \oplus \mathfrak{u}(1)$ & \\
\hline \multirow{2}{*}{$A_{1}+B_{3}$} & $\mathfrak{s o}(5) \oplus \mathfrak{s o}(2,3)$ & $\mathrm{S}$ \\
& $\mathfrak{s o}(2,3) \oplus \mathfrak{s o}(4,1)$ & \\
& $\mathfrak{s u}(2) \oplus \mathfrak{s o}(4,3) \oplus \mathfrak{s o}(7)$ & $\mathrm{S}$ \\
\hline$B_{4}$ & $\mathfrak{s l}(2, \mathbb{R}) \oplus \mathfrak{s o}(2,5)$ & \\
& $\mathfrak{s l}(2, \mathbb{R}) \oplus \mathfrak{s o}(6,1)$ & $\mathrm{S}$ \\
\hline
\end{tabular}

Table 52: Maximal subalgebras of $\mathfrak{s o}(5,5)$.

\begin{tabular}{|l|r|l|}
\hline type & real subalgebras & \\
\hline$A_{4}+T_{1}$ & $\mathfrak{s l}(5, \mathbb{R}) \oplus \mathfrak{s o}(1,1)$ & $\mathrm{R}$ \\
\hline \multirow{3}{*}{$A_{1}+A_{1}+A_{3}$} & $\mathfrak{s l}(2, \mathbb{R}) \oplus \mathfrak{s l}(2, \mathbb{R}) \oplus \mathfrak{s l}(4, \mathbb{R})$ & \\
& $\mathfrak{s l}(2, \mathbb{C}) \oplus \mathfrak{s u}(2,2)(2 \times)$ & $\mathrm{R}$ \\
& $\mathfrak{s u}(2) \oplus \mathfrak{s u}(2) \oplus \mathfrak{s l}(2, \mathbb{H})(2 \times)$ & \\
\hline$D_{4}+T_{1}$ & $\mathfrak{s o}(4,4) \oplus \mathfrak{s o}(1,1)$ & $\mathrm{R}$ \\
\hline & $\mathfrak{s o}(3,5) \oplus \mathfrak{u}(1)(2 \times)$ & \\
$B_{2}+B_{2}$ & $\mathfrak{s o}(5) \oplus \mathfrak{s o}(5)$ & \\
& $\mathfrak{s o}(4,1) \oplus \mathfrak{s o}(4,1)$ & \\
\hline \multirow{2}{*}{$A_{1}+B_{3}$} & $\mathfrak{s u}(2) \oplus \mathfrak{s o}(2,5)$ & $\mathrm{S}$ \\
\hline$B_{4}$ & $\mathfrak{s l}(2, \mathbb{R}) \oplus \mathfrak{s o}(4,3)$ & \\
\hline
\end{tabular}


Type $A_{6}$

Table 53: Maximal subalgebras of $\mathfrak{s u}(1,6)$.

\begin{tabular}{|l|c|l|}
\hline type & real subalgebras & \\
\hline$A_{2}+A_{3}+T_{1}$ & $\begin{array}{c}\mathfrak{s u}(1,2) \oplus \mathfrak{s u}(4) \oplus \mathfrak{u}(1) \\
\mathfrak{s u}(3) \oplus \mathfrak{s u}(1,3) \oplus \mathfrak{u}(1)\end{array}$ & $\mathrm{R}$ \\
\hline$A_{1}+A_{4}+T_{1}$ & $\begin{array}{c}\mathfrak{s u}(2) \oplus \mathfrak{s u}(1,4) \oplus \mathfrak{u}(1) \\
\mathfrak{s l}(2, \mathbb{R}) \oplus \mathfrak{s u}(5) \oplus \mathfrak{u}(1)\end{array}$ & $\mathrm{R}$ \\
\hline$A_{5}+T_{1}$ & $\mathfrak{s u}(1,5) \oplus \mathfrak{u}(1)$ & $\mathrm{R}$ \\
\hline$B_{3}$ & $\mathfrak{s u}(6) \oplus \mathfrak{u}(1)$ & \\
\hline
\end{tabular}

Table 54: Maximal subalgebras of $\mathfrak{s u}(2,5)$.

\begin{tabular}{|l|c|l|}
\hline type & real subalgebras & \\
\hline & $\mathfrak{s u}(1,2) \oplus \mathfrak{s u}(1,3) \oplus \mathfrak{u}(1)$ & \\
$A_{2}+A_{3}+T_{1}$ & $\mathfrak{s u}(3) \oplus \mathfrak{s u}(2,2) \oplus \mathfrak{u}(1)$ & $\mathrm{R}$ \\
& $\mathfrak{s u}(1,2) \oplus \mathfrak{s u}(4) \oplus \mathfrak{u}(1)$ & \\
\hline & $\mathfrak{s u}(2) \oplus \mathfrak{s u}(2,3) \oplus \mathfrak{u}(1)$ & \\
$A_{1}+A_{4}+T_{1}$ & $\mathfrak{s l}(2, \mathbb{R}) \oplus \mathfrak{s u}(1,4) \oplus \mathfrak{u}(1)$ & $\mathrm{R}$ \\
& $\mathfrak{s u}(2) \oplus \mathfrak{s u}(5) \oplus \mathfrak{u}(1)$ & \\
\hline \multirow{2}{*}{$A_{5}+T_{1}$} & $\mathfrak{s u}(2,4) \oplus \mathfrak{u}(1)$ & $\mathrm{R}$ \\
\hline$B_{3}$ & $\mathfrak{s u}(1,5) \oplus \mathfrak{u}(1)$ & \\
\hline
\end{tabular}

Table 55: Maximal subalgebras of $\mathfrak{s u}(3,4)$.

\begin{tabular}{|l|r|l|}
\hline type & \multicolumn{1}{|c|}{ real subalgebras } & \\
\hline & $\mathfrak{s u}(1,2) \oplus \mathfrak{s u}(2,2) \oplus \mathfrak{u}(1)$ & \\
$A_{2}+A_{3}+T_{1}$ & $\mathfrak{s u}(1,2) \oplus \mathfrak{s u}(1,3) \oplus \mathfrak{u}(1)$ & \\
& $\mathfrak{s u}(3) \oplus \mathfrak{s u}(1,3) \oplus \mathfrak{u}(1)$ & $\mathrm{R}$ \\
& $\mathfrak{s u}(3) \oplus \mathfrak{s u}(4) \oplus \mathfrak{u}(1)$ & \\
\hline & $\mathfrak{s l}(2, \mathbb{R}) \oplus \mathfrak{s u}(2,3) \oplus \mathfrak{u}(1)$ & \\
$A_{1}+A_{4}+T_{1}$ & $\mathfrak{s u}(2) \oplus \mathfrak{s u}(2,3) \oplus \mathfrak{u}(1)$ & $\mathrm{R}$ \\
& $\mathfrak{s u}(2) \oplus \mathfrak{s u}(1,4) \oplus \mathfrak{u}(1)$ & \\
\hline \multirow{2}{*}{$A_{5}+T_{1}$} & $\mathfrak{s u}(3,3) \oplus \mathfrak{u}(1)$ & $\mathrm{R}$ \\
\hline$B_{3}$ & $\mathfrak{s u}(2,4) \oplus \mathfrak{u}(1)$ & \\
\hline
\end{tabular}

Table 56: Maximal subalgebras of $\mathfrak{s l}(7, \mathbb{R})$.

\begin{tabular}{|l|c|c|}
\hline type & real subalgebras & \\
\hline$A_{2}+A_{3}+T_{1}$ & $\mathfrak{s l}(3, \mathbb{R}) \oplus \mathfrak{s l}(4, \mathbb{R}) \oplus \mathfrak{s o}(1,1)$ & $\mathrm{R}$ \\
\hline$A_{1}+A_{4}+T_{1}$ & $\mathfrak{s l}(2, \mathbb{R}) \oplus \mathfrak{s l}(5, \mathbb{R}) \oplus \mathfrak{s o}(1,1)$ & $\mathrm{R}$ \\
\hline$A_{5}+T_{1}$ & $\mathfrak{s l}(6, \mathbb{R}) \oplus \mathfrak{s o}(1,1)$ & $\mathrm{R}$ \\
\hline
\end{tabular}




\begin{tabular}{|l|r|l|}
\hline \multirow{3}{*}{$B_{3}$} & $\mathfrak{s o}(7)$ & \\
& $\mathfrak{s o}(2,5)$ & $\mathrm{S}$ \\
& $\mathfrak{s o}(4,3)$ & \\
& $\mathfrak{s o}(6,1)$ & \\
\hline
\end{tabular}

Type $B_{6}$

Table 57: Maximal subalgebras of $\mathfrak{s o}(2,11)$.

\begin{tabular}{|l|c|l|}
\hline type & real subalgebras & \\
\hline & $\mathfrak{s u}(2,2) \oplus \mathfrak{s o}(7)$ & \\
$A_{3}+B_{3}$ & $\mathfrak{s l}(2, \mathbb{H}) \oplus \mathfrak{s o}(6,1)$ & $\mathrm{R}$ \\
& $\mathfrak{s u}(4) \oplus \mathfrak{s o}(2,5)$ & \\
\hline & $\mathfrak{s o}(5) \oplus \mathfrak{s o}{ }^{*}(8)$ & \\
$B_{2}+D_{4}$ & $\mathfrak{s o}(4,1) \oplus \mathfrak{s o}(1,7)$ & $\mathrm{R}$ \\
& $\mathfrak{s o}(2,3) \oplus \mathfrak{s o}(8)$ & \\
\hline & $\mathfrak{s l}(2, \mathbb{R}) \oplus \mathfrak{s l}(2, \mathbb{R}) \oplus \mathfrak{s o}(9)$ & \\
$A_{1}+A_{1}+B_{4}$ & $\mathfrak{s l}(2, \mathbb{C}) \oplus \mathfrak{s o}(8,1)$ & $\mathrm{R}$ \\
& $\mathfrak{s u}(2) \oplus \mathfrak{s u}(2) \oplus \mathfrak{s o}(2,7)$ & \\
\hline & $\mathfrak{s u}(2) \oplus \mathfrak{s o}(2,8)$ & \\
$A_{1}+D_{5}$ & $\mathfrak{s l}(2, \mathbb{R}) \oplus \mathfrak{s o}(9,1)$ & $\mathrm{R}$ \\
& $\mathfrak{s l}(2, \mathbb{R}) \oplus \mathfrak{s o}(10)$ & \\
\hline \multirow{2}{*}{$D_{6}$} & $\mathfrak{s o}(2,10)$ & $\mathrm{R}$ \\
\hline \multirow{3}{*}{$B_{5}+T_{1}$} & $\mathfrak{s o}(11,1)$ & \\
& $\mathfrak{s o}(10,1) \oplus \mathfrak{s o}(1,1)$ & \\
& $\mathfrak{s o}(2,9) \oplus \mathfrak{u}(1)$ & $\mathrm{R}$ \\
& $\mathfrak{s o}(11) \oplus \mathfrak{u}(1)$ & \\
\hline
\end{tabular}

Table 58: Maximal subalgebras of $\mathfrak{s o}(4,9)$.

\begin{tabular}{|l|r|l|}
\hline type & real subalgebras & \\
\hline & $\mathfrak{s l}(4, \mathbb{R}) \oplus \mathfrak{s o}(6,1)$ & \\
& $\mathfrak{s u}(2,2) \oplus \mathfrak{s o}(2,5)$ & \\
$A_{3}+B_{3}$ & $\mathfrak{s l}(2, \mathbb{H}) \oplus \mathfrak{s o}(4,3)$ & $\mathrm{R}$ \\
& $\mathfrak{s u}(4) \oplus \mathfrak{s o}(4,3)$ & \\
& $\mathfrak{s u}(2,2) \oplus \mathfrak{s o}(7)$ & \\
\hline & $\mathfrak{s o}(5) \oplus \mathfrak{s o}(4,4)$ & \\
$B_{2}+D_{4}$ & $\mathfrak{s o}(4,1) \oplus \mathfrak{s o}(3,5)$ & \\
& $\mathfrak{s o}(2,3) \oplus \mathfrak{s o}{ }^{*}(8)$ & $\mathrm{R}$ \\
& $\mathfrak{s o}(2,3) \oplus \mathfrak{s o}(1,7)$ & \\
& $\mathfrak{s o}(4,1) \oplus \mathfrak{s o}(8)$ & \\
\hline & $\mathfrak{s l}(2, \mathbb{R}) \oplus \mathfrak{s l}(2, \mathbb{R}) \oplus \mathfrak{s o}(2,7)$ & \\
& $\mathfrak{s l}(2, \mathbb{C}) \oplus \mathfrak{s o}(6,3)$ & \\
$A_{1}+A_{1}+B_{4}$ & $\mathfrak{s u}(2) \oplus \mathfrak{s u}(2) \oplus \mathfrak{s o}(4,5)$ & $\mathrm{R}$ \\
& $\mathfrak{s l}(2, \mathbb{C}) \oplus \mathfrak{s o}(8,1)$ & \\
& $\mathfrak{s u}(2) \oplus \mathfrak{s u}(2) \oplus \mathfrak{s o}(9)$ & \\
\hline
\end{tabular}




\begin{tabular}{|l|r|l|}
\hline \multicolumn{2}{|c|}{ subalgebras of $\mathfrak{s o}(4,9)$} \\
\hline \multirow{5}{*}{$A_{1}+D_{5}$} & $\mathfrak{s u}(2) \oplus \mathfrak{s o}(4,6)$ & \\
& $\mathfrak{s l}(2, \mathbb{R}) \oplus \mathfrak{s o}(3,7)$ & $\mathrm{R}$ \\
& $\mathfrak{s l}(2, \mathbb{R}) \oplus \mathfrak{s o}(2,8)$ & \\
& $\mathfrak{s u}(2) \oplus \mathfrak{s o}(9,1)$ & \\
\hline$D_{6}$ & $\mathfrak{s o}(4,8)$ & $\mathrm{R}$ \\
\hline \multirow{3}{*}{$B_{5}+T_{1}$} & $\mathfrak{s o}(3,9)$ & \\
& $\mathfrak{s o}(8,3) \oplus \mathfrak{s o}(1,1)$ & $\mathrm{R}$ \\
& $\mathfrak{s o}(4,7) \oplus \mathfrak{u}(1)$ & \\
\hline
\end{tabular}

Table 59: Maximal subalgebras of $\mathfrak{s o}(6,7)$.

\begin{tabular}{|c|c|c|}
\hline type & real subalgebras & \\
\hline \multirow{7}{*}{$A_{3}+B_{3}$} & $\mathfrak{s l}(4, \mathbb{R}) \oplus \mathfrak{s o}(4,3)$ & \multirow{7}{*}{$\mathrm{R}$} \\
\hline & $\mathfrak{s u}(2,2) \oplus \mathfrak{s o}(4,3)$ & \\
\hline & $\mathfrak{s u}(2,2) \oplus \mathfrak{s o}(2,5)$ & \\
\hline & $\mathfrak{s l}(2, \mathbb{H}) \oplus \mathfrak{s o}(2,5)$ & \\
\hline & $\mathfrak{s l}(2, \mathbb{H}) \oplus \mathfrak{s o}(6,1)$ & \\
\hline & $\mathfrak{s u}(4) \oplus \mathfrak{s o}(6,1)$ & \\
\hline & $\mathfrak{s u}(4) \oplus \mathfrak{s o}(7)$ & \\
\hline \multirow{6}{*}{$B_{2}+D_{4}$} & $\mathfrak{s o}(2,3) \oplus \mathfrak{s o}(4,4)$ & \multirow{6}{*}{$\mathrm{R}$} \\
\hline & $\mathfrak{s o}(2,3) \oplus \mathfrak{s o}(3,5)$ & \\
\hline & $\mathfrak{s o}(4,1) \oplus \mathfrak{s o}(3,5)$ & \\
\hline & $\mathfrak{s o}(4,1) \oplus \mathfrak{s o}^{*}(8)$ & \\
\hline & $\mathfrak{s o}(5) \oplus \mathfrak{s o}^{*}(8)$ & \\
\hline & $\mathfrak{s o}(5) \oplus \mathfrak{s o}(1,7)$ & \\
\hline \multirow{5}{*}{$A_{1}+A_{1}+B_{4}$} & $\mathfrak{s l}(2, \mathbb{R}) \oplus \mathfrak{s l}(2, \mathbb{R}) \oplus \mathfrak{s o}(4,5)$ & \multirow{5}{*}{$\mathrm{R}$} \\
\hline & $\mathfrak{s l}(2, \mathbb{C}) \oplus \mathfrak{s o}(4,5)$ & \\
\hline & $\mathfrak{s l}(2, \mathbb{C}) \oplus \mathfrak{s o}(6,3)$ & \\
\hline & $\mathfrak{s u}(2) \oplus \mathfrak{s u}(2) \oplus \mathfrak{s o}(6,3)$ & \\
\hline & $\mathfrak{s u}(2) \oplus \mathfrak{s u}(2) \oplus \mathfrak{s o}(2,7)$ & \\
\hline \multirow{4}{*}{$A_{1}+D_{5}$} & $\mathfrak{s l}(2, \mathbb{R}) \oplus \mathfrak{s o}(5,5)$ & \multirow{4}{*}{$\mathrm{R}$} \\
\hline & $\mathfrak{s l}(2, \mathbb{R}) \oplus \mathfrak{s o}(4,6)$ & \\
\hline & $\mathfrak{s u}(2) \oplus \mathfrak{s o}(4,6)$ & \\
\hline & $\mathfrak{s u}(2) \oplus \mathfrak{s o}(3,7)$ & \\
\hline \multirow{2}{*}{$D_{6}$} & $\mathfrak{s o}(6,6)$ & \multirow{2}{*}{$\mathrm{R}$} \\
\hline & & \\
\hline \multirow{3}{*}{$B_{5}+T_{1}$} & $\mathfrak{s o}(6,5) \oplus \mathfrak{s o}(1,1)$ & \multirow{3}{*}{$\mathrm{R}$} \\
\hline & $\mathfrak{s o}(6,5) \oplus \mathfrak{u}(1)$ & \\
\hline & $\mathfrak{s o}(4,7) \oplus \mathfrak{u}(1)$ & \\
\hline$A_{1}$ & $\mathfrak{s l}(2, \mathbb{R})$ & $\mathrm{S}$ \\
\hline
\end{tabular}


Table 60: Maximal subalgebras of $\mathfrak{s o}(8,5)$.

\begin{tabular}{|c|c|c|}
\hline type & real subalgebras & \\
\hline \multirow{6}{*}{$A_{3}+B_{3}$} & $\mathfrak{s l}(4, \mathbb{R}) \oplus \mathfrak{s o}(2,5)$ & \multirow{6}{*}{$\mathrm{R}$} \\
\hline & $\mathfrak{s u}(2,2) \oplus \mathfrak{s o}(4,3)$ & \\
\hline & $\mathfrak{s l}(2, \mathbb{H}) \oplus \mathfrak{s o}(4,3)$ & \\
\hline & $\mathfrak{s u}(2,2) \oplus \mathfrak{s o}(6,1)$ & \\
\hline & $\mathfrak{s u}(4) \oplus \mathfrak{s o}(2,5)$ & \\
\hline & $\mathfrak{s l}(2, \mathbb{H}) \oplus \mathfrak{s o}(7)$ & \\
\hline \multirow{6}{*}{$B_{2}+D_{4}$} & $\mathfrak{s o}(4,1) \oplus \mathfrak{s o}(4,4)$ & \multirow{6}{*}{$\mathrm{R}$} \\
\hline & $\mathfrak{s o}(2,3) \oplus \mathfrak{s o}(3,5)$ & \\
\hline & $\mathfrak{s o}(2,3) \oplus \mathfrak{s o}^{*}(8)$ & \\
\hline & $\mathfrak{s o}(5) \oplus \mathfrak{s o}(3,5)$ & \\
\hline & $\mathfrak{s o}(4,1) \oplus \mathfrak{s o}(1,7)$ & \\
\hline & $\mathfrak{s o}(5) \oplus \mathfrak{s o}(8)$ & \\
\hline \multirow{5}{*}{$A_{1}+A_{1}+B_{4}$} & $\mathfrak{s l}(2, \mathbb{R}) \oplus \mathfrak{s l}(2, \mathbb{R}) \oplus \mathfrak{s o}(6,3)$ & \multirow{5}{*}{$\mathrm{R}$} \\
\hline & $\mathfrak{s l}(2, \mathbb{C}) \oplus \mathfrak{s o}(4,5)$ & \\
\hline & $\mathfrak{s u}(2) \oplus \mathfrak{s u}(2) \oplus \mathfrak{s o}(4,5)$ & \\
\hline & $\mathfrak{s l}(2, \mathbb{C}) \oplus \mathfrak{s o}(2,7)$ & \\
\hline & $\mathfrak{s u}(2) \oplus \mathfrak{s u}(2) \oplus \mathfrak{s o}(8,1)$ & \\
\hline \multirow{4}{*}{$A_{1}+D_{5}$} & $\mathfrak{s u}(2) \oplus \mathfrak{s o}(5,5)$ & \multirow{4}{*}{$\mathrm{R}$} \\
\hline & $\mathfrak{s l}(2, \mathbb{R}) \oplus \mathfrak{s o}(4,6)$ & \\
\hline & $\mathfrak{s l}(2, \mathbb{R}) \oplus \mathfrak{s o}(3,7)$ & \\
\hline & $\mathfrak{s u}(2) \oplus \mathfrak{s o}(2,8)$ & \\
\hline \multirow{2}{*}{$D_{6}$} & $\mathfrak{s o}(5,7)$ & \multirow{2}{*}{$\mathrm{R}$} \\
\hline & $\mathfrak{s o}(4,8)$ & \\
\hline \multirow{3}{*}{$B_{5}+T_{1}$} & $\mathfrak{s o}(4,7) \oplus \mathfrak{s o}(1,1)$ & \multirow{3}{*}{$\mathrm{R}$} \\
\hline & $\mathfrak{s o}(6,5) \oplus \mathfrak{u}(1)$ & \\
\hline & $\mathfrak{s o}(8,3) \oplus \mathfrak{u}(1)$ & \\
\hline
\end{tabular}

Table 61: Maximal subalgebras of $\mathfrak{s o}(10,3)$.

\begin{tabular}{|l|r|l|}
\hline type & real subalgebras & \\
\hline \multirow{3}{*}{$A_{3}+B_{3}$} & $\mathfrak{s l}(4, \mathbb{R}) \oplus \mathfrak{s o}(7)$ & \\
& $\mathfrak{s u}(2,2) \oplus \mathfrak{s o}(6,1)$ & $\mathrm{R}$ \\
& $\mathfrak{s l}(2, \mathbb{H}) \oplus \mathfrak{s o}(2,5)$ & \\
& $\mathfrak{s u}(4) \oplus \mathfrak{s o}(4,3)$ & \\
\hline \multirow{3}{*}{$B_{2}+D_{4}$} & $\mathfrak{s o}(5) \oplus \mathfrak{s o}(3,5)$ & \\
& $\mathfrak{s o}(4,1) \oplus \mathfrak{s o}(8)$ & $\mathrm{R}$ \\
& $\mathfrak{s o}(2,3) \oplus \mathfrak{s o}(1,7)$ & \\
& $\mathfrak{s o}(2,3) \oplus \mathfrak{s o}(8)$ & \\
\hline$A_{1}+A_{1}+B_{4}$ & $\mathfrak{s l}(2, \mathbb{R}) \oplus \mathfrak{s l}(2, \mathbb{R}) \oplus \mathfrak{s o}(8,1)$ & \\
& $\mathfrak{s l}(2, \mathbb{C}) \oplus \mathfrak{s o}(2,7)$ & $\mathrm{R}$ \\
& $\mathfrak{s u}(2) \oplus \mathfrak{s u}(2) \oplus \mathfrak{s o}(6,3)$ & \\
& $\mathfrak{s l}(2, \mathbb{C}) \oplus \mathfrak{s o}(9)$ & \\
\hline
\end{tabular}




\begin{tabular}{|l|r|l|}
\hline \multicolumn{2}{|c|}{ subalgebras of $\mathfrak{s o}(10,3)$} \\
\hline \multirow{5}{*}{$A_{1}+D_{5}$} & $\mathfrak{s u}(2) \oplus \mathfrak{s o}(3,7)$ & \\
& $\mathfrak{s l}(2, \mathbb{R}) \oplus \mathfrak{s o}(2,8)$ & $\mathrm{R}$ \\
& $\mathfrak{s l}(2, \mathbb{R}) \oplus \mathfrak{s o}(9,1)$ & \\
\hline \multirow{2}{*}{$D_{6}$} & $\mathfrak{s u}(2) \oplus \mathfrak{s o}(10)$ & \\
\hline \multirow{3}{*}{$B_{5}+T_{1}$} & $\mathfrak{s o}(3,9)$ & $\mathrm{R}$ \\
& $\mathfrak{s o}(2,10)$ & \\
& $\mathfrak{s o}(2,9) \oplus \mathfrak{s o}(1,1)$ & $\mathrm{R}$ \\
\hline
\end{tabular}

Table 62: Maximal subalgebras of $\mathfrak{s o}(12,1)$.

\begin{tabular}{|c|c|c|}
\hline type & real subalgebras & \\
\hline \multirow{2}{*}{$A_{3}+B_{3}$} & $\mathfrak{s l}(2, \mathbb{H}) \oplus \mathfrak{s o}(7)$ & $D$ \\
\hline & $\mathfrak{s u}(4) \oplus \mathfrak{s o}(6,1)$ & $\boldsymbol{K}$ \\
\hline \multirow{2}{*}{$B_{2}+D_{4}$} & $\mathfrak{s o}(5) \oplus \mathfrak{s o}(1,7)$ & $\mathrm{P}$ \\
\hline & $\mathfrak{s o}(4,1) \oplus \mathfrak{s o}(8)$ & $\kappa$ \\
\hline \multirow{2}{*}{$A_{1}+A_{1}+B_{4}$} & $\mathfrak{s l}(2, \mathbb{C}) \oplus \mathfrak{s o}(9)$ & $\mathrm{P}$ \\
\hline & $\mathfrak{s u}(2) \oplus \mathfrak{s u}(2) \oplus \mathfrak{s o}(8,1)$ & $\Lambda$ \\
\hline \multirow{2}{*}{$A_{1}+D_{5}$} & $\mathfrak{s u}(2) \oplus \mathfrak{s o}(9,1)$ & \\
\hline & $\mathfrak{s l}(2, \mathbb{R}) \oplus \mathfrak{s o}(10)$ & $\mathrm{R}$ \\
\hline \multirow{2}{*}{$D_{6}$} & $\mathfrak{s o}(11,1)$ & \\
\hline & $\mathfrak{s o}(12)$ & $\mathrm{R}$ \\
\hline \multirow{2}{*}{$B_{5}+T_{1}$} & $\mathfrak{s o}(11) \oplus \mathfrak{s o}(1,1)$ & \\
\hline & & $\mathrm{R}$ \\
\hline
\end{tabular}

Type $C_{6}$

Table 63: Maximal subalgebras of $\mathfrak{s p}(1,5)$.

\begin{tabular}{|l|c|c|}
\hline type & real subalgebras & \\
\hline$A_{5}+T_{1}$ & $\mathfrak{s u}(1,5) \oplus \mathfrak{u}(1)$ & $\mathrm{R}$ \\
\hline$C_{3}+C_{3}$ & $\mathfrak{s p}(3) \oplus \mathfrak{s p}(1,2)$ & $\mathrm{R}$ \\
\hline$B_{2}+C_{4}$ & $\mathfrak{s o}(4,1) \oplus \mathfrak{s p}(4)$ & $\mathrm{R}$ \\
& $\mathfrak{s o}(5) \oplus \mathfrak{s p}(1,3)$ & \\
\hline$A_{1}+C_{5}$ & $\mathfrak{s u}(2) \oplus \mathfrak{s p}(1,4)$ & $\mathrm{R}$ \\
& $\mathfrak{s u}(2) \oplus \mathfrak{s p}(5)$ & \\
\hline$A_{1}+A_{3}$ & $\mathfrak{s u}(2) \oplus \mathfrak{s l}(2, \mathbb{H})$ & $\mathrm{S}$ \\
\hline
\end{tabular}

Table 64: Maximal subalgebras of $\mathfrak{s p}(2,4)$.

\begin{tabular}{|l|c|c|}
\hline type & real subalgebras & \\
\hline$A_{5}+T_{1}$ & $\mathfrak{s u}(2,4) \oplus \mathfrak{u}(1)$ & $\mathrm{R}$ \\
\hline$C_{3}+C_{3}$ & $\mathfrak{s p}(1,2) \oplus \mathfrak{s p}(1,2)$ & \\
& $\mathfrak{s p}(3) \oplus \mathfrak{s p}(1,2)$ & $\mathrm{R}$ \\
\hline
\end{tabular}




\begin{tabular}{|r|r|r|}
\hline \multicolumn{3}{|c|}{ subalgebras of $\mathfrak{s p}(2,4)$} \\
\hline & $\mathfrak{s o}(4,1) \oplus \mathfrak{s p}(1,3)$ & \\
$B_{2}+C_{4}$ & $\mathfrak{s o}(5) \oplus \mathfrak{s p}(2,2)$ & $\mathrm{R}$ \\
& $\mathfrak{s o}(5) \oplus \mathfrak{s p}(4)$ & \\
\hline$A_{1}+C_{5}$ & $\mathfrak{s u}(2) \oplus \mathfrak{s p}(2,3)$ & \\
& $\mathfrak{s u}(2) \oplus \mathfrak{s p}(1,4)$ & $\mathrm{R}$ \\
\hline$A_{1}+B_{2}$ & $\mathfrak{s l}(2, \mathbb{R}) \oplus \mathfrak{s o}(5)$ & $\mathrm{S}$ \\
\hline$A_{1}+A_{3}$ & $\mathfrak{s u}(2) \oplus \mathfrak{s u}(2,2)$ & $\mathrm{S}$ \\
\hline
\end{tabular}

Table 65: Maximal subalgebras of $\mathfrak{s p}(3,3)$.

\begin{tabular}{|c|c|c|}
\hline type & real subalgebras & \\
\hline \multirow{2}{*}{$A_{5}+T_{1}$} & $\mathfrak{s l}(3, \mathbb{H}) \oplus \mathfrak{s o}(1,1)$ & \\
\hline & $\mathfrak{s u}(3,3) \oplus \mathfrak{u}(1)$ & $\mathrm{R}$ \\
\hline \multirow{3}{*}{$C_{3}+C_{3}$} & $\mathfrak{s p}(3, \mathbb{C})$ & \\
\hline & $\mathfrak{s p}(1,2) \oplus \mathfrak{s p}(1,2)$ & $\mathrm{R}$ \\
\hline & $\mathfrak{s p}(3) \oplus \mathfrak{s p}(3)$ & \\
\hline \multirow{2}{*}{$B_{2}+C_{4}$} & $\mathfrak{s o}(4,1) \oplus \mathfrak{s p}(2,2)$ & \multirow{2}{*}{$\mathrm{R}$} \\
\hline & $\mathfrak{s o}(5) \oplus \mathfrak{s p}(1,3)(2 \times)$ & \\
\hline$A_{1}+C_{5}$ & $\mathfrak{s u}(2) \oplus \mathfrak{s p}(2,3)(2 \times)$ & $\mathrm{R}$ \\
\hline$A_{1}+B_{2}$ & $\mathfrak{s u}(2)+\mathfrak{s o}(4,1)$ & $\mathrm{S}$ \\
\hline \multirow{2}{*}{$A_{1}+A_{3}$} & $\mathfrak{s u}(2) \oplus \mathfrak{s l}(4, \mathbb{R})$ & \multirow{2}{*}{$\mathrm{S}$} \\
\hline & $\mathfrak{s l}(2, \mathbb{R}) \oplus \mathfrak{s u}(1,3)$ & \\
\hline
\end{tabular}

Table 66: Maximal subalgebras of $\mathfrak{s p}(6, \mathbb{R})$.

\begin{tabular}{|l|r|l|}
\hline type & real subalgebras & \\
\hline & $\mathfrak{s l}(6, \mathbb{R}) \oplus \mathfrak{s o}(1,1)$ & \\
& $\mathfrak{s u}(3,3) \oplus \mathfrak{u}(1)$ & \\
$A_{5}+T_{1}$ & $\mathfrak{s u}(2,4) \oplus \mathfrak{u}(1)$ & $\mathrm{R}$ \\
& $\mathfrak{s u}(1,5) \oplus \mathfrak{u}(1)$ & \\
& $\mathfrak{s u}(6) \oplus \mathfrak{u}(1)$ & \\
\hline$C_{3}+C_{3}$ & $\mathfrak{s p}(3, \mathbb{R}) \oplus \mathfrak{s p}(3, \mathbb{R})$ & $\mathrm{R}$ \\
& $\mathfrak{s p}(3, \mathbb{C})$ & \\
\hline$B_{2}+C_{4}$ & $\mathfrak{s o}(2,3) \oplus \mathfrak{s p}(4, \mathbb{R})$ & $\mathrm{R}$ \\
\hline$A_{1}+C_{5}$ & $\mathfrak{s l}(2, \mathbb{R}) \oplus \mathfrak{s p}(5, \mathbb{R})$ & $\mathrm{R}$ \\
\hline$A_{1}$ & $\mathfrak{s l}(2, \mathbb{R})$ & $\mathrm{S}$ \\
\hline \multirow{2}{*}{$A_{1}+B_{2}$} & $\mathfrak{s u}(2) \oplus \mathfrak{s o}(2,3)$ & $\mathrm{S}$ \\
& $\mathfrak{s l}(2, \mathbb{R}) \oplus \mathfrak{s o}(2,3)$ & \\
\hline & $\mathfrak{s u}(2) \oplus \mathfrak{s u}(1,3)$ & \\
& $\mathfrak{s l}(2, \mathbb{R}) \oplus \mathfrak{s u}(4)$ & \\
$A_{1}+A_{3}$ & $\mathfrak{s l}(2, \mathbb{R}) \oplus \mathfrak{s u}(2,2)$ & $\mathrm{S}$ \\
& $\mathfrak{s l}(2, \mathbb{R}) \oplus \mathfrak{s l}(2, \mathbb{H})$ & \\
& $\mathfrak{s l}(2, \mathbb{R}) \oplus \mathfrak{s l}(4, \mathbb{R})$ & \\
\hline
\end{tabular}


Type $D_{6}$

Table 67: Maximal subalgebras of $\mathfrak{s o}(2,10)$.

\begin{tabular}{|l|r|l|}
\hline type & real subalgebras & \\
\hline$A_{5}+T_{1}$ & $\mathfrak{s u}(1,5) \oplus \mathfrak{u}(1)(2 \times)$ & $\mathrm{R}$ \\
\hline$A_{3}+A_{3}$ & $\mathfrak{s u}(4) \oplus \mathfrak{s u}(2,2)$ & $\mathrm{R}$ \\
& $\mathfrak{s l}(2, \mathbb{H}) \oplus \mathfrak{s l}(2, \mathbb{H})$ & \\
\hline & $\mathfrak{s l}(2, \mathbb{R}) \oplus \mathfrak{s l}(2, \mathbb{R}) \oplus \mathfrak{s o}(8)$ & \\
$A_{1}+A_{1}+D_{4}$ & $\mathfrak{s l}(2, \mathbb{C}) \oplus \mathfrak{s o}(1,7)$ & $\mathrm{R}$ \\
& $\mathfrak{s u}(2) \oplus \mathfrak{s u}(2) \oplus \mathfrak{s o}(8)$ & \\
\hline & $\mathfrak{s o}(9,1) \oplus \mathfrak{s o}(1,1)$ & \\
$D_{5}+T_{1}$ & $\mathfrak{s o}(2,8) \oplus \mathfrak{u}(1)$ & $\mathrm{R}$ \\
& $\mathfrak{s o}(10) \oplus \mathfrak{u}(1)$ & \\
\hline & $\mathfrak{s o}(5) \oplus \mathfrak{s o}(2,5)$ & \\
$B_{2}+B_{3}$ & $\mathfrak{s o}(2,3) \oplus \mathfrak{s o}(7)$ & $\mathrm{S}$ \\
& $\mathfrak{s o}(4,1) \oplus \mathfrak{s o}(6,1)$ & \\
\hline \multirow{3}{*}{$A_{1}+B_{4}$} & $\mathfrak{s u}(2) \oplus \mathfrak{s o}(2,7)$ & \\
& $\mathfrak{s l}(2, \mathbb{R}) \oplus \mathfrak{s o}(9)$ & $\mathrm{S}$ \\
\hline$B_{5}$ & $\mathfrak{s l}(2, \mathbb{R}) \oplus \mathfrak{s o}(8,1)$ & \\
\hline & $\mathfrak{s o}(2,9)$ & $\mathrm{S}$ \\
\hline
\end{tabular}

Table 68: Maximal subalgebras of $\mathfrak{s o}(4,8)$.

\begin{tabular}{|l|r|r|}
\hline type & real subalgebras & \\
\hline$A_{5}+T_{1}$ & $\mathfrak{s u}(2,4) \oplus \mathfrak{u}(1)(4 \times)$ & $\mathrm{R}$ \\
\hline \multirow{3}{*}{$A_{3}+A_{3}$} & $\mathfrak{s l}(2, \mathbb{H}) \oplus \mathfrak{s l}(4, \mathbb{R})$ & \\
& $\mathfrak{s u}(2,2) \oplus \mathfrak{s u}(2,2)$ & $\mathrm{R}$ \\
& $\mathfrak{s u}(4) \oplus \mathfrak{s u}(2,2)$ & \\
\hline & $\mathfrak{s l}(2, \mathbb{R}) \oplus \mathfrak{s l}(2, \mathbb{R}) \oplus \mathfrak{s o}{ }^{*}(8)$ & \\
& $\mathfrak{s l}(2, \mathbb{C}) \oplus \mathfrak{s o}(3,5)$ & \\
$A_{1}+A_{1}+D_{4}$ & $\mathfrak{s u}(2) \oplus \mathfrak{s u}(2) \oplus \mathfrak{s o}(4,4)$ & $\mathrm{R}$ \\
& $\mathfrak{s l}(2, \mathbb{C}) \oplus \mathfrak{s o}(1,7)$ & \\
& $\mathfrak{s u}(2) \oplus \mathfrak{s u}(2) \oplus \mathfrak{s o}(8)$ & \\
\hline \multirow{3}{*}{$D_{5}+T_{1}$} & $\mathfrak{s o}(3,7) \oplus \mathfrak{s o}(1,1)$ & \\
& $\mathfrak{s o}(4,6) \oplus \mathfrak{u}(1)$ & $\mathrm{R}$ \\
& $\mathfrak{s o}(2,8) \oplus \mathfrak{u}(1)$ & \\
\hline$A_{1}+C_{3}$ & $\mathfrak{s u}(2) \oplus \mathfrak{s p}(1,2)$ & $\mathrm{S}$ \\
\hline & $\mathfrak{s o}(5) \oplus \mathfrak{s o}(4,3)$ & \\
& $\mathfrak{s o}(2,3) \oplus \mathfrak{s o}(2,5)$ & \\
$B_{2}+B_{3}$ & $\mathfrak{s o}(2,3) \oplus \mathfrak{s o}(6,1)$ & $\mathrm{S}$ \\
& $\mathfrak{s o}(4,1) \oplus \mathfrak{s o}(7)$ & \\
& $\mathfrak{s o}(4,1) \oplus \mathfrak{s o}(4,3)$ & \\
\hline
\end{tabular}




\begin{tabular}{|l|r|l|}
\hline \multicolumn{2}{|c|}{ subalgebras of $\mathfrak{s o}(4,8)$} \\
\hline \multirow{3}{*}{$A_{1}+B_{4}$} & $\mathfrak{s u}(2) \oplus \mathfrak{s o}(4,5)$ & \\
& $\mathfrak{s u}(2) \oplus \mathfrak{s o}(8,1)$ & $\mathrm{S}$ \\
& $\mathfrak{s l}(2, \mathbb{R}) \oplus \mathfrak{s o}(2,7)$ & \\
& $\mathfrak{s l}(2, \mathbb{R}) \oplus \mathfrak{s o}(6,3)$ & \\
\hline \multirow{2}{*}{$B_{5}$} & $\mathfrak{s o}(4,7)$ & $\mathrm{S}$ \\
& $\mathfrak{s o}(8,3)$ & \\
\hline
\end{tabular}

Table 69: Maximal subalgebras of $\mathfrak{s o}(6,6)$.

\begin{tabular}{|l|c|c|}
\hline type & real subalgebras & \\
\hline$A_{5}+T_{1}$ & $\mathfrak{s l}(6, \mathbb{R}) \oplus \mathfrak{s o}(1,1)(2 \times)$ & $\mathrm{R}$ \\
\hline & $\mathfrak{s u}(3,3) \oplus \mathfrak{u}(1)(2 \times)$ & \\
& $\mathfrak{s l}(4, \mathbb{R}) \oplus \mathfrak{s l}(4, \mathbb{R})$ & \\
$A_{3}+A_{3}$ & $\mathfrak{s u}(2,2) \oplus \mathfrak{s u}(2,2)$ & \\
& $\mathfrak{s l}(4, \mathbb{C})(2 \times)$ & $\mathrm{R}$ \\
& $\mathfrak{s l}(2, \mathbb{H}) \oplus \mathfrak{s l}(2, \mathbb{H})$ & \\
& $\mathfrak{s u}(4) \oplus \mathfrak{s u}(4)$ & \\
\hline & $\mathfrak{s l}(2, \mathbb{R}) \oplus \mathfrak{s l}(2, \mathbb{R}) \oplus \mathfrak{s o}(4,4)$ & \\
$A_{1}+A_{1}+D_{4}$ & $\mathfrak{s l}(2, \mathbb{C}) \oplus \mathfrak{s o}(3,5)(2 \times)$ & $\mathrm{R}$ \\
& $\mathfrak{s u}(2) \oplus \mathfrak{s u}(2) \oplus \mathfrak{s o}(8)(2 \times)$ & \\
\hline$D_{5}+T_{1}$ & $\mathfrak{s o}(5,5) \oplus \mathfrak{s o}(1,1)$ & $\mathrm{R}$ \\
\hline$A_{1}+C_{3}$ & $\mathfrak{s o}(4,6) \oplus \mathfrak{u}(1)(2 \times)$ & \\
\hline & $\mathfrak{s l}(2, \mathbb{R}) \oplus \mathfrak{s p}(3, \mathbb{R})$ & $\mathrm{S}$ \\
\hline$B_{2}+B_{3}$ & $\mathfrak{s o}(5) \oplus \mathfrak{s o}(6,1)$ & \\
& $\mathfrak{s o}(2,3) \oplus \mathfrak{s o}(4,3)$ & $\mathrm{S}$ \\
\hline$A_{1}+B_{4}$ & $\mathfrak{s o}(4,1) \oplus \mathfrak{s o}(2,5)$ & \\
\hline$B_{5}$ & $\mathfrak{s u}(2) \oplus \mathfrak{s o}(6,3)$ & $\mathrm{S}$ \\
\hline & $\mathfrak{s l}(2, \mathbb{R}) \oplus \mathfrak{s o}(4,5)$ & $\mathrm{S}$ \\
\hline
\end{tabular}

Table 70: Maximal subalgebras of $\mathfrak{s o}^{*}(12)$.

\begin{tabular}{|l|r|l|}
\hline type & real subalgebras & \\
\hline & $\mathfrak{s u}(3,3) \oplus \mathfrak{u}(1)$ & \\
& $\mathfrak{s l}(3, \mathbb{H}) \oplus \mathfrak{s o}(1,1)$ & \\
$A_{5}+T_{1}$ & $\mathfrak{s u}(2,4) \oplus \mathfrak{u}(1)$ & $\mathrm{R}$ \\
& $\mathfrak{s u}(1,5) \oplus \mathfrak{u}(1)$ & \\
& $\mathfrak{s u}(6) \oplus \mathfrak{u}(1)$ & \\
\hline$A_{3}+A_{3}$ & $\mathfrak{s l}(4, \mathbb{C})$ & $\mathrm{R}$ \\
& $\mathfrak{s u}(1,3) \oplus \mathfrak{s u}(1,3)$ & \\
\hline$A_{1}+A_{1}+D_{4}$ & $\mathfrak{s u}(2) \oplus \mathfrak{s l}(2, \mathbb{R}) \oplus \mathfrak{s o}(8)$ & $\mathrm{R}$ \\
\hline$D_{5}+T_{1}$ & $\mathfrak{s o}(10) \oplus \mathfrak{u}(1)$ & $\mathrm{R}$ \\
\hline & $\mathfrak{s u}(2) \oplus \mathfrak{s p}(3, \mathbb{R})$ & \\
$A_{1}+C_{3}$ & $\mathfrak{s l}(2, \mathbb{R}) \oplus \mathfrak{s p}(3)$ & $\mathrm{S}$ \\
& $\mathfrak{s l}(2, \mathbb{R}) \oplus \mathfrak{s p}(1,2)$ & \\
\hline
\end{tabular}


Table 71: Maximal subalgebras of $\mathfrak{s o}(11,1)$.

\begin{tabular}{|l|c|l|}
\hline type & real subalgebras & \\
\hline$A_{3}+A_{3}$ & $\mathfrak{s u}(4) \oplus \mathfrak{s l}(2, \mathbb{H})$ & $\mathrm{R}$ \\
\hline$A_{1}+A_{1}+D_{4}$ & $\mathfrak{s l}(2, \mathbb{C}) \oplus \mathfrak{s o}(8)$ & $\mathrm{R}$ \\
\hline$D_{5}+T_{1}$ & $\begin{array}{c}\mathfrak{s o}(2) \oplus \mathfrak{s u}(2) \oplus \mathfrak{s o}(1,7) \oplus \mathfrak{u}(1) \\
\mathfrak{s o}(10) \oplus \mathfrak{s o}(1,1)\end{array}$ & $\mathrm{R}$ \\
\hline$B_{2}+B_{3}$ & $\begin{array}{c}\mathfrak{s o}(5) \oplus \mathfrak{s o}(6,1) \\
\mathfrak{s o}(4,1) \oplus \mathfrak{s o}(7)\end{array}$ & $\mathrm{S}$ \\
\hline$A_{1}+B_{4}$ & $\begin{array}{c}\mathfrak{s u}(2) \oplus \mathfrak{s o}(8,1) \\
\mathfrak{s l}(2, \mathbb{R}) \oplus \mathfrak{s o}(9)\end{array}$ & $\mathrm{S}$ \\
\hline$B_{5}$ & $\mathfrak{s o}(11)$ & $\mathrm{S}$ \\
\hline
\end{tabular}

Table 72: Maximal subalgebras of $\mathfrak{s o}(3,9)$.

\begin{tabular}{|c|c|c|}
\hline type & real subalgebras & \\
\hline \multirow{2}{*}{$A_{3}+A_{3}$} & $\mathfrak{s u}(4) \oplus \mathfrak{s l}(4, \mathbb{R})$ & \multirow{2}{*}{$\mathrm{R}$} \\
\hline & $\mathfrak{s u}(2,2) \oplus \mathfrak{s l}(2, \mathbb{H})$ & \\
\hline \multirow{4}{*}{$A_{1}+A_{1}+D_{4}$} & $\mathfrak{s l}(2, \mathbb{R}) \oplus \mathfrak{s l}(2, \mathbb{R}) \oplus \mathfrak{s o}(1,7)$ & \multirow{4}{*}{$\mathrm{R}$} \\
\hline & $\mathfrak{s l}(2, \mathbb{C}) \oplus \mathfrak{s o}^{*}(8)$ & \\
\hline & $\mathfrak{s u}(2) \oplus \mathfrak{s u}(2) \oplus \mathfrak{s o}(3,5)$ & \\
\hline & $\mathfrak{s l}(2, \mathbb{C}) \oplus \mathfrak{s o}(8)$ & \\
\hline \multirow{3}{*}{$D_{5}+T_{1}$} & $\mathfrak{s o}(2,8) \oplus \mathfrak{s o}(1,1)$ & \multirow{3}{*}{$\mathrm{R}$} \\
\hline & $\mathfrak{s o}(3,7) \oplus \mathfrak{u}(1)$ & \\
\hline & $\mathfrak{s o}(9,1) \oplus \mathfrak{u}(1)$ & \\
\hline \multirow{4}{*}{$B_{2}+B_{3}$} & $\mathfrak{s o}(5) \oplus \mathfrak{s o}(4,3)$ & \multirow{4}{*}{$\mathrm{S}$} \\
\hline & $\mathfrak{s o}(2,3) \oplus \mathfrak{s o}(7)$ & \\
\hline & $\mathfrak{s o}(2,3) \oplus \mathfrak{s o}(6,1)$ & \\
\hline & $\mathfrak{s o}(4,1) \oplus \mathfrak{s o}(2,5)$ & \\
\hline \multirow{4}{*}{$A_{1}+B_{4}$} & $\mathfrak{s u}(2) \oplus \mathfrak{s o}(9)$ & \multirow{4}{*}{$\mathrm{S}$} \\
\hline & $\mathfrak{s u}(2) \oplus \mathfrak{s o}(6,3)$ & \\
\hline & $\mathfrak{s l}(2, \mathbb{R}) \oplus \mathfrak{s o}(2,7)$ & \\
\hline & $\mathfrak{s l}(2, \mathbb{R}) \oplus \mathfrak{s o}(8,1)$ & \\
\hline \multirow{2}{*}{$B_{5}$} & $\mathfrak{s o}(2,9)$ & \multirow{2}{*}{$\mathrm{S}$} \\
\hline & $\mathfrak{s o}(8,3)$ & \\
\hline
\end{tabular}

Table 73: Maximal subalgebras of $\mathfrak{s o}(5,7)$.

\begin{tabular}{|l|c|l|}
\hline type & real subalgebras & \\
\hline \multirow{3}{*}{$A_{3}+A_{3}$} & $\mathfrak{s u}(2,2) \oplus \mathfrak{s l}(4, \mathbb{R})$ & \\
& $\mathfrak{s u}(2,2) \oplus \mathfrak{s l}(2, \mathbb{H})$ & $\mathrm{R}$ \\
& $\mathfrak{s u}(4) \oplus \mathfrak{s l}(2, \mathbb{H})$ & \\
\hline
\end{tabular}




\begin{tabular}{|c|c|c|}
\hline \multicolumn{3}{|c|}{ subalgebras of $\mathfrak{s o}(5,7)$} \\
\hline \multirow{5}{*}{$A_{1}+A_{1}+D_{4}$} & $\mathfrak{s l}(2, \mathbb{R}) \oplus \mathfrak{s l}(2, \mathbb{R}) \oplus \mathfrak{s o}(3,5)$ & \multirow{5}{*}{$\mathrm{R}$} \\
\hline & $\mathfrak{s l}(2, \mathbb{C}) \oplus \mathfrak{s o}(4,4)$ & \\
\hline & $\mathfrak{s l}(2, \mathbb{C}) \oplus \mathfrak{s o}^{*}(8)$ & \\
\hline & $\mathfrak{s u}(2) \oplus \mathfrak{s u}(2) \oplus \mathfrak{s o}(3,5)$ & \\
\hline & $\mathfrak{s u}(2) \oplus \mathfrak{s u}(2) \oplus \mathfrak{s o}(1,7)$ & \\
\hline \multirow{3}{*}{$D_{5}+T_{1}$} & $\mathfrak{s o}(4,6) \oplus \mathfrak{s o}(1,1)$ & \multirow{3}{*}{$\mathrm{R}$} \\
\hline & $\mathfrak{s o}(5,5) \oplus \mathfrak{u}(1)$ & \\
\hline & $\mathfrak{s o}(3,7) \oplus \mathfrak{u}(1)$ & \\
\hline \multirow{6}{*}{$B_{2}+B_{3}$} & $\mathfrak{s o}(5) \oplus \mathfrak{s o}(7)$ & \multirow{6}{*}{$\mathrm{S}$} \\
\hline & $\mathfrak{s o}(5) \oplus \mathfrak{s o}(2,5)$ & \\
\hline & $\mathfrak{s o}(2,3) \oplus \mathfrak{s o}(2,5)$ & \\
\hline & $\mathfrak{s o}(2,3) \oplus \mathfrak{s o}(4,3)$ & \\
\hline & $\mathfrak{s o}(4,1) \oplus \mathfrak{s o}(4,3)$ & \\
\hline & $\mathfrak{s o}(4,1) \oplus \mathfrak{s o}(6,1)$ & \\
\hline \multirow{4}{*}{$A_{1}+B_{4}$} & $\mathfrak{s u}(2) \oplus \mathfrak{s o}(2,7)$ & \multirow{4}{*}{$\mathrm{S}$} \\
\hline & $\mathfrak{s u}(2) \oplus \mathfrak{s o}(4,5)$ & \\
\hline & $\mathfrak{s l}(2, \mathbb{R}) \oplus \mathfrak{s o}(4,5)$ & \\
\hline & $\mathfrak{s l}(2, \mathbb{R}) \oplus \mathfrak{s o}(6,3)$ & \\
\hline \multirow{2}{*}{$B_{5}$} & $\mathfrak{s o}(4,7)$ & \multirow{2}{*}{$\mathrm{S}$} \\
\hline & $\mathfrak{s o}(6,5)$ & \\
\hline
\end{tabular}

Type $E_{6}$

Table 74: Maximal subalgebras of $E_{6(6)}$.

\begin{tabular}{|l|c|l|}
\hline type & real subalgebras & \\
\hline$D_{5}+T_{1}$ & $\mathfrak{s o}(5,5) \oplus \mathfrak{s o}(1,1)$ & $\mathrm{R}$ \\
\hline$A_{2}+A_{2}+A_{2}$ & $\begin{array}{r}\mathfrak{s l}(3, \mathbb{R}) \oplus \mathfrak{s l}(3, \mathbb{R}) \oplus \mathfrak{s l}(3, \mathbb{R}) \\
\end{array}$ & $\mathrm{R}$ \\
\hline$A_{1}+A_{5}$ & $\mathfrak{s u}(1,2) \oplus \mathfrak{s l}(2, \mathbb{R}) \oplus \mathfrak{s l}(6, \mathbb{R})$ & \\
\hline$A_{2}+G_{2}$ & $\mathfrak{s u}(2) \oplus \mathfrak{s l}(3, \mathbb{H})$ & $\mathrm{R}$ \\
\hline \multirow{2}{*}{$C_{4}$} & $\mathfrak{s l}(3, \mathbb{R}) \oplus G_{2(2)}$ & $\mathrm{S}$ \\
\hline$F_{4}$ & $\mathfrak{s p}(4)$ & \\
& $\mathfrak{s p}(2,2)$ & $\mathrm{S}$ \\
& $F_{4(4)}(4, \mathbb{R})$ & \\
\hline
\end{tabular}

Table 75: Maximal subalgebras of $E_{6(2)}$.

\begin{tabular}{|l|r|l|}
\hline type & real subalgebras & \\
\hline$D_{5}+T_{1}$ & $\mathfrak{s o}(4,6) \oplus \mathfrak{u}(1)$ & $\mathrm{R}$ \\
& $\mathfrak{s o}(10) \oplus \mathfrak{u}(1)$ & \\
\hline & $\mathfrak{s l}(3, \mathbb{C}) \oplus \mathfrak{s l}(3, \mathbb{R})$ & \\
$A_{2}+A_{2}+A_{2}$ & $\mathfrak{s u}(1,2) \oplus \mathfrak{s u}(1,2) \oplus \mathfrak{s u}(1,2)$ & $\mathrm{R}$ \\
& $\mathfrak{s u}(3) \oplus \mathfrak{s u}(3) \oplus \mathfrak{s u}(1,2)$ & \\
\hline
\end{tabular}




\begin{tabular}{|c|c|c|}
\hline \multicolumn{3}{|c|}{ subalgebras of $E_{6(2)}$} \\
\hline$A_{1}+A_{5}$ & $\begin{array}{r}\mathfrak{s l}(2, \mathbb{R}) \oplus \mathfrak{s u}(3,3) \\
\mathfrak{s u}(2) \oplus \mathfrak{s u}(2,4) \\
\mathfrak{s u}(2) \oplus \mathfrak{s u}(6)\end{array}$ & $\mathrm{R}$ \\
\hline$A_{2}$ & $\begin{array}{l}\mathfrak{s u}(1,2) \\
\mathfrak{s l}(3, \mathbb{R})\end{array}$ & $\mathrm{S}$ \\
\hline$G_{2}$ & $G_{2(2)}$ & $\mathrm{S}$ \\
\hline$A_{2}+G_{2}$ & $\begin{array}{r}\mathfrak{s u}(3) \oplus G_{2(2)} \\
\mathfrak{s u}(1,2) \oplus G_{2(2)}\end{array}$ & $\mathrm{S}$ \\
\hline$C_{4}$ & $\begin{array}{l}\mathfrak{s p}(1,3) \\
\mathfrak{s p}(4, \mathbb{R})\end{array}$ & $\mathrm{S}$ \\
\hline$F_{4}$ & $F_{4(4)}$ & $\mathrm{S}$ \\
\hline
\end{tabular}

Table 76: Maximal subalgebras of $E_{6(-14)}$.

\begin{tabular}{|l|c|c|}
\hline type & real subalgebras & \\
\hline \multirow{3}{*}{$D_{5}+T_{1}$} & $\mathfrak{s o}^{*}(10) \oplus \mathfrak{u}(1)$ & \\
& $\mathfrak{s o}(2,8) \oplus \mathfrak{u}(1)$ & $\mathrm{R}$ \\
& $\mathfrak{s o}(10) \oplus \mathfrak{u}(1)$ & \\
\hline$A_{2}+A_{2}+A_{2}$ & $\mathfrak{s u}(3) \oplus \mathfrak{s u}(1,2) \oplus \mathfrak{s u}(1,2)$ & $\mathrm{R}$ \\
\hline$A_{1}+A_{5}$ & $\mathfrak{s l}(2, \mathbb{R}) \oplus \mathfrak{s u}(1,5)$ & $\mathrm{R}$ \\
& $\mathfrak{s u}(2) \oplus \mathfrak{s u}(2,4)$ & \\
\hline$A_{2}+G_{2}$ & $\mathfrak{s u}(1,2) \oplus G_{2}^{c}$ & $\mathrm{~S}$ \\
\hline$C_{4}$ & $\mathfrak{s p}(2,2)$ & $\mathrm{S}$ \\
\hline$F_{4}$ & $F_{4(-20)}$ & $\mathrm{S}$ \\
\hline
\end{tabular}

Table 77: Maximal subalgebras of $E_{6(-26)}$.

\begin{tabular}{|l|c|c|}
\hline type & real subalgebras & \\
\hline$D_{5}+T_{1}$ & $\mathfrak{s o}(9,1) \oplus \mathfrak{s o}(1,1)$ & $\mathrm{R}$ \\
\hline$A_{2}+A_{2}+A_{2}$ & $\mathfrak{s l}(3, \mathbb{C}) \oplus \mathfrak{s u}(3)$ & $\mathrm{R}$ \\
\hline$A_{1}+A_{5}$ & $\mathfrak{s u}(2) \oplus \mathfrak{s l}(3, \mathbb{H})$ & $\mathrm{R}$ \\
\hline$A_{2}+G_{2}$ & $\mathfrak{s l}(3, \mathbb{R}) \oplus G_{2}^{c}$ & $\mathrm{~S}$ \\
\hline$C_{4}$ & $\mathfrak{s p}(1,3)$ & $\mathrm{S}$ \\
\hline \multirow{2}{*}{$F_{4}$} & $F_{4}^{c}$ & $\mathrm{~S}$ \\
\hline
\end{tabular}

Type $A_{7}$

Table 78: Maximal subalgebras of $\mathfrak{s u}(1,7)$.

\begin{tabular}{|l|c|c|}
\hline type & real subalgebras & \\
\hline$A_{3}+A_{3}+T_{1}$ & $\mathfrak{s u}(4) \oplus \mathfrak{s u}(1,3) \oplus \mathfrak{u}(1)$ & $\mathrm{R}$ \\
\hline$A_{2}+A_{4}+T_{1}$ & $\begin{array}{c}\mathfrak{s u}(1,2) \oplus \mathfrak{s u}(5) \oplus \mathfrak{u}(1) \\
\mathfrak{s u}(3) \oplus \mathfrak{s u}(1,4) \oplus \mathfrak{u}(1)\end{array}$ & $\mathrm{R}$ \\
\hline$A_{1}+A_{5}+T_{1}$ & $\begin{array}{c}\mathfrak{s u}(2) \oplus \mathfrak{s u}(1,5) \oplus \mathfrak{u}(1) \\
\mathfrak{s l}(2, \mathbb{R}) \oplus \mathfrak{s u}(6) \oplus \mathfrak{u}(1)\end{array}$ & $\mathrm{R}$ \\
\hline$A_{6}+T_{1}$ & $\mathfrak{s u}(1,6) \oplus \mathfrak{u}(1)$ & $\mathrm{R}$ \\
& $\mathfrak{s u}(7) \oplus \mathfrak{u}(1)$ & \\
\hline
\end{tabular}




\begin{tabular}{|l|l|l|}
\hline \multicolumn{3}{|l|}{ subalgebras of $\mathfrak{s u}(1,7)$} \\
\hline$D_{4}$ & $\mathfrak{s o}(1,7)$ & $\mathrm{S}$ \\
\hline
\end{tabular}

Table 79: Maximal subalgebras of $\mathfrak{s u}(2,6)$.

\begin{tabular}{|l|r|l|}
\hline type & real subalgebras & \\
\hline$A_{3}+A_{3}+T_{1}$ & $\mathfrak{s u}(4) \oplus \mathfrak{s u}(2,2) \oplus \mathfrak{u}(1)$ & $\mathrm{R}$ \\
& $\mathfrak{s u}(1,3) \oplus \mathfrak{s u}(1,3) \oplus \mathfrak{u}(1)$ & \\
\hline & $\mathfrak{s u}(1,2) \oplus \mathfrak{s u}(1,4) \oplus \mathfrak{u}(1)$ & \\
$A_{2}+A_{4}+T_{1}$ & $\mathfrak{s u}(3) \oplus \mathfrak{s u}(2,3) \oplus \mathfrak{u}(1)$ & $\mathrm{R}$ \\
& $\mathfrak{s u}(1,2) \oplus \mathfrak{s u}(5) \oplus \mathfrak{u}(1)$ & \\
\hline & $\mathfrak{s u}(2) \oplus \mathfrak{s u}(2,4) \oplus \mathfrak{u}(1)$ & \\
$A_{1}+A_{5}+T_{1}$ & $\mathfrak{s l}(2, \mathbb{R}) \oplus \mathfrak{s u}(1,5) \oplus \mathfrak{u}(1)$ & $\mathrm{R}$ \\
& $\mathfrak{s u}(2) \oplus \mathfrak{s u}(6) \oplus \mathfrak{u}(1)$ & \\
\hline$A_{6}+T_{1}$ & $\mathfrak{s u}(2,5) \oplus \mathfrak{u}(1)$ & $\mathrm{R}$ \\
\hline$A_{1}+A_{3}$ & $\mathfrak{s u}(1,6) \oplus \mathfrak{u}(1)$ & \\
\hline$C_{4}$ & $\mathfrak{s u}(2) \oplus \mathfrak{s u}(1,3)$ & $\mathrm{S}$ \\
\hline
\end{tabular}

Table 80: Maximal subalgebras of $\mathfrak{s u}(3,5)$.

\begin{tabular}{|c|c|c|}
\hline type & real subalgebras & \\
\hline \multirow{2}{*}{$A_{3}+A_{3}+T_{1}$} & $\mathfrak{s} \mathfrak{u}(1,3) \oplus \mathfrak{s u}(2,2) \oplus \mathfrak{u}(1)$ & \multirow[b]{2}{*}{$\mathrm{R}$} \\
\hline & $\mathfrak{s u}(4) \oplus \mathfrak{s u}(1,3) \oplus \mathfrak{u}(1)$ & \\
\hline \multirow{4}{*}{$A_{2}+A_{4}+T_{1}$} & $\mathfrak{s} \mathfrak{u}(1,2) \oplus \mathfrak{s u}(2,3) \oplus \mathfrak{u}(1)$ & \multirow{4}{*}{$\mathrm{R}$} \\
\hline & $\mathfrak{s} \mathfrak{u}(1,2) \oplus \mathfrak{s} \mathfrak{u}(1,4) \oplus \mathfrak{u}(1)$ & \\
\hline & $\mathfrak{s u}(3) \oplus \mathfrak{s u}(2,3) \oplus \mathfrak{u}(1)$ & \\
\hline & $\mathfrak{s u}(3) \oplus \mathfrak{s u}(5) \oplus \mathfrak{u}(1)$ & \\
\hline \multirow{3}{*}{$A_{1}+A_{5}+T_{1}$} & $\mathfrak{s u}(2) \oplus \mathfrak{s u}(3,3) \oplus \mathfrak{u}(1)$ & \multirow{3}{*}{$\mathrm{R}$} \\
\hline & $\mathfrak{s l}(2, \mathbb{R}) \oplus \mathfrak{s} \mathfrak{u}(2,4) \oplus \mathfrak{u}(1)$ & \\
\hline & $\mathfrak{s u}(2) \oplus \mathfrak{s u}(1,5) \oplus \mathfrak{u}(1)$ & \\
\hline \multirow{2}{*}{$A_{6}+T_{1}$} & $\mathfrak{s u}(3,4) \oplus \mathfrak{u}(1)$ & \multirow{2}{*}{$\mathrm{R}$} \\
\hline & $\mathfrak{s u}(2,5) \oplus \mathfrak{u}(1)$ & \\
\hline$D_{4}$ & $\mathfrak{s o}(3,5)$ & $\mathrm{S}$ \\
\hline
\end{tabular}

Table 81: Maximal subalgebras of $\mathfrak{s u}(4,4)$.

\begin{tabular}{|c|c|c|}
\hline type & real subalgebras & \\
\hline \multirow{4}{*}{$A_{3}+A_{3}+T_{1}$} & $\mathfrak{s l}(4, \mathbb{C}) \oplus \mathfrak{s o}(1,1)$ & \\
\hline & $\mathfrak{s u}(2,2) \oplus \mathfrak{s u}(2,2) \oplus \mathfrak{u}(1)$ & \\
\hline & $\mathfrak{s u}(1,3) \oplus \mathfrak{s u}(1,3) \oplus \mathfrak{u}(1)$ & $\mathrm{R}$ \\
\hline & $\mathfrak{s u}(4) \oplus \mathfrak{s u}(4) \oplus \mathfrak{u}(1)$ & \\
\hline \multirow{2}{*}{$A_{2}+A_{4}+T_{1}$} & $\mathfrak{s u}(1,2) \oplus \mathfrak{s u}(2,3) \oplus \mathfrak{u}(1)(2 \times)$ & \\
\hline & $\mathfrak{s u}(3) \oplus \mathfrak{s} \mathfrak{u}(1,4) \oplus \mathfrak{u}(1)(2 \times)$ & R \\
\hline \multirow{2}{*}{$A_{1}+A_{5}+T_{1}$} & $\mathfrak{s l}(2, \mathbb{R}) \oplus \mathfrak{s u}(3,3) \oplus \mathfrak{u}(1)$ & \\
\hline & $\mathfrak{s u}(2) \oplus \mathfrak{s u}(2,4) \oplus \mathfrak{u}(1)(2 \times)$ & $\mathrm{n}$ \\
\hline$A_{6}+T_{1}$ & $\mathfrak{s u}(3,4) \oplus \mathfrak{u}(1)(2 \times)$ & $\mathrm{R}$ \\
\hline
\end{tabular}




\begin{tabular}{|c|c|c|}
\hline \multicolumn{3}{|c|}{ subalgebras of $\mathfrak{s u}(4,4)$} \\
\hline \multirow{4}{*}{$A_{1}+A_{3}$} & $\overline{\mathfrak{s u}}(2) \oplus \mathfrak{s u}(2,2)$ & \\
\hline & $\mathfrak{s l}(2, \mathbb{R}) \oplus \mathfrak{s u}(4)$ & \\
\hline & $\mathfrak{s l}(2, \mathbb{R}) \oplus \mathfrak{s u}(1,3)$ & $S$ \\
\hline & $\mathfrak{s l}(2, \mathbb{R}) \oplus \mathfrak{s u}(2,2)$ & \\
\hline \multirow[b]{2}{*}{$D_{4}$} & $\mathfrak{s o}^{*}(8)$ & \\
\hline & $\mathfrak{s o}(4,4)$ & S \\
\hline \multirow[b]{2}{*}{$C_{4}$} & $\mathfrak{s p}(2,2)$ & \\
\hline & $\mathfrak{s p}(4, \mathbb{R})$ & S \\
\hline
\end{tabular}

Table 82: Maximal subalgebras of $\mathfrak{s l}(4, \mathbb{H})$.

\begin{tabular}{|l|c|c|}
\hline type & real subalgebras & \\
\hline$A_{3}+A_{3}+T_{1}$ & $\mathfrak{s l}(4, \mathbb{C}) \oplus \mathfrak{u}(1)$ & $\mathrm{R}$ \\
& $\mathfrak{s l}(2, \mathbb{H}) \oplus \mathfrak{s l}(2, \mathbb{H}) \oplus \mathfrak{s o}(1,1)$ & \\
\hline$A_{1}+A_{5}+T_{1}$ & $\mathfrak{s u}(2) \oplus \mathfrak{s l}(3, \mathbb{H}) \oplus \mathfrak{s o}(1,1)$ & $\mathrm{R}$ \\
\hline$A_{1}+A_{3}+T_{1}$ & $\mathfrak{s u}(2) \oplus \mathfrak{s l}(4, \mathbb{R})$ & $\mathrm{S}$ \\
& $\mathfrak{s l}(2, \mathbb{R}) \oplus \mathfrak{s l}(2, \mathbb{H})$ & \\
\hline$D_{4}$ & $\mathfrak{s o}(8)$ & $\mathrm{S}$ \\
\hline & $\mathfrak{s p}(4)$ & $\mathrm{S}$ \\
$C_{4}$ & $\mathfrak{s p}(1,3)$ & \\
\hline
\end{tabular}

Table 83: Maximal subalgebras of $\mathfrak{s l}(8, \mathbb{R})$.

\begin{tabular}{|l|c|c|}
\hline type & real subalgebras & \\
\hline$A_{3}+A_{3}+T_{1}$ & $\mathfrak{s l}(4, \mathbb{R}) \oplus \mathfrak{s l}(4, \mathbb{R}) \oplus \mathfrak{s o}(1,1)$ & $\mathrm{R}$ \\
& $\mathfrak{s l}(4, \mathbb{C}) \oplus \mathfrak{u}(1)(2 \times)$ & \\
\hline$A_{2}+A_{4}+T_{1}$ & $\mathfrak{s l}(3, \mathbb{R}) \oplus \mathfrak{s l}(5, \mathbb{R}) \oplus \mathfrak{s o}(1,1)$ & $\mathrm{R}$ \\
\hline$A_{1}+A_{5}+T_{1}$ & $\mathfrak{s l}(2, \mathbb{R}) \oplus \mathfrak{s l}(6, \mathbb{R}) \oplus \mathfrak{s o}(1,1)$ & $\mathrm{R}$ \\
\hline$A_{6}+T_{1}$ & $\mathfrak{s l}(7, \mathbb{R}) \oplus \mathfrak{s o}(1,1)$ & $\mathrm{R}$ \\
\hline \multirow{2}{*}{$A_{1}+A_{3}$} & $\mathfrak{s u}(2) \oplus \mathfrak{s l}(2, \mathbb{H})$ & $\mathrm{S}$ \\
& $\mathfrak{s l}(2, \mathbb{R}) \oplus \mathfrak{s l}(4, \mathbb{R})$ & \\
& $\mathfrak{s o}(8)$ & \\
$D_{4}$ & $\mathfrak{s o}(4,4)$ & $\mathrm{S}$ \\
& $\mathfrak{s o}(3,5)$ & \\
\hline$C_{4}$ & $\mathfrak{s o}(1,7)$ & $\mathrm{S}$ \\
\hline
\end{tabular}

Type $B_{7}$

Table 84: Maximal subalgebras of $\mathfrak{s o}(2,13)$.

\begin{tabular}{|l|r|l|}
\hline type & real subalgebras & \\
\hline \multirow{3}{*}{$B_{3}+D_{4}$} & $\mathfrak{s o}(7) \oplus \mathfrak{s o}{ }^{*}(8)$ & \\
& $\mathfrak{s o}(6,1) \oplus \mathfrak{s o}(1,7)$ & $\mathrm{R}$ \\
& $\mathfrak{s o}(2,5) \oplus \mathfrak{s o}(8)$ & \\
\hline
\end{tabular}




\begin{tabular}{|l|r|l|}
\hline \multicolumn{2}{|c|}{ subalgebras of $\mathfrak{s o}(2,13)$} & \\
\hline \multirow{3}{*}{$A_{3}+B_{4}$} & $\mathfrak{s u}(2,2) \oplus \mathfrak{s o}(9)$ & \\
& $\mathfrak{s l}(2, \mathbb{H}) \oplus \mathfrak{s o}(8,1)$ & $\mathrm{R}$ \\
& $\mathfrak{s u}(4) \oplus \mathfrak{s o}(2,7)$ & \\
\hline \multirow{3}{*}{$B_{2}+D_{5}$} & $\mathfrak{s o}(5) \oplus \mathfrak{s o}(2,8)$ & \\
& $\mathfrak{s o}(4,1) \oplus \mathfrak{s o}(9,1)$ & $\mathrm{R}$ \\
\hline \multirow{3}{*}{$A_{1}+A_{1}+B_{5}$} & $\mathfrak{s o}(2,3) \oplus \mathfrak{s o}(10)$ & \\
& $\mathfrak{s l}(2, \mathbb{R}) \oplus \mathfrak{s l}(2, \mathbb{R}) \oplus \mathfrak{s o}(11)$ & \\
& $\mathfrak{s l}(2, \mathbb{C}) \oplus \mathfrak{s o}(10,1)$ & $\mathrm{R}$ \\
$A_{1}+D_{6}$ & $\mathfrak{s u}(2) \oplus \mathfrak{s u}(2) \oplus \mathfrak{s o}(2,9)$ & \\
\hline \multirow{2}{*}{$D_{7}$} & $\mathfrak{s l}(2, \mathbb{R}) \oplus \mathfrak{s o}(11,1)$ & $\mathrm{R}$ \\
& $\mathfrak{s l}(2, \mathbb{R}) \oplus \mathfrak{s o}(12)$ & \\
\hline \multirow{3}{*}{$B_{6}+T_{1}$} & $\mathfrak{s o}(2,12)$ & $\mathrm{R}$ \\
& $\mathfrak{s o}(13,1)$ & \\
& $\mathfrak{s o}(12,1) \oplus \mathfrak{s o}(1,1)$ & \\
& $\mathfrak{s o}(2,11) \oplus \mathfrak{u}(1)$ & $\mathrm{R}$ \\
& $\mathfrak{s o}(13) \oplus \mathfrak{u}(1)$ & \\
\hline
\end{tabular}

Table 85: Maximal subalgebras of $\mathfrak{s o}(4,11)$.

\begin{tabular}{|l|c|l|}
\hline type & real subalgebras & \\
\hline \multirow{5}{*}{$B_{3}+D_{4}$} & $\mathfrak{s o}(7) \oplus \mathfrak{s o}(4,4)$ & \\
& $\mathfrak{s o}(6,1) \oplus \mathfrak{s o}(3,5)$ & \\
& $\mathfrak{s o}(2,5) \oplus \mathfrak{s o}{ }^{*}(8)$ & $\mathrm{R}$ \\
& $\mathfrak{s o}(4,3) \oplus \mathfrak{s o}(1,7)$ & \\
& $\mathfrak{s o}(4,3) \oplus \mathfrak{s o}(8)$ & \\
\hline \multirow{5}{*}{$A_{3}+B_{4}$} & $\mathfrak{s l}(4, \mathbb{R}) \oplus \mathfrak{s o}(8,1)$ & \\
& $\mathfrak{s u}(2,2) \oplus \mathfrak{s o}(2,7)$ & \\
& $\mathfrak{s l}(2, \mathbb{H}) \oplus \mathfrak{s o}(6,3)$ & $\mathrm{R}$ \\
& $\mathfrak{s u}(4) \oplus \mathfrak{s o}(4,5)$ & \\
& $\mathfrak{s u}(2,2) \oplus \mathfrak{s o}(9)$ & \\
\hline \multirow{5}{*}{$B_{2}+D_{5}$} & $\mathfrak{s o}(5) \oplus \mathfrak{s o}(4,6)$ & \\
& $\mathfrak{s o}(4,1) \oplus \mathfrak{s o}(3,7)$ & \\
& $\mathfrak{s o}(2,3) \oplus \mathfrak{s o}(2,8)$ & $\mathrm{R}$ \\
& $\mathfrak{s o}(2,3) \oplus \mathfrak{s o}(9,1)$ & \\
& $\mathfrak{s o}(4,1) \oplus \mathfrak{s o}(10)$ & \\
\hline \multirow{3}{*}{$A_{1}+A_{1}+B_{5}$} & $\mathfrak{s l}(2, \mathbb{R}) \oplus \mathfrak{s l}(2, \mathbb{R}) \oplus \mathfrak{s o}(2,9)$ & \\
& $\mathfrak{s u}(2) \oplus \mathfrak{s u}(2) \oplus \mathfrak{s o}(4,7)$ & $\mathrm{R}$ \\
& $\mathfrak{s l}(2, \mathbb{C}) \oplus \mathfrak{s o}(10,1)$ & \\
& $\mathfrak{s u}(2) \oplus \mathfrak{s u}(2) \oplus \mathfrak{s o}(11)$ & \\
\hline
\end{tabular}




\begin{tabular}{|l|r|l|}
\hline \multicolumn{2}{|c|}{ subalgebras of $\mathfrak{s o}(4,11)$} \\
\hline \multirow{4}{*}{$A_{1}+D_{6}$} & $\mathfrak{s u}(2) \oplus \mathfrak{s o}(4,8)$ & \\
& $\mathfrak{s l}(2, \mathbb{R}) \oplus \mathfrak{s o}(3,9)$ & $\mathrm{R}$ \\
& $\mathfrak{s l}(2, \mathbb{R}) \oplus \mathfrak{s o}(2,10)$ & \\
\hline$D_{7}$ & $\mathfrak{s u}(2) \oplus \mathfrak{s o}(11,1)$ & \\
\hline \multirow{3}{*}{$B_{6}+T_{1}$} & $\mathfrak{s o}(4,10)$ & $\mathrm{R}$ \\
& $\mathfrak{s o}(3,11)$ & \\
& $\mathfrak{s o}(10,3) \oplus \mathfrak{s o}(1,1)$ & $\mathrm{R}$ \\
\hline
\end{tabular}

Table 86: Maximal subalgebras of $\mathfrak{s o}(6,9)$.

\begin{tabular}{|c|c|c|}
\hline type & real subalgebras & \\
\hline \multirow{7}{*}{$B_{3}+D_{4}$} & $\mathfrak{s o}(2,5) \oplus \mathfrak{s o}(4,4)$ & \multirow{7}{*}{$\mathrm{R}$} \\
\hline & $\mathfrak{s o}(4,3) \oplus \mathfrak{s o}(3,5)$ & \\
\hline & $\mathfrak{s o}(4,3) \oplus \mathfrak{s o}^{*}(8)$ & \\
\hline & $\mathfrak{s o}(6,1) \oplus \mathfrak{s o}(3,5)$ & \\
\hline & $\mathfrak{s o}(2,5) \oplus \mathfrak{s o}(1,7)$ & \\
\hline & $\mathfrak{s o}(7) \oplus \mathfrak{s o}^{*}(8)$ & \\
\hline & $\mathfrak{s o}(6,1) \oplus \mathfrak{s o}(8)$ & \\
\hline \multirow{7}{*}{$A_{3}+B_{4}$} & $\mathfrak{s l}(4, \mathbb{R}) \oplus \mathfrak{s o}(6,3)$ & \multirow{7}{*}{$\mathrm{R}$} \\
\hline & $\mathfrak{s u}(2,2) \oplus \mathfrak{s o}(4,5)$ & \\
\hline & $\mathfrak{s l}(2, \mathbb{H}) \oplus \mathfrak{s o}(4,5)$ & \\
\hline & $\mathfrak{s u}(2,2) \oplus \mathfrak{s o}(2,7)$ & \\
\hline & $\mathfrak{s u}(4) \oplus \mathfrak{s o}(6,3)$ & \\
\hline & $\mathfrak{s l}(2, \mathbb{H}) \oplus \mathfrak{s o}(8,1)$ & \\
\hline & $\mathfrak{s u}(4) \oplus \mathfrak{s o}(9)$ & \\
\hline \multirow{6}{*}{$B_{2}+D_{5}$} & $\mathfrak{s o}(4,1) \oplus \mathfrak{s o}(5,5)$ & \multirow{6}{*}{$\mathrm{R}$} \\
\hline & $\mathfrak{s o}(2,3) \oplus \mathfrak{s o}(4,6)$ & \\
\hline & $\mathfrak{s o}(2,3) \oplus \mathfrak{s o}(3,7)$ & \\
\hline & $\mathfrak{s o}(5) \oplus \mathfrak{s o}(4,6)$ & \\
\hline & $\mathfrak{s o}(4,1) \oplus \mathfrak{s o}(2,8)$ & \\
\hline & $\mathfrak{s o}(5) \oplus \mathfrak{s o}(9,1)$ & \\
\hline \multirow{5}{*}{$A_{1}+A_{1}+B_{5}$} & $\mathfrak{s l}(2, \mathbb{R}) \oplus \mathfrak{s l}(2, \mathbb{R}) \oplus \mathfrak{s o}(4,7)$ & \multirow{5}{*}{$\mathrm{R}$} \\
\hline & $\mathfrak{s l}(2, \mathbb{C}) \oplus \mathfrak{s o}(6,5)$ & \\
\hline & $\mathfrak{s u}(2) \oplus \mathfrak{s u}(2) \oplus \mathfrak{s o}(6,5)$ & \\
\hline & $\mathfrak{s l}(2, \mathbb{C}) \oplus \mathfrak{s o}(8,3)$ & \\
\hline & $\mathfrak{s u}(2) \oplus \mathfrak{s u}(2) \oplus \mathfrak{s o}(2,9)$ & \\
\hline \multirow{4}{*}{$A_{1}+D_{6}$} & $\mathfrak{s u}(2) \oplus \mathfrak{s o}(6,6)$ & \multirow{4}{*}{$\mathrm{R}$} \\
\hline & $\mathfrak{s l}(2, \mathbb{R}) \oplus \mathfrak{s o}(5,7)$ & \\
\hline & $\mathfrak{s l}(2, \mathbb{R}) \oplus \mathfrak{s o}(4,8)$ & \\
\hline & $\mathfrak{s u}(2) \oplus \mathfrak{s o}(3,9)$ & \\
\hline \multirow{2}{*}{$D_{7}$} & $\mathfrak{s o}(6,8)$ & \multirow{2}{*}{$\mathrm{R}$} \\
\hline & $\mathfrak{s o}(5,9)$ & \\
\hline
\end{tabular}




\begin{tabular}{|l|r|l|}
\hline \multicolumn{2}{|c|}{ subalgebras of $\mathfrak{s o}(6,9)$} \\
\hline \multirow{3}{*}{$B_{6}+T_{1}$} & $\mathfrak{s o}(8,5) \oplus \mathfrak{s o}(1,1)$ & \\
& $\mathfrak{s o}(6,7) \oplus \mathfrak{u}(1)$ & $\mathrm{R}$ \\
& $\mathfrak{s o}(4,9) \oplus \mathfrak{u}(1)$ & \\
\hline \multirow{2}{*}{$A_{1}+B_{2}$} & $\mathfrak{s u}(2) \oplus \mathfrak{s o}(2,3)$ & $\mathrm{S}$ \\
& $\mathfrak{s l}(2, \mathbb{R}) \oplus \mathfrak{s o}(4,1)$ & \\
\hline \multirow{2}{*}{$A_{3}$} & $\mathfrak{s u}(1,3)$ & $\mathrm{S}$ \\
& $\mathfrak{s l}(4, \mathbb{R})$ & \\
\hline
\end{tabular}

Table 87: Maximal subalgebras of $\mathfrak{s o}(8,7)$.

\begin{tabular}{|c|c|c|}
\hline type & real subalgebras & \\
\hline \multirow{8}{*}{$B_{3}+D_{4}$} & $\mathfrak{s o}(4,3) \oplus \mathfrak{s o}(4,4)$ & \multirow{8}{*}{$\mathrm{R}$} \\
\hline & $\mathfrak{s o}(4,3) \oplus \mathfrak{s o}(3,5)$ & \\
\hline & $\mathfrak{s o}(2,5) \oplus \mathfrak{s o}(3,5)$ & \\
\hline & $\mathfrak{s o}(2,5) \oplus \mathfrak{s o}^{*}(8)$ & \\
\hline & $\mathfrak{s o}(6,1) \oplus \mathfrak{s o}^{*}(8)$ & \\
\hline & $\mathfrak{s o}(6,1) \oplus \mathfrak{s o}(1,7)$ & \\
\hline & $\mathfrak{s o}(7) \oplus \mathfrak{s o}(1,7)$ & \\
\hline & $\mathfrak{s o}(7) \oplus \mathfrak{s o}(8)$ & \\
\hline \multirow{7}{*}{$A_{3}+B_{4}$} & $\mathfrak{s l}(4, \mathbb{R}) \oplus \mathfrak{s o}(4,5)$ & \multirow{7}{*}{$\mathrm{R}$} \\
\hline & $\mathfrak{s u}(2,2) \oplus \mathfrak{s o}(4,5)$ & \\
\hline & $\mathfrak{s u}(2,2) \oplus \mathfrak{s o}(6,3)$ & \\
\hline & $\mathfrak{s l}(2, \mathbb{H}) \oplus \mathfrak{s o}(6,3)$ & \\
\hline & $\mathfrak{s l}(2, \mathbb{H}) \oplus \mathfrak{s o}(2,7)$ & \\
\hline & $\mathfrak{s u}(4) \oplus \mathfrak{s o}(2,7)$ & \\
\hline & $\mathfrak{s u}(4) \oplus \mathfrak{s o}(8,1)$ & \\
\hline \multirow{6}{*}{$B_{2}+D_{5}$} & $\mathfrak{s o}(2,3) \oplus \mathfrak{s o}(5,5)$ & \multirow{6}{*}{$\mathrm{R}$} \\
\hline & $\mathfrak{s o}(2,3) \oplus \mathfrak{s o}(4,6)$ & \\
\hline & $\mathfrak{s o}(4,1) \oplus \mathfrak{s o}(4,6)$ & \\
\hline & $\mathfrak{s o}(4,1) \oplus \mathfrak{s o}(3,7)$ & \\
\hline & $\mathfrak{s o}(5) \oplus \mathfrak{s o}(3,7)$ & \\
\hline & $\mathfrak{s o}(5) \oplus \mathfrak{s o}(2,8)$ & \\
\hline \multirow{5}{*}{$A_{1}+A_{1}+B_{5}$} & $\mathfrak{s l}(2, \mathbb{R}) \oplus \mathfrak{s l}(2, \mathbb{R}) \oplus \mathfrak{s o}(6,5)$ & \multirow{5}{*}{$\mathrm{R}$} \\
\hline & $\mathfrak{s l}(2, \mathbb{C}) \oplus \mathfrak{s o}(6,5)$ & \\
\hline & $\mathfrak{s l}(2, \mathbb{C}) \oplus \mathfrak{s o}(4,7)$ & \\
\hline & $\mathfrak{s u}(2) \oplus \mathfrak{s u}(2) \oplus \mathfrak{s o}(4,7)$ & \\
\hline & $\mathfrak{s u}(2) \oplus \mathfrak{s u}(2) \oplus \mathfrak{s o}(8,3)$ & \\
\hline \multirow{4}{*}{$A_{1}+D_{6}$} & $\mathfrak{s l}(2, \mathbb{R}) \oplus \mathfrak{s o}(6,6)$ & \multirow{4}{*}{$\mathrm{R}$} \\
\hline & $\mathfrak{s l}(2, \mathbb{R}) \oplus \mathfrak{s o}(5,7)$ & \\
\hline & $\mathfrak{s u}(2) \oplus \mathfrak{s o}(5,7)$ & \\
\hline & $\mathfrak{s u}(2) \oplus \mathfrak{s o}(4,8)$ & \\
\hline \multirow{2}{*}{$D_{7}$} & $\mathfrak{s o}(7,7)$ & \multirow{2}{*}{$\mathrm{R}$} \\
\hline & $\mathfrak{s o}(6,8)$ & \\
\hline
\end{tabular}




\begin{tabular}{|l|r|l|}
\hline \multicolumn{2}{|c|}{ subalgebras of $\mathfrak{s o}(8,7)$} \\
\hline \multirow{3}{*}{$B_{6}+T_{1}$} & $\mathfrak{s o}(6,7) \oplus \mathfrak{s o}(1,1)$ & \\
& $\mathfrak{s o}(6,7) \oplus \mathfrak{u}(1)$ & $\mathrm{R}$ \\
& $\mathfrak{s o}(8,5) \oplus \mathfrak{u}(1)$ & \\
\hline$A_{1}$ & $\mathfrak{s l}(2, \mathbb{R})$ & $\mathrm{S}$ \\
\hline$A_{1}+B_{2}$ & $\mathfrak{s l}(2, \mathbb{R}) \oplus \mathfrak{s o}(2,3)$ & $\mathrm{S}$ \\
\hline$A_{3}$ & $\mathfrak{s u}(2,2)$ & $\mathrm{S}$ \\
\hline
\end{tabular}

Table 88: Maximal subalgebras of $\mathfrak{s o}(10,5)$.

\begin{tabular}{|c|c|c|}
\hline type & real subalgebras & \\
\hline \multirow{6}{*}{$B_{3}+D_{4}$} & $\mathfrak{s o}(6,1) \oplus \mathfrak{s o}(4,4)$ & \multirow{6}{*}{$\mathrm{R}$} \\
\hline & $\mathfrak{s o}(2,5) \oplus \mathfrak{s o}(3,5)$ & \\
\hline & $\mathfrak{s o}(4,3) \oplus \mathfrak{s o}^{*}(8)$ & \\
\hline & $\mathfrak{s o}(4,3) \oplus \mathfrak{s o}(1,7)$ & \\
\hline & $\mathfrak{s o}(7) \oplus \mathfrak{s o}(3,5)$ & \\
\hline & $\mathfrak{s o}(2,5) \oplus \mathfrak{s o}(8)$ & \\
\hline \multirow{6}{*}{$A_{3}+B_{4}$} & $\mathfrak{s l}(4, \mathbb{R}) \oplus \mathfrak{s o}(2,7)$ & \multirow{6}{*}{$\mathrm{R}$} \\
\hline & $\mathfrak{s u}(2,2) \oplus \mathfrak{s o}(6,3)$ & \\
\hline & $\mathfrak{s l}(2, \mathbb{H}) \oplus \mathfrak{s o}(4,5)$ & \\
\hline & $\mathfrak{s u}(4) \oplus \mathfrak{s o}(4,5)$ & \\
\hline & $\mathfrak{s u}(2,2) \oplus \mathfrak{s o}(8,1)$ & \\
\hline & $\mathfrak{s l}(2, \mathbb{H}) \oplus \mathfrak{s o}(9)$ & \\
\hline \multirow{6}{*}{$B_{2}+D_{5}$} & $\mathfrak{s o}(5) \oplus \mathfrak{s o}(5,5)$ & \multirow{6}{*}{$\mathrm{R}$} \\
\hline & $\mathfrak{s o}(4,1) \oplus \mathfrak{s o}(4,6)$ & \\
\hline & $\mathfrak{s o}(2,3) \oplus \mathfrak{s o}(3,7)$ & \\
\hline & $\mathfrak{s o}(2,3) \oplus \mathfrak{s o}(2,8)$ & \\
\hline & $\mathfrak{s o}(4,1) \oplus \mathfrak{s o}(9,1)$ & \\
\hline & $\mathfrak{s o}(5) \oplus \mathfrak{s o}(10)$ & \\
\hline \multirow{5}{*}{$A_{1}+A_{1}+B_{5}$} & $\mathfrak{s l}(2, \mathbb{R}) \oplus \mathfrak{s l}(2, \mathbb{R}) \oplus \mathfrak{s o}(8,3)$ & \multirow{5}{*}{$\mathrm{R}$} \\
\hline & $\mathfrak{s l}(2, \mathbb{C}) \oplus \mathfrak{s o}(4,7)$ & \\
\hline & $\mathfrak{s u}(2) \oplus \mathfrak{s u}(2) \oplus \mathfrak{s o}(6,5)$ & \\
\hline & $\mathfrak{s l}(2, \mathbb{C}) \oplus \mathfrak{s o}(2,9)$ & \\
\hline & $\mathfrak{s u}(2) \oplus \mathfrak{s u}(2) \oplus \mathfrak{s o}(10,1)$ & \\
\hline \multirow{4}{*}{$A_{1}+D_{6}$} & $\mathfrak{s u}(2) \oplus \mathfrak{s o}(5,7)$ & \multirow{4}{*}{$\mathrm{R}$} \\
\hline & $\mathfrak{s l}(2, \mathbb{R}) \oplus \mathfrak{s o}(4,8)$ & \\
\hline & $\mathfrak{s l}(2, \mathbb{R}) \oplus \mathfrak{s o}(3,9)$ & \\
\hline & $\mathfrak{s u}(2) \oplus \mathfrak{s o}(2,10)$ & \\
\hline \multirow{2}{*}{$D_{7}$} & $\mathfrak{s o}(5,9)$ & \multirow{2}{*}{$\mathrm{R}$} \\
\hline & $\mathfrak{s o}(4,10)$ & \\
\hline \multirow{3}{*}{$B_{6}+T_{1}$} & $\mathfrak{s o}(4,9) \oplus \mathfrak{s o}(1,1)$ & \multirow{3}{*}{$\mathrm{R}$} \\
\hline & $\mathfrak{s o}(8,5) \oplus \mathfrak{u}(1)$ & \\
\hline & $\mathfrak{s o}(10,3) \oplus \mathfrak{u}(1)$ & \\
\hline$A_{1}+B_{2}$ & $\mathfrak{s l}(2, \mathbb{R}) \oplus \mathfrak{s o}(5)$ & $\mathrm{S}$ \\
\hline$A_{3}$ & $\mathfrak{s l}(2, \mathbb{H})$ & $\mathrm{S}$ \\
\hline
\end{tabular}


Table 89: Maximal subalgebras of $\mathfrak{s o}(12,3)$.

\begin{tabular}{|c|c|c|}
\hline type & real subalgebras & \\
\hline \multirow{4}{*}{$B_{3}+D_{4}$} & $\mathfrak{s o}(7) \oplus \mathfrak{s o}(3,5)$ & \\
\hline & $\mathfrak{s o}(6,1) \oplus \mathfrak{s o}^{*}(8)$ & \\
\hline & $\mathfrak{s o}(2,5) \oplus \mathfrak{s o}(1,7)$ & $\mathrm{R}$ \\
\hline & $\mathfrak{s o}(4,3) \oplus \mathfrak{s o}(8)$ & \\
\hline \multirow{4}{*}{$A_{3}+B_{4}$} & $\mathfrak{s l}(4, \mathbb{R}) \oplus \mathfrak{s o}(9)$ & \\
\hline & $\mathfrak{s u}(2,2) \oplus \mathfrak{s o}(8,1)$ & \\
\hline & $\mathfrak{s u}(4) \oplus \mathfrak{s o}(6,3)$ & $\mathrm{R}$ \\
\hline & $\mathfrak{s l}(2, \mathbb{H}) \oplus \mathfrak{s o}(2,7)$ & \\
\hline \multirow{4}{*}{$B_{2}+D_{5}$} & $\mathfrak{s o}(5) \oplus \mathfrak{s o}(3,7)$ & \\
\hline & $\mathfrak{s o}(4,1) \oplus \mathfrak{s o}(2,8)$ & \\
\hline & $\mathfrak{s o}(2,3) \oplus \mathfrak{s o}(9,1)$ & $\mathrm{R}$ \\
\hline & $\mathfrak{s o}(2,3) \oplus \mathfrak{s o}(10)$ & \\
\hline \multirow{4}{*}{$A_{1}+A_{1}+B_{5}$} & $\mathfrak{s l}(2, \mathbb{R}) \oplus \mathfrak{s l}(2, \mathbb{R}) \oplus \mathfrak{s o}(10,1)$ & \\
\hline & $\mathfrak{s l}(2, \mathbb{C}) \oplus \mathfrak{s o}(2,9)$ & \\
\hline & $\mathfrak{s u}(2) \oplus \mathfrak{s u}(2) \oplus \mathfrak{s o}(8,3)$ & $\mathrm{K}$ \\
\hline & $\mathfrak{s l}(2, \mathbb{C}) \oplus \mathfrak{s o}(11)$ & \\
\hline \multirow{4}{*}{$A_{1}+D_{6}$} & $\mathfrak{s u}(2) \oplus \mathfrak{s o}(3,9)$ & \\
\hline & $\mathfrak{s l}(2, \mathbb{R}) \oplus \mathfrak{s o}(2,10)$ & \\
\hline & $\mathfrak{s l}(2, \mathbb{R}) \oplus \mathfrak{s o}(11,1)$ & $\mathrm{R}$ \\
\hline & $\mathfrak{s u}(2) \oplus \mathfrak{s o}(12)$ & \\
\hline \multirow{2}{*}{$D_{7}$} & $\mathfrak{s o}(3,11)$ & \\
\hline & & $\mathrm{R}$ \\
\hline \multirow{3}{*}{$B_{6}+T_{1}$} & $\mathfrak{s o}(2,11) \oplus \mathfrak{s o}(1,1)$ & \\
\hline & $\mathfrak{s o}(10,3) \oplus \mathfrak{u}(1)$ & $\mathrm{R}$ \\
\hline & $\mathfrak{s o}(12,1) \oplus \mathfrak{u}(1)$ & \\
\hline$A_{1}+B_{2}$ & $\mathfrak{s u}(2) \oplus \mathfrak{s o}(4,1)$ & $\mathrm{S}$ \\
\hline
\end{tabular}

Table 90: Maximal subalgebras of $\mathfrak{s o}(14,1)$.

\begin{tabular}{|l|c|l|}
\hline type & real subalgebras & \\
\hline$B_{3}+D_{4}$ & $\begin{array}{c}\mathfrak{s o}(7) \oplus \mathfrak{s o}(1,7) \\
\mathfrak{s o}(6,1) \oplus \mathfrak{s o}(8)\end{array}$ & $\mathrm{R}$ \\
\hline$A_{3}+B_{4}$ & $\begin{array}{c}\mathfrak{s l}(2, \mathbb{H}) \oplus \mathfrak{s o}(9) \\
\mathfrak{s u}(4) \oplus \mathfrak{s o}(8,1)\end{array}$ & $\mathrm{R}$ \\
\hline$B_{2}+D_{5}$ & $\begin{array}{r}\mathfrak{s o}(5) \oplus \mathfrak{s o}(9,1) \\
\mathfrak{s o}(4,1) \oplus \mathfrak{s o}(10)\end{array}$ & $\mathrm{R}$ \\
\hline$A_{1}+A_{1}+B_{5}$ & $\mathfrak{s l}(2, \mathbb{C}) \oplus \mathfrak{s o}(11)$ & $\mathrm{R}$ \\
\hline$A_{1}+D_{6}$ & $\begin{array}{c}\mathfrak{s u}(2) \oplus \mathfrak{s u}(2) \oplus \mathfrak{s o}(10,1) \\
\mathfrak{s}(2, \mathbb{R}) \oplus \mathfrak{s}(11,1)\end{array}$ & $\mathrm{R}$ \\
\hline$D_{7}$ & $\mathfrak{s o}(13,1)$ & $\mathrm{R}$ \\
\hline
\end{tabular}




\begin{tabular}{|l|r|l|}
\hline \multicolumn{3}{|c|}{ subalgebras of $\mathfrak{s o}(14,1)$} \\
\hline$B_{6}+T_{1}$ & $\mathfrak{s o}(13) \oplus \mathfrak{s o}(1,1)$ & $\mathrm{R}$ \\
& $\mathfrak{s o}(12,1) \oplus \mathfrak{u}(1)$ & \\
\hline
\end{tabular}

\section{Type $C_{7}$}

Table 91: Maximal subalgebras of $\mathfrak{s p}(1,6)$.

\begin{tabular}{|l|c|c|}
\hline type & real subalgebras & \\
\hline$A_{6}+T_{1}$ & $\mathfrak{s u}(1,6) \oplus \mathfrak{u}(1)$ & $\mathrm{R}$ \\
\hline$C_{3}+C_{4}$ & $\mathfrak{s p}(1,2) \oplus \mathfrak{s p}(4)$ & $\mathrm{R}$ \\
& $\mathfrak{s p}(3) \oplus \mathfrak{s p}(1,3)$ & \\
\hline$B_{2}+C_{5}$ & $\mathfrak{s o}(4,1) \oplus \mathfrak{s p}(5)$ & $\mathrm{R}$ \\
\hline$A_{1}+C_{6}$ & $\mathfrak{s u}(2) \oplus \mathfrak{s p}(1,5)$ & $\mathrm{R}$ \\
& $\mathfrak{s u}(2) \oplus \mathfrak{s p}(6)$ & \\
\hline$A_{1}+B_{3}$ & $\mathfrak{s u}(2) \oplus \mathfrak{s o}(6,1)$ & $\mathrm{S}$ \\
\hline
\end{tabular}

Table 92: Maximal subalgebras of $\mathfrak{s p}(2,5)$.

\begin{tabular}{|c|c|c|}
\hline type & real subalgebras & \\
\hline$A_{6}+T_{1}$ & $\mathfrak{s u}(2,5) \oplus \mathfrak{u}(1)$ & $\mathrm{R}$ \\
\hline \multirow{3}{*}{$C_{3}+C_{4}$} & $\mathfrak{s p}(1,2) \oplus \mathfrak{s p}(1,3)$ & \multirow{3}{*}{$\mathrm{R}$} \\
\hline & $\mathfrak{s p}(3) \oplus \mathfrak{s p}(2,2)$ & \\
\hline & $\mathfrak{s p}(1,2) \oplus \mathfrak{s p}(4)$ & \\
\hline \multirow{3}{*}{$B_{2}+C_{5}$} & $\mathfrak{s o}(4,1) \oplus \mathfrak{s p}(1,4)$ & \multirow{3}{*}{$\mathrm{R}$} \\
\hline & $\mathfrak{s o}(5) \oplus \mathfrak{s p}(2,3)$ & \\
\hline & $\mathfrak{s o}(5) \oplus \mathfrak{s p}(5)$ & \\
\hline \multirow{2}{*}{$A_{1}+C_{6}$} & $\mathfrak{s u}(2) \oplus \mathfrak{s p}(2,4)$ & \multirow{2}{*}{$\mathrm{R}$} \\
\hline & $\mathfrak{s u}(2) \oplus \mathfrak{s p}(1,5)$ & \\
\hline$C_{3}$ & $\mathfrak{s p}(1,2)$ & $\mathrm{S}$ \\
\hline$A_{1}+B_{3}$ & $\mathfrak{s u}(2) \oplus \mathfrak{s o}(2,5)$ & $\mathrm{S}$ \\
\hline
\end{tabular}

Table 93: Maximal subalgebras of $\mathfrak{s p}(3,4)$.

\begin{tabular}{|l|r|l|}
\hline type & real subalgebras & \\
\hline$A_{6}+T_{1}$ & $\mathfrak{s u}(3,4) \oplus \mathfrak{u}(1)$ & $\mathrm{R}$ \\
\hline & $\mathfrak{s p}(1,2) \oplus \mathfrak{s p}(2,2)$ & \\
& $\mathfrak{s p}(1,2) \oplus \mathfrak{s p}(1,3)$ & \\
$C_{3}+C_{4}$ & $\mathfrak{s p}(3) \oplus \mathfrak{s p}(1,3)$ & $\mathrm{R}$ \\
& $\mathfrak{s p}(3) \oplus \mathfrak{s p}(4)$ & \\
\hline & $\mathfrak{s o}(4,1) \oplus \mathfrak{s p}(2,3)$ & \\
$B_{2}+C_{5}$ & $\mathfrak{s o}(5) \oplus \mathfrak{s p}(2,3)$ & $\mathrm{R}$ \\
& $\mathfrak{s o}(5) \oplus \mathfrak{s p}(1,4)$ & \\
\hline \multirow{2}{*}{$A_{1}+C_{6}$} & $\mathfrak{s u}(2) \oplus \mathfrak{s p}(3,3)$ & $\mathrm{R}$ \\
& $\mathfrak{s u}(2) \oplus \mathfrak{s p}(2,4)$ & \\
\hline$A_{1}+B_{3}$ & $\mathfrak{s u}(2) \oplus \mathfrak{s o}(4,3)$ & $\mathrm{S}$ \\
\hline
\end{tabular}


Table 94: Maximal subalgebras of $\mathfrak{s p}(7, \mathbb{R})$.

\begin{tabular}{|l|r|l|}
\hline type & real subalgebras & \\
\hline & $\mathfrak{s l}(7, \mathbb{R}) \oplus \mathfrak{s o}(1,1)$ & \\
& $\mathfrak{s u}(3,4) \oplus \mathfrak{u}(1)$ & \\
$A_{6}+T_{1}$ & $\mathfrak{s u}(2,5) \oplus \mathfrak{u}(1)$ & $\mathrm{R}$ \\
& $\mathfrak{s u}(1,6) \oplus \mathfrak{u}(1)$ & \\
& $\mathfrak{s u}(7) \oplus \mathfrak{u}(1)$ & \\
\hline$C_{3}+C_{4}$ & $\mathfrak{s p}(3, \mathbb{R}) \oplus \mathfrak{s p}(4, \mathbb{R})$ & $\mathrm{R}$ \\
\hline$B_{2}+C_{5}$ & $\mathfrak{s o}(2,3) \oplus \mathfrak{s p}(5, \mathbb{R})$ & $\mathrm{R}$ \\
\hline$A_{1}+C_{6}$ & $\mathfrak{s l}(2, \mathbb{R}) \oplus \mathfrak{s p}(6, \mathbb{R})$ & $\mathrm{R}$ \\
\hline$A_{1}$ & $\mathfrak{s l}(2, \mathbb{R})$ & $\mathrm{S}$ \\
\hline$C_{3}$ & $\mathfrak{s p}(3, \mathbb{R})$ & $\mathrm{S}$ \\
\hline & $\mathfrak{s l}(2, \mathbb{R}) \oplus \mathfrak{s o}(7)$ & \\
& $\mathfrak{s l}(2, \mathbb{R}) \oplus \mathfrak{s o}(2,5)$ & $\mathrm{S}$ \\
$A_{1}+B_{3}$ & $\mathfrak{s l}(2, \mathbb{R}) \oplus \mathfrak{s o}(4,3)$ & \\
& $\mathfrak{s l}(2, \mathbb{R}) \oplus \mathfrak{s o}(6,1)$ & \\
\hline \multicolumn{3}{|c}{}
\end{tabular}

Type $D_{7}$

Table 95: Maximal subalgebras of $\mathfrak{s o}(2,12)$.

\begin{tabular}{|c|c|c|}
\hline type & real subalgebras & \\
\hline$A_{6}+T_{1}$ & $\mathfrak{s} \mathfrak{u}(1,6) \oplus \mathfrak{u}(1)(2 \times)$ & $\mathrm{R}$ \\
\hline \multirow{3}{*}{$A_{3}+D_{4}$} & $\mathfrak{s u}(2,2) \oplus \mathfrak{s o}(8)$ & \\
\hline & $\mathfrak{s l}(2, \mathbb{H}) \oplus \mathfrak{s o}(1,7)$ & $\mathrm{R}$ \\
\hline & $\mathfrak{s u}(4) \oplus \mathfrak{s o}^{*}(8)$ & \\
\hline \multirow{3}{*}{$A_{1}+A_{1}+D_{5}$} & $\mathfrak{s l}(2, \mathbb{R}) \oplus \mathfrak{s l}(2, \mathbb{R}) \oplus \mathfrak{s o}(10)$ & \\
\hline & $\mathfrak{s l}(2, \mathbb{C}) \oplus \mathfrak{s o}(9,1)$ & $\mathrm{R}$ \\
\hline & $\mathfrak{s u}(2) \oplus \mathfrak{s u}(2) \oplus \mathfrak{s o}(2,8)$ & \\
\hline \multirow{3}{*}{$D_{6}+T_{1}$} & $\mathfrak{s o}(11,1) \oplus \mathfrak{s o}(1,1)$ & \\
\hline & $\mathfrak{s o}(2,10) \oplus \mathfrak{u}(1)$ & $\mathrm{R}$ \\
\hline & $\mathfrak{s o}(12) \oplus \mathfrak{u}(1)$ & \\
\hline \multirow{2}{*}{$B_{3}+B_{3}$} & $\mathfrak{s o}(7) \oplus \mathfrak{s o}(2,5)$ & \\
\hline & $\mathfrak{s o}(6,1) \oplus \mathfrak{s o}(6,1)$ & $S$ \\
\hline \multirow{3}{*}{$B_{2}+B_{4}$} & $\mathfrak{s o}(5) \oplus \mathfrak{s o}(2,7)$ & \\
\hline & $\mathfrak{s o}(2,3) \oplus \mathfrak{s o}(9)$ & $\mathrm{S}$ \\
\hline & $\mathfrak{s o}(4,1) \oplus \mathfrak{s o}(8,1)$ & \\
\hline \multirow{3}{*}{$A_{1}+B_{5}$} & $\mathfrak{s u}(2) \oplus \mathfrak{s o}(2,9)$ & \\
\hline & $\mathfrak{s l}(2, \mathbb{R}) \oplus \mathfrak{s o}(11)$ & $\mathrm{S}$ \\
\hline & $\mathfrak{s l}(2, \mathbb{R}) \oplus \mathfrak{s o}(10,1)$ & \\
\hline \multirow{2}{*}{$B_{6}$} & $\mathfrak{s o}(2,11)$ & $\mathrm{S}$ \\
\hline & $\mathfrak{s o}(12,1)$ & $\mathrm{N}$ \\
\hline
\end{tabular}

Table 96: Maximal subalgebras of $\mathfrak{s o}(4,10)$.

\begin{tabular}{|l|c|c|}
\hline type & real subalgebras & \\
\hline$A_{6}+T_{1}$ & $\mathfrak{s u}(2,5) \oplus \mathfrak{u}(1)(2 \times)$ & $\mathrm{R}$ \\
\hline
\end{tabular}




\begin{tabular}{|c|c|c|}
\hline \multicolumn{3}{|c|}{ subalgebras of $\mathfrak{s o}(4,10)$} \\
\hline \multirow{5}{*}{$A_{3}+D_{4}$} & $\mathfrak{s l}(4, \mathbb{R}) \oplus \mathfrak{s o}(1,7)$ & \\
\hline & $\mathfrak{s u}(2,2) \oplus \mathfrak{s o}^{*}(8)$ & \\
\hline & $\mathfrak{s l}(2, \mathbb{H}) \oplus \mathfrak{s o}(3,5)$ & $\mathrm{R}$ \\
\hline & $\mathfrak{s u}(4) \oplus \mathfrak{s o}(4,4)$ & \\
\hline & $\mathfrak{s u}(2,2) \oplus \mathfrak{s o}(8)$ & \\
\hline \multirow{5}{*}{$A_{1}+A_{1}+D_{5}$} & $\mathfrak{s l}(2, \mathbb{R}) \oplus \mathfrak{s l}(2, \mathbb{R}) \oplus \mathfrak{s o}(2,8)$ & \\
\hline & $\mathfrak{s l}(2, \mathbb{C}) \oplus \mathfrak{s o}(3,7)$ & \\
\hline & $\mathfrak{s u}(2) \oplus \mathfrak{s u}(2) \oplus \mathfrak{s o}(4,6)$ & $\mathrm{R}$ \\
\hline & $\mathfrak{s l}(2, \mathbb{C}) \oplus \mathfrak{s o}(9,1)$ & \\
\hline & $\mathfrak{s u}(2) \oplus \mathfrak{s u}(2) \oplus \mathfrak{s o}(10)$ & \\
\hline \multirow{3}{*}{$D_{6}+T_{1}$} & $\mathfrak{s o}(3,9) \oplus \mathfrak{s o}(1,1)$ & \\
\hline & $\mathfrak{s o}(4,8) \oplus \mathfrak{u}(1)$ & $\mathrm{R}$ \\
\hline & $\mathfrak{s o}(2,10) \oplus \mathfrak{u}(1)$ & \\
\hline$B_{2}$ & $\mathfrak{s o}(4,1)$ & $\mathrm{S}$ \\
\hline \multirow{2}{*}{$B_{3}+B_{3}$} & $\mathfrak{s o}(7) \oplus \mathfrak{s o}(4,3)$ & $\mathrm{S}$ \\
\hline & $\mathfrak{s o}(2,5) \oplus \mathfrak{s o}(2,5)$ & $S$ \\
\hline \multirow{5}{*}{$B_{2}+B_{4}$} & $\mathfrak{s o}(5) \oplus \mathfrak{s o}(4,5)$ & \\
\hline & $\mathfrak{s o}(2,3) \oplus \mathfrak{s o}(2,7)$ & \\
\hline & $\mathfrak{s o}(2,3) \oplus \mathfrak{s o}(8,1)$ & $\mathrm{S}$ \\
\hline & $\mathfrak{s o}(4,1) \oplus \mathfrak{s o}(9)$ & \\
\hline & $\mathfrak{s o}(4,1) \oplus \mathfrak{s o}(6,3)$ & \\
\hline \multirow{4}{*}{$A_{1}+B_{5}$} & $\mathfrak{s u}(2) \oplus \mathfrak{s o}(4,7)$ & \\
\hline & $\mathfrak{s u}(2) \oplus \mathfrak{s o}(10,1)$ & \\
\hline & $\mathfrak{s l}(2, \mathbb{R}) \oplus \mathfrak{s o}(2,9)$ & $S$ \\
\hline & $\mathfrak{s l}(2, \mathbb{R}) \oplus \mathfrak{s o}(8,3)$ & \\
\hline \multirow{2}{*}{$B_{6}$} & $\mathfrak{s o}(4,9)$ & \\
\hline & $\mathfrak{s o}(10,3)$ & $S$ \\
\hline
\end{tabular}

Table 97: Maximal subalgebras of $\mathfrak{s o}(6,8)$.

\begin{tabular}{|l|r|l|}
\hline type & real subalgebras & \\
\hline$A_{6}+T_{1}$ & $\mathfrak{s u}(3,4) \oplus \mathfrak{u}(1)(2 \times)$ & $\mathrm{R}$ \\
\hline & $\mathfrak{s l}(4, \mathbb{R}) \oplus \mathfrak{s o}(3,5)$ & \\
& $\mathfrak{s u}(2,2) \oplus \mathfrak{s o}(4,4)$ & \\
& $\mathfrak{s u}(2,2) \oplus \mathfrak{s o} *^{*}(8)$ & \\
$A_{3}+D_{4}$ & $\mathfrak{s l}(2, \mathbb{H}) \oplus \mathfrak{s o}(3,5)$ & $\mathrm{R}$ \\
& $\mathfrak{s l}(2, \mathbb{H}) \oplus \mathfrak{s o}(1,7)$ & \\
& $\mathfrak{s u}(4) \oplus \mathfrak{s o} *^{*}(8)$ & \\
& $\mathfrak{s u}(4) \oplus \mathfrak{s o}(8)$ & \\
\hline \multirow{3}{*}{$A_{1}+A_{1}+D_{5}$} & $\mathfrak{s l}(2, \mathbb{R}) \oplus \mathfrak{s l}(2, \mathbb{R}) \oplus \mathfrak{s o}(4,6)$ & \\
& $\mathfrak{s l}(2, \mathbb{C}) \oplus \mathfrak{s o}(5,5)$ & \\
& $\mathfrak{s u}(2) \oplus \mathfrak{s u}(2) \oplus \mathfrak{s o}(4,6)$ & $\mathrm{R}$ \\
& $\mathfrak{s u}(2) \oplus \mathfrak{s u}(2) \oplus \mathfrak{s o}(2,8)$ & \\
\hline
\end{tabular}




\begin{tabular}{|c|c|c|}
\hline \multicolumn{3}{|c|}{ subalgebras of $\mathfrak{s o}(6,8)$} \\
\hline \multirow{3}{*}{$D_{6}+T_{1}$} & $\mathfrak{s o}(5,7) \oplus \mathfrak{s o}(1,1)$ & \multirow{3}{*}{$\mathrm{R}$} \\
\hline & $\mathfrak{s o}(6,6) \oplus \mathfrak{u}(1)$ & \\
\hline & $\mathfrak{s o}(4,8) \oplus \mathfrak{u}(1)$ & \\
\hline$B_{2}$ & $\mathfrak{s o}(2,3)$ & $\mathrm{S}$ \\
\hline$G_{2}$ & $G_{2(2)}$ & $\mathrm{S}$ \\
\hline \multirow{2}{*}{$C_{3}$} & $\mathfrak{s p}(1,2)$ & \multirow{2}{*}{$\mathrm{S}$} \\
\hline & $\mathfrak{s p}(3, \mathbb{R})$ & \\
\hline \multirow{4}{*}{$B_{3}+B_{3}$} & $\mathfrak{s o}(7) \oplus \mathfrak{s o}(6,1)$ & \multirow{4}{*}{$\mathrm{S}$} \\
\hline & $\mathfrak{s o}(2,5) \oplus \mathfrak{s o}(4,3)$ & \\
\hline & $\mathfrak{s o}(2,5) \oplus \mathfrak{s o}(6,1)$ & \\
\hline & $\mathfrak{s o}(4,3) \oplus \mathfrak{s o}(4,3)$ & \\
\hline \multirow{5}{*}{$B_{2}+B_{4}$} & $\mathfrak{s o}(5) \oplus \mathfrak{s o}(6,3)$ & \multirow{5}{*}{$\mathrm{S}$} \\
\hline & $\mathfrak{s o}(5) \oplus \mathfrak{s o}(8,1)$ & \\
\hline & $\mathfrak{s o}(2,3) \oplus \mathfrak{s o}(4,5)$ & \\
\hline & $\mathfrak{s o}(2,3) \oplus \mathfrak{s o}(6,3)$ & \\
\hline & $\mathfrak{s o}(4,1) \oplus \mathfrak{s o}(2,7)$ & \\
\hline \multirow{4}{*}{$A_{1}+B_{5}$} & $\mathfrak{s u}(2) \oplus \mathfrak{s o}(6,5)$ & \multirow{4}{*}{$\mathrm{S}$} \\
\hline & $\mathfrak{s u}(2) \oplus \mathfrak{s o}(8,3)$ & \\
\hline & $\mathfrak{s l}(2, \mathbb{R}) \oplus \mathfrak{s o}(4,7)$ & \\
\hline & $\mathfrak{s l}(2, \mathbb{R}) \oplus \mathfrak{s o}(6,5)$ & \\
\hline \multirow{2}{*}{$B_{6}$} & $\mathfrak{s o}(6,7)$ & \multirow{2}{*}{$\mathrm{S}$} \\
\hline & $\mathfrak{s o}(8,5)$ & \\
\hline
\end{tabular}

Table 98: Maximal subalgebras of $\mathfrak{s o}^{*}(14)$.

\begin{tabular}{|l|c|l|}
\hline type & real subalgebras & \\
\hline & $\mathfrak{s u}(3,4) \oplus \mathfrak{u}(1)$ & \\
$A_{6}+T_{1}$ & $\mathfrak{s u}(2,5) \oplus \mathfrak{u}(1)$ & \\
& $\mathfrak{s u}(1,6) \oplus \mathfrak{u}(1)$ & $\mathrm{R}$ \\
& $\mathfrak{s u}(7) \oplus \mathfrak{u}(1)$ & \\
\hline$A_{3}+D_{4}$ & $\mathfrak{s u}(1,3) \oplus \mathfrak{s o}^{*}(8)$ & $\mathrm{R}$ \\
\hline$A_{1}+A_{1}+D_{5}$ & $\mathfrak{s u}(2) \oplus \mathfrak{s l}(2, \mathbb{R}) \oplus \mathfrak{s o}^{*}(10)$ & $\mathrm{R}$ \\
\hline$D_{6}+T_{1}$ & $\mathfrak{s o}(12) \oplus \mathfrak{u}(1)$ & $\mathrm{R}$ \\
\hline
\end{tabular}

Table 99: Maximal subalgebras of $\mathfrak{s o}(13,1)$.

\begin{tabular}{|c|c|c|}
\hline type & real subalgebras & \\
\hline \multirow{2}{*}{$A_{3}+D_{4}$} & $\mathfrak{s u}(4) \oplus \mathfrak{s o}(1,7)$ & \\
\hline & $\mathfrak{s l}(2, \mathbb{H}) \oplus \mathfrak{s o}(8)$ & $\mathrm{K}$ \\
\hline \multirow[b]{2}{*}{$A_{1}+A_{1}+D_{5}$} & $\mathfrak{s u}(2) \oplus \mathfrak{s u}(2) \oplus \mathfrak{s o}(9,1)$ & \\
\hline & $\mathfrak{s l}(2, \mathbb{C}) \oplus \mathfrak{s o}(10)$ & $\mathrm{R}$ \\
\hline \multirow{2}{*}{$D_{6}+T_{1}$} & $\mathfrak{s o}(11,1) \oplus \mathfrak{u}(1)$ & \\
\hline & $\mathfrak{s o}(12) \oplus \mathfrak{s o}(1,1)$ & $\mathrm{R}$ \\
\hline$B_{3}+B_{3}$ & $\mathfrak{s o}(7) \oplus \mathfrak{s o}(6,1)$ & $\mathrm{S}$ \\
\hline \multirow{2}{*}{$B_{2}+B_{4}$} & $\mathfrak{s o}(5) \oplus \mathfrak{s o}(8,1)$ & $\mathrm{S}$ \\
\hline & $\mathfrak{s o}(4,1) \oplus \mathfrak{s o}(9)$ & $S$ \\
\hline
\end{tabular}




\begin{tabular}{|l|c|c|}
\hline \multicolumn{2}{|l|}{ subalgebras of $\mathfrak{s o}(13,1)$} \\
\hline$A_{1}+B_{5}$ & $\begin{array}{c}\mathfrak{s u}(2) \oplus \mathfrak{s o}(10,1) \\
\mathfrak{s l}(2, \mathbb{R}) \oplus \mathfrak{s o}(11)\end{array}$ & $\mathrm{S}$ \\
\hline$B_{6}$ & $\begin{array}{c}\mathfrak{s o}(13) \\
\mathfrak{s o}(12,1)\end{array}$ & $\mathrm{S}$ \\
\hline
\end{tabular}

Table 100: Maximal subalgebras of $\mathfrak{s o}(3,11)$.

\begin{tabular}{|c|c|c|}
\hline type & real subalgebras & \\
\hline \multirow{4}{*}{$A_{3}+D_{4}$} & $\mathfrak{s l}(4, \mathbb{R}) \oplus \mathfrak{s o}(8)$ & \multirow{4}{*}{$\mathrm{R}$} \\
\hline & $\mathfrak{s u}(2,2) \oplus \mathfrak{s o}(1,7)$ & \\
\hline & $\mathfrak{s u}(4) \oplus \mathfrak{s o}(3,5)$ & \\
\hline & $\mathfrak{s l}(2, \mathbb{H}) \oplus \mathfrak{s o}^{*}(8)$ & \\
\hline \multirow{4}{*}{$A_{1}+A_{1}+D_{5}$} & $\mathfrak{s l}(2, \mathbb{R}) \oplus \mathfrak{s l}(2, \mathbb{R}) \oplus \mathfrak{s o}(9,1)$ & \multirow{4}{*}{$\mathrm{R}$} \\
\hline & $\mathfrak{s l}(2, \mathbb{C}) \oplus \mathfrak{s o}(2,8)$ & \\
\hline & $\mathfrak{s u}(2) \oplus \mathfrak{s u}(2) \oplus \mathfrak{s o}(3,7)$ & \\
\hline & $\mathfrak{s l}(2, \mathbb{C}) \oplus \mathfrak{s o}(10)$ & \\
\hline \multirow{3}{*}{$D_{6}+T_{1}$} & $\mathfrak{s o}(2,10) \oplus \mathfrak{s o}(1,1)$ & \multirow{3}{*}{$\mathrm{R}$} \\
\hline & $\mathfrak{s o}(3,9) \oplus \mathfrak{u}(1)$ & \\
\hline & $\mathfrak{s o}(11,1) \oplus \mathfrak{u}(1)$ & \\
\hline \multirow{2}{*}{$B_{3}+B_{3}$} & $\mathfrak{s o}(7) \oplus \mathfrak{s o}(4,3)$ & \multirow{2}{*}{$\mathrm{S}$} \\
\hline & $\mathfrak{s o}(2,5) \oplus \mathfrak{s o}(6,1)$ & \\
\hline \multirow{4}{*}{$B_{2}+B_{4}$} & $\mathfrak{s o}(5) \oplus \mathfrak{s o}(6,3)$ & \multirow{4}{*}{$\mathrm{S}$} \\
\hline & $\mathfrak{s o}(2,3) \oplus \mathfrak{s o}(9)$ & \\
\hline & $\mathfrak{s o}(2,3) \oplus \mathfrak{s o}(8,1)$ & \\
\hline & $\mathfrak{s o}(4,1) \oplus \mathfrak{s o}(2,7)$ & \\
\hline \multirow{4}{*}{$A_{1}+B_{5}$} & $\mathfrak{s u}(2) \oplus \mathfrak{s o}(11)$ & \multirow{4}{*}{$\mathrm{S}$} \\
\hline & $\mathfrak{s u}(2) \oplus \mathfrak{s o}(8,3)$ & \\
\hline & $\mathfrak{s l}(2, \mathbb{R}) \oplus \mathfrak{s o}(2,9)$ & \\
\hline & $\mathfrak{s l}(2, \mathbb{R}) \oplus \mathfrak{s} \mathfrak{o}(10,1)$ & \\
\hline \multirow{2}{*}{$B_{6}$} & $\mathfrak{s o}(2,11)$ & \multirow{2}{*}{$\mathrm{S}$} \\
\hline & $\mathfrak{s o}(10,3)$ & \\
\hline
\end{tabular}

Table 101: Maximal subalgebras of $\mathfrak{s o}(5,9)$.

\begin{tabular}{|l|c|l|}
\hline type & real subalgebras & \\
\hline & $\mathfrak{s l}(4, \mathbb{R}) \oplus \mathfrak{s o}(8)$ & \\
& $\mathfrak{s u}(2,2) \oplus \mathfrak{s o}(3,5)$ & \\
& $\mathfrak{s l}(2, \mathbb{H}) \oplus \mathfrak{s o}(4,4)$ & \\
$A_{3}+D_{4}$ & $\mathfrak{s u}(2,2) \oplus \mathfrak{s o}(1,7)$ & $\mathrm{R}$ \\
& $\mathfrak{s u}(4) \oplus \mathfrak{s o}(3,5)$ & \\
& $\mathfrak{s l}(2, \mathbb{H}) \oplus \mathfrak{s o}(8)$ & \\
& &
\end{tabular}




\begin{tabular}{|c|c|c|}
\hline \multicolumn{3}{|c|}{ subalgebras of $\mathfrak{s o}(5,9)$} \\
\hline \multirow{5}{*}{$A_{1}+A_{1}+D_{5}$} & $\mathfrak{s l}(2, \mathbb{R}) \oplus \mathfrak{s l}(2, \mathbb{R}) \oplus \mathfrak{s o}(3,7)$ & \\
\hline & $\mathfrak{s l}(2, \mathbb{C}) \oplus \mathfrak{s o}(4,6)$ & \\
\hline & $\mathfrak{s u}(2) \oplus \mathfrak{s u}(2) \oplus \mathfrak{s o}(5,5)$ & $\mathrm{R}$ \\
\hline & $\mathfrak{s l}(2, \mathbb{C}) \oplus \mathfrak{s o}(2,8)$ & \\
\hline & $\mathfrak{s u}(2) \oplus \mathfrak{s u}(2) \oplus \mathfrak{s o}(9,1)$ & \\
\hline \multirow{3}{*}{$D_{6}+T_{1}$} & $\mathfrak{s o}(4,8) \oplus \mathfrak{s o}(1,1)$ & \\
\hline & $\mathfrak{s o}(5,7) \oplus \mathfrak{u}(1)$ & $\mathrm{R}$ \\
\hline & $\mathfrak{s o}(3,9) \oplus \mathfrak{u}(1)$ & \\
\hline \multirow{2}{*}{$B_{3}+B_{3}$} & $\mathfrak{s o}(7) \oplus \mathfrak{s o}(2,5)$ & $\mathrm{S}$ \\
\hline & $\mathfrak{s o}(2,5) \oplus \mathfrak{s o}(4,3)$ & $S$ \\
\hline \multirow{5}{*}{$B_{2}+B_{4}$} & $\mathfrak{s o}(5) \oplus \mathfrak{s o}(9)$ & \\
\hline & $\mathfrak{s o}(5) \oplus \mathfrak{s o}(4,5)$ & \\
\hline & $\mathfrak{s o}(2,3) \oplus \mathfrak{s o}(2,7)$ & $\mathrm{S}$ \\
\hline & $\mathfrak{s o}(2,3) \oplus \mathfrak{s o}(6,3)$ & \\
\hline & $\mathfrak{s o}(4,1) \oplus \mathfrak{s o}(8,1)$ & \\
\hline \multirow{4}{*}{$A_{1}+B_{5}$} & $\mathfrak{s u}(2) \oplus \mathfrak{s o}(2,9)$ & \\
\hline & $\mathfrak{s u}(2) \oplus \mathfrak{s o}(6,5)$ & \\
\hline & $\mathfrak{s l}(2, \mathbb{R}) \oplus \mathfrak{s o}(4,7)$ & $S$ \\
\hline & $\mathfrak{s l}(2, \mathbb{R}) \oplus \mathfrak{s o}(8,3)$ & \\
\hline \multirow{2}{*}{$B_{6}$} & $\mathfrak{s o}(4,9)$ & $\mathrm{S}$ \\
\hline & $\mathfrak{s o}(8,5)$ & $\mathrm{N}$ \\
\hline
\end{tabular}

Table 102: Maximal subalgebras of $\mathfrak{s o}(7,7)$.

\begin{tabular}{|l|c|l|}
\hline type & real subalgebras & \\
\hline$A_{6}+T_{1}$ & $\mathfrak{s l}(7, \mathbb{R}) \oplus \mathfrak{s o}(1,1)$ & $\mathrm{R}$ \\
\hline & $\mathfrak{s l}(4, \mathbb{R}) \oplus \mathfrak{s o}(4,4)$ & \\
$A_{3}+D_{4}$ & $\mathfrak{s u}(2,2) \oplus \mathfrak{s o}(3,5)(2 \times)$ & \\
& $\mathfrak{s l}(2, \mathbb{H}) \oplus \mathfrak{s o}(8)(2 \times)$ & $\mathrm{R}$ \\
& $\mathfrak{s u}(4) \oplus \mathfrak{s o}(1,7)(2 \times)$ & \\
\hline & $\mathfrak{s l}(2, \mathbb{R}) \oplus \mathfrak{s l}(2, \mathbb{R}) \oplus \mathfrak{s o}(5,5)$ & \\
$A_{1}+A_{1}+D_{5}$ & $\mathfrak{s l}(2, \mathbb{C}) \oplus \mathfrak{s o}(4,6)(2 \times)$ & $\mathrm{R}$ \\
& $\mathfrak{s u}(2) \oplus \mathfrak{s u}(2) \oplus \mathfrak{s o}(3,7)(\times)$ & \\
\hline$D_{6}+T_{1}$ & $\mathfrak{s o}(6,6) \oplus \mathfrak{s o}(1,1)$ & $\mathrm{R}$ \\
& $\mathfrak{s o}(5,7) \oplus \mathfrak{u}(1)(2 \times)$ & \\
\hline$B_{3}+B_{3}$ & $\mathfrak{s o}(2,5) \oplus \mathfrak{s o}(2,5)$ & \\
& $\mathfrak{s o}(4,3) \oplus \mathfrak{s o}(4,3)$ & $\mathrm{S}$ \\
& $\mathfrak{s o}(6,1) \oplus \mathfrak{s o}(6,1)$ & \\
\hline \multirow{3}{*}{$B_{2}+B_{4}$} & $\mathfrak{s o}(5) \oplus \mathfrak{s o}(2,7)$ & \\
& $\mathfrak{s o}(2,3) \oplus \mathfrak{s o}(4,5)$ & $\mathrm{S}$ \\
\hline$A_{1}+B_{5}$ & $\mathfrak{s o}(4,1) \oplus \mathfrak{s o}(6,3)$ & \\
\hline$B_{6}$ & $\mathfrak{s u}(2) \oplus \mathfrak{s o}(4,7)$ & $\mathrm{S}$ \\
\hline & $\mathfrak{s l}(2, \mathbb{R}) \oplus \mathfrak{s o}(6,5)$ & $\mathrm{S}(6,7)$ \\
\hline
\end{tabular}


Type $E_{7}$

Table 103: Maximal subalgebras of $E_{7(7)}$.

\begin{tabular}{|l|c|c|}
\hline type & real subalgebras & \\
\hline$E_{6}+T_{1}$ & $\begin{array}{r}E_{6(6)} \oplus \mathfrak{s o}(1,1) \\
E_{6(2)} \oplus \mathfrak{u}(1)\end{array}$ & $\mathrm{R}$ \\
\hline$A_{1}+D_{6}$ & $\begin{array}{r}\mathfrak{s l}(2, \mathbb{R}) \oplus \mathfrak{s o}(6,6) \\
\mathfrak{s u}(2) \oplus \mathfrak{s o}{ }^{*}(12)\end{array}$ & $\mathrm{R}$ \\
\hline & $\mathfrak{s l}(8, \mathbb{R})$ & \\
& $\mathfrak{s u}(4,4)$ & $\mathrm{R}$ \\
$A_{7}$ & $\mathfrak{s l}(4, \mathbb{H})$ & \\
& $\mathfrak{s u}(8)$ & \\
\hline & $\mathfrak{s l}(3, \mathbb{R}) \oplus \mathfrak{s l}(6, \mathbb{R})$ & \\
$A_{2}+A_{5}$ & $\mathfrak{s u}(1,2) \oplus \mathfrak{s u}(3,3)$ & $\mathrm{R}$ \\
& $\mathfrak{s u}(3) \oplus \mathfrak{s u}(1,5)$ & \\
\hline$A_{1}^{231}$ & $\mathfrak{s l}(2, \mathbb{R})(2 \times)$ & $\mathrm{S}$ \\
\hline$A_{1}^{399}$ & $\mathfrak{s l}(2, \mathbb{R})(2 \times)$ & $\mathrm{S}$ \\
\hline$A_{2}$ & $\mathfrak{s l}(3, \mathbb{R})$ & $\mathrm{S}$ \\
\hline$A_{1}+A_{1}$ & $\mathfrak{s l}(2, \mathbb{R}) \oplus \mathfrak{s l}(2, \mathbb{R})$ & $\mathrm{S}$ \\
\hline$A_{1}+G_{2}$ & $\mathfrak{s l}(2, \mathbb{R}) \oplus G_{2}^{c}$ & $\mathrm{~S}$ \\
& $\mathfrak{s l}(2, \mathbb{R}) \oplus G_{2(2)}$ & \\
\hline$C_{3}+G_{2}$ & $\mathfrak{s p}(3, \mathbb{R}) \oplus G_{2(2)}$ & $\mathrm{S}$ \\
\hline$A_{1}+F_{4}$ & $\mathfrak{s l}(2, \mathbb{R}) \oplus F_{4(4)}$ & $\mathrm{S}$ \\
\hline
\end{tabular}

Table 104: Maximal subalgebras of $E_{7(-25)}$.

\begin{tabular}{|c|c|c|}
\hline type & real subalgebras & \\
\hline \multirow{3}{*}{$E_{6}+T_{1}$} & $E_{6(-26)} \oplus \mathfrak{s o}(1,1)$ & \multirow{3}{*}{$\mathrm{R}$} \\
\hline & $E_{6(-14)} \oplus \mathfrak{u}(1)$ & \\
\hline & $E_{6}^{\mathrm{cpt}} \oplus \mathfrak{u}(1)$ & \\
\hline \multirow{2}{*}{$A_{1}+D_{6}$} & $\mathfrak{s l}(2, \mathbb{R}) \oplus \mathfrak{s o}(2,10)$ & \multirow{2}{*}{$\mathrm{R}$} \\
\hline & $\mathfrak{s u}(2) \oplus \mathfrak{s o}^{*}(12)$ & \\
\hline \multirow{2}{*}{$A_{7}$} & $\mathfrak{s l}(4, \mathbb{H})$ & \multirow{2}{*}{$\mathrm{R}$} \\
\hline & $\mathfrak{s u}(2,6)$ & \\
\hline \multirow{2}{*}{$A_{2}+A_{5}$} & $\mathfrak{s u}(3) \oplus \mathfrak{s u}(3,3)$ & \multirow{2}{*}{$\mathrm{R}$} \\
\hline & $\mathfrak{s u}(1,2) \oplus \mathfrak{s u}(1,5)$ & \\
\hline$C_{3}+G_{2}$ & $\mathfrak{s p}(3, \mathbb{R}) \oplus G_{2}^{\mathrm{cpt}}$ & $\mathrm{S}$ \\
\hline$A_{1}+F_{4}$ & $\begin{array}{c}\mathfrak{s l}(2, \mathbb{R}) \oplus F_{4}^{\mathrm{cpt}} \\
\mathfrak{s l}(2, \mathbb{R}) \oplus F_{4(-20)}\end{array}$ & $\mathrm{S}$ \\
\hline
\end{tabular}

Table 105: Maximal subalgebras of $E_{7(-5)}$.

\begin{tabular}{|l|r|l|}
\hline type & real subalgebras & \\
\hline \multirow{2}{*}{$E_{6}+T_{1}$} & $E_{6(2)} \oplus \mathfrak{u}(1)$ & $\mathrm{R}$ \\
& $E_{6(-14)} \oplus \mathfrak{u}(1)$ & \\
\hline
\end{tabular}




\begin{tabular}{|l|c|c|}
\hline \multicolumn{3}{|c|}{ subalgebras of $E_{7(-5)}$} \\
\hline & $\mathfrak{s l}(2, \mathbb{R}) \oplus \mathfrak{s o}{ }^{*}(12)$ & \\
$A_{1}+D_{6}$ & $\mathfrak{s u}(2) \oplus \mathfrak{s o}(4,8)$ & $\mathrm{R}$ \\
& $\mathfrak{s u}(2) \oplus \mathfrak{s o}(12)$ & \\
\hline$A_{7}$ & $\mathfrak{s u}(4,4)$ & $\mathrm{R}$ \\
& $\mathfrak{s u}(2,6)$ & \\
\hline & $\mathfrak{s l}(3, \mathbb{R}) \oplus \mathfrak{s l}(3, \mathbb{H})$ & \\
$A_{2}+A_{5}$ & $\mathfrak{s u}(1,2) \oplus \mathfrak{s u}(2,4)$ & \\
& $\mathfrak{s u}(3) \oplus \mathfrak{s u}(2,4)$ & $\mathrm{R}$ \\
& $\mathfrak{s u}(1,2) \oplus \mathfrak{s u}(6)$ & \\
\hline$A_{2}$ & $\mathfrak{s u}(1,2)$ & $\mathrm{S}$ \\
\hline$A_{1}+A_{1}$ & $\mathfrak{s u}(2) \oplus \mathfrak{s l}(2, \mathbb{R})$ & $\mathrm{S}$ \\
\hline$A_{1}+G_{2}$ & $\mathfrak{s u}(2) \oplus G_{2(2)}$ & $\mathrm{S}$ \\
\hline & $\mathfrak{s p}(3) \oplus G_{2(2)}$ & \\
$C_{3}+G_{2}$ & $\mathfrak{s p}(1,2) \oplus G_{2}^{\mathrm{cpt}}$ & $\mathrm{S}$ \\
& $\mathfrak{s p}(1,2) \oplus G_{2(2)}$ & \\
\hline$A_{1}+F_{4}$ & $\mathfrak{s u}(2) \oplus F_{4(4)}$ & $\mathrm{S}$ \\
& $\mathfrak{s u}(2) \oplus F_{4(-20)}$ & \\
\hline
\end{tabular}

Type $A_{8}$

Table 106: Maximal subalgebras of $\mathfrak{s u}(1,8)$.

\begin{tabular}{|l|c|c|}
\hline type & real subalgebras & \\
\hline$A_{3}+A_{4}+T_{1}$ & $\begin{array}{c}\mathfrak{s u}(1,3) \oplus \mathfrak{s u}(5) \oplus \mathfrak{u}(1) \\
\mathfrak{s u}(4) \oplus \mathfrak{s u}(1,4) \oplus \mathfrak{u}(1)\end{array}$ & $\mathrm{R}$ \\
\hline$A_{2}+A_{5}+T_{1}$ & $\begin{array}{r}\mathfrak{s u}(1,2) \oplus \mathfrak{s u}(6) \oplus \mathfrak{u}(1) \\
\mathfrak{s u}(3) \oplus \mathfrak{s u}(1,5) \oplus \mathfrak{u}(1)\end{array}$ & $\mathrm{R}$ \\
\hline$A_{1}+A_{6}+T_{1}$ & $\begin{array}{c}\mathfrak{s u}(2) \oplus \mathfrak{s u}(1,6) \oplus \mathfrak{u}(1) \\
\mathfrak{s l}(2, \mathbb{R}) \oplus \mathfrak{s u}(7) \oplus \mathfrak{u}(1)\end{array}$ & $\mathrm{R}$ \\
\hline$A_{7}+T_{1}$ & $\mathfrak{s u}(1,7) \oplus \mathfrak{u}(1)$ & $\mathrm{R}$ \\
\hline$B_{4}$ & $\mathfrak{s u}(8) \oplus \mathfrak{u}(1)$ & $\mathrm{S}$ \\
\hline
\end{tabular}

Table 107: Maximal subalgebras of $\mathfrak{s u}(2,7)$.

\begin{tabular}{|l|r|l|}
\hline type & real subalgebras & \\
\hline & $\mathfrak{s u}(2,2) \oplus \mathfrak{s u}(5) \oplus \mathfrak{u}(1)$ & \\
$A_{3}+A_{4}+T_{1}$ & $\mathfrak{s u}(1,3) \oplus \mathfrak{s u}(1,4) \oplus \mathfrak{u}(1)$ & $\mathrm{R}$ \\
& $\mathfrak{s u}(4) \oplus \mathfrak{s u}(2,3) \oplus \mathfrak{u}(1)$ & \\
\hline & $\mathfrak{s u}(1,2) \oplus \mathfrak{s u}(1,5) \oplus \mathfrak{u}(1)$ & \\
$A_{2}+A_{5}+T_{1}$ & $\mathfrak{s u}(3) \oplus \mathfrak{s u}(2,4) \oplus \mathfrak{u}(1)$ & $\mathrm{R}$ \\
& $\mathfrak{s u}(1,2) \oplus \mathfrak{s u}(6) \oplus \mathfrak{u}(1)$ & \\
\hline & $\mathfrak{s u}(2) \oplus \mathfrak{s u}(2,5) \oplus \mathfrak{u}(1)$ & \\
$A_{1}+A_{6}+T_{1}$ & $\mathfrak{s l}(2, \mathbb{R}) \oplus \mathfrak{s u}(1,6) \oplus \mathfrak{u}(1)$ & $\mathrm{R}$ \\
& $\mathfrak{s u}(2) \oplus \mathfrak{s u}(7) \oplus \mathfrak{u}(1)$ & \\
\hline$A_{7}+T_{1}$ & $\mathfrak{s u}(2,6) \oplus \mathfrak{u}(1)$ & $\mathrm{R}$ \\
\hline
\end{tabular}




\begin{tabular}{|l|l|l|}
\hline$B_{4}$ & $\mathfrak{s o}(2,7)$ & $\mathrm{S}$ \\
\hline
\end{tabular}

Table 108: Maximal subalgebras of $\mathfrak{s u}(3,6)$.

\begin{tabular}{|l|r|l|}
\hline type & real subalgebras & \\
\hline & $\mathfrak{s u}(2,2) \oplus \mathfrak{s u}(1,4) \oplus \mathfrak{u}(1)$ & \\
$A_{3}+A_{4}+T_{1}$ & $\mathfrak{s u}(1,3) \oplus \mathfrak{s u}(2,3) \oplus \mathfrak{u}(1)$ & \\
& $\mathfrak{s u}(4) \oplus \mathfrak{s u}(2,3) \oplus \mathfrak{u}(1)$ & $\mathrm{R}$ \\
& $\mathfrak{s u}(1,3) \oplus \mathfrak{s u}(5) \oplus \mathfrak{u}(1)$ & \\
\hline & $\mathfrak{s u}(1,2) \oplus \mathfrak{s u}(2,4) \oplus \mathfrak{u}(1)$ & \\
& $\mathfrak{s u}(3) \oplus \mathfrak{s u}(3,3) \oplus \mathfrak{u}(1)$ & $\mathrm{R}$ \\
$A_{2}+A_{5}+T_{1}$ & $\mathfrak{s u}(1,2) \oplus \mathfrak{s u}(1,5) \oplus \mathfrak{u}(1)$ & \\
& $\mathfrak{s u}(3) \oplus \mathfrak{s u}(6) \oplus \mathfrak{u}(1)$ & \\
\hline & $\mathfrak{s u}(2) \oplus \mathfrak{s u}(3,4) \oplus \mathfrak{u}(1)$ & \\
$A_{1}+A_{6}+T_{1}$ & $\mathfrak{s l}(2, \mathbb{R}) \oplus \mathfrak{s u}(2,5) \oplus \mathfrak{u}(1)$ & $\mathrm{R}$ \\
& $\mathfrak{s u}(2) \oplus \mathfrak{s u}(1,6) \oplus \mathfrak{u}(1)$ & \\
\hline$A_{7}+T_{1}$ & $\mathfrak{s u}(2,6) \oplus \mathfrak{u}(1)$ & $\mathrm{R}$ \\
\hline$A_{2}+A_{2}$ & $\mathfrak{s u}(3,5) \oplus \mathfrak{u}(1)$ & \\
\hline$B_{4}$ & $\mathfrak{s u}(3) \oplus \mathfrak{s u}(1,2)$ & $\mathrm{S}$ \\
\hline
\end{tabular}

Table 109: Maximal subalgebras of $\mathfrak{s u}(4,5)$.

\begin{tabular}{|c|c|c|}
\hline type & real subalgebras & \\
\hline \multirow{5}{*}{$A_{3}+A_{4}+T_{1}$} & $\mathfrak{s u}(2,2) \oplus \mathfrak{s u}(2,3) \oplus \mathfrak{u}(1)$ & \multirow{5}{*}{$\mathrm{R}$} \\
\hline & $\mathfrak{s u}(1,3) \oplus \mathfrak{s u}(2,3) \oplus \mathfrak{u}(1)$ & \\
\hline & $\mathfrak{s u}(4) \oplus \mathfrak{s u}(1,4) \oplus \mathfrak{u}(1)$ & \\
\hline & $\mathfrak{s u}(1,3) \oplus \mathfrak{s u}(1,4) \oplus \mathfrak{u}(1)$ & \\
\hline & $\mathfrak{s u}(4) \oplus \mathfrak{s u}(5) \oplus \mathfrak{u}(1)$ & \\
\hline \multirow{4}{*}{$A_{2}+A_{5}+T_{1}$} & $\mathfrak{s u}(1,2) \oplus \mathfrak{s u}(3,3) \oplus \mathfrak{u}(1)$ & \multirow{4}{*}{$\mathrm{R}$} \\
\hline & $\mathfrak{s u}(1,2) \oplus \mathfrak{s u}(2,4) \oplus \mathfrak{u}(1)$ & \\
\hline & $\mathfrak{s u}(3) \oplus \mathfrak{s u}(2,4) \oplus \mathfrak{u}(1)$ & \\
\hline & $\mathfrak{s u}(3) \oplus \mathfrak{s u}(1,5) \oplus \mathfrak{u}(1)$ & \\
\hline \multirow{3}{*}{$A_{1}+A_{6}+T_{1}$} & $\mathfrak{s l}(2, \mathbb{R}) \oplus \mathfrak{s u}(3,4) \oplus \mathfrak{u}(1)$ & \multirow{3}{*}{$\mathrm{R}$} \\
\hline & $\mathfrak{s u}(2) \oplus \mathfrak{s u}(3,4) \oplus \mathfrak{u}(1)$ & \\
\hline & $\mathfrak{s u}(2) \oplus \mathfrak{s u}(2,5) \oplus \mathfrak{u}(1)$ & \\
\hline \multirow{2}{*}{$A_{7}+T_{1}$} & $\mathfrak{s u}(4,4) \oplus \mathfrak{u}(1)$ & \multirow{2}{*}{$\mathrm{R}$} \\
\hline & $\mathfrak{s u}(3,5) \oplus \mathfrak{u}(1)$ & \\
\hline$A_{2}+A_{2}$ & $\mathfrak{s u}(1,2) \oplus \mathfrak{s u}(1,2)$ & $\mathrm{S}$ \\
\hline$B_{4}$ & $\mathfrak{s o}(4,5)$ & $\mathrm{S}$ \\
\hline
\end{tabular}

Table 110: Maximal subalgebras of $\mathfrak{s l}(9, \mathbb{R})$.

\begin{tabular}{|l|c|c|}
\hline type & real subalgebras & \\
\hline$A_{3}+A_{4}+T_{1}$ & $\mathfrak{s l}(4, \mathbb{R}) \oplus \mathfrak{s l}(5, \mathbb{R}) \oplus \mathfrak{s o}(1,1)$ & $\mathrm{R}$ \\
\hline$A_{2}+A_{5}+T_{1}$ & $\mathfrak{s l}(3, \mathbb{R}) \oplus \mathfrak{s l}(6, \mathbb{R}) \oplus \mathfrak{s o}(1,1)$ & $\mathrm{R}$ \\
\hline
\end{tabular}




\begin{tabular}{|l|c|c|}
\hline \multicolumn{3}{|l|}{ subalgebras of $\mathfrak{s l}(9, \mathbb{R})$} \\
\hline$A_{1}+A_{6}+T_{1}$ & $\mathfrak{s l}(2, \mathbb{R}) \oplus \mathfrak{s l}(7, \mathbb{R}) \oplus \mathfrak{s o}(1,1)$ & $\mathrm{R}$ \\
\hline$A_{7}+T_{1}$ & $\mathfrak{s l}(8, \mathbb{R}) \oplus \mathfrak{s o}(1,1)$ & $\mathrm{R}$ \\
\hline$A_{2}+A_{2}$ & $\mathfrak{s l}(3, \mathbb{R}) \oplus \mathfrak{s l}(3, \mathbb{R})$ & $\mathrm{S}$ \\
\hline & $\mathfrak{s o}(9)$ & \\
& $\mathfrak{s o}(2,7)$ & \\
$B_{4}$ & $\mathfrak{s o}(4,5)$ & $\mathrm{S}$ \\
& $\mathfrak{s o}(6,3)$ & \\
& $\mathfrak{s o}(8,1)$ & \\
\hline
\end{tabular}

Type $B_{8}$

Table 111: Maximal subalgebras of $\mathfrak{s o}(2,15)$.

\begin{tabular}{|c|c|c|}
\hline type & real subalgebras & \\
\hline \multirow{3}{*}{$2 A_{1}+B_{6}$} & $\mathfrak{s l}(2, \mathbb{R}) \oplus \mathfrak{s l}(2, \mathbb{R}) \oplus \mathfrak{s o}(13)$ & \\
\hline & $\mathfrak{s l}(2, \mathbb{C}) \oplus \mathfrak{s o}(12,1)$ & $\mathrm{R}$ \\
\hline & $\mathfrak{s u}(2) \oplus \mathfrak{s u}(2) \oplus \mathfrak{s o}(2,11)$ & \\
\hline \multirow{3}{*}{$A_{3}+B_{5}$} & $\mathfrak{s u}(2,2) \oplus \mathfrak{s o}(11)$ & \\
\hline & $\mathfrak{s l}(2, \mathbb{H}) \oplus \mathfrak{s o}(10,1)$ & $\mathrm{R}$ \\
\hline & $\mathfrak{s u}(4) \oplus \mathfrak{s o}(2,9)$ & \\
\hline \multirow{3}{*}{$B_{4}+D_{4}$} & $\mathfrak{s o}(9) \oplus \mathfrak{s o}^{*}(8)$ & \\
\hline & $\mathfrak{s o}(8,1) \oplus \mathfrak{s o}(1,7)$ & $\mathrm{R}$ \\
\hline & $\mathfrak{s o}(2,7) \oplus \mathfrak{s o}(8)$ & \\
\hline \multirow{3}{*}{$B_{3}+D_{5}$} & $\mathfrak{s o}(7) \oplus \mathfrak{s o}(2,8)$ & \\
\hline & $\mathfrak{s o}(6,1) \oplus \mathfrak{s o}(9,1)$ & $\mathrm{R}$ \\
\hline & $\mathfrak{s o}(2,5) \oplus \mathfrak{s o}(10)$ & \\
\hline \multirow{3}{*}{$B_{2}+D_{6}$} & $\mathfrak{s o}(5) \oplus \mathfrak{s o}(2,10)$ & \\
\hline & $\mathfrak{s o}(4,1) \oplus \mathfrak{s o}(11,1)$ & $\mathrm{R}$ \\
\hline & $\mathfrak{s o}(2,3) \oplus \mathfrak{s o}(12)$ & \\
\hline \multirow{3}{*}{$A_{1}+D_{7}$} & $\mathfrak{s u}(2) \oplus \mathfrak{s o}(2,12)$ & \\
\hline & $\mathfrak{s l}(2, \mathbb{R}) \oplus \mathfrak{s o}(13,1)$ & $\mathrm{R}$ \\
\hline & $\mathfrak{s l}(2, \mathbb{R}) \oplus \mathfrak{s o}(14)$ & \\
\hline \multirow{2}{*}{$D_{8}$} & $\mathfrak{s o}(2,14)$ & \\
\hline & $\mathfrak{s o}(15,1)$ & $\mathrm{R}$ \\
\hline \multirow{3}{*}{$B_{7}+T_{1}$} & $\mathfrak{s o}(14,1) \oplus \mathfrak{s o}(1,1)$ & \\
\hline & $\mathfrak{s o}(2,13) \oplus \mathfrak{u}(1)$ & $\mathrm{R}$ \\
\hline & $\mathfrak{s o}(15) \oplus \mathfrak{u}(1)$ & \\
\hline
\end{tabular}

Table 112: Maximal subalgebras of $\mathfrak{s o}(4,13)$.

\begin{tabular}{|l|r|l|}
\hline type & real subalgebras & \\
\hline & $\mathfrak{s l}(2, \mathbb{R}) \oplus \mathfrak{s l}(2, \mathbb{R}) \oplus \mathfrak{s o}(2,11)$ & \\
& $\mathfrak{s l}(2, \mathbb{C}) \oplus \mathfrak{s o}(10,3)$ & \\
$2 A_{1}+B_{6}$ & $\mathfrak{s u}(2) \oplus \mathfrak{s u}(2) \oplus \mathfrak{s o}(4,9)$ & $\mathrm{R}$ \\
& $\mathfrak{s l}(2, \mathbb{C}) \oplus \mathfrak{s o}(12,1)$ & \\
& $\mathfrak{s u}(2) \oplus \mathfrak{s u}(2) \oplus \mathfrak{s o}(13)$ & \\
\hline
\end{tabular}




\begin{tabular}{|c|c|c|}
\hline \multicolumn{3}{|c|}{ subalgebras of $\mathfrak{s o}(4,13)$} \\
\hline \multirow{5}{*}{$A_{3}+B_{5}$} & $\mathfrak{s l}(4, \mathbb{R}) \oplus \mathfrak{s o}(10,1)$ & \multirow{5}{*}{$\mathrm{R}$} \\
\hline & $\mathfrak{s u}(2,2) \oplus \mathfrak{s o}(2,9)$ & \\
\hline & $\mathfrak{s l}(2, \mathbb{H}) \oplus \mathfrak{s o}(8,3)$ & \\
\hline & $\mathfrak{s u}(4) \oplus \mathfrak{s o}(4,7)$ & \\
\hline & $\mathfrak{s u}(2,2) \oplus \mathfrak{s o}(11)$ & \\
\hline \multirow{5}{*}{$B_{4}+D_{4}$} & $\mathfrak{s o}(9) \oplus \mathfrak{s o}(4,4)$ & \multirow{5}{*}{$\mathrm{R}$} \\
\hline & $\mathfrak{s o}(8,1) \oplus \mathfrak{s o}(3,5)$ & \\
\hline & $\mathfrak{s o}(2,7) \oplus \mathfrak{s o}^{*}(8)$ & \\
\hline & $\mathfrak{s o}(4,5) \oplus \mathfrak{s o}(8)$ & \\
\hline & $\mathfrak{s o}(6,3) \oplus \mathfrak{s o}(1,7)$ & \\
\hline \multirow{5}{*}{$B_{3}+D_{5}$} & $\mathfrak{s o}(7) \oplus \mathfrak{s o}(4,6)$ & \multirow{5}{*}{$\mathrm{R}$} \\
\hline & $\mathfrak{s o}(6,1) \oplus \mathfrak{s o}(3,7)$ & \\
\hline & $\mathfrak{s o}(2,5) \oplus \mathfrak{s o}(2,8)$ & \\
\hline & $\mathfrak{s o}(4,3) \oplus \mathfrak{s o}(9,1)$ & \\
\hline & $\mathfrak{s o}(4,3) \oplus \mathfrak{s o}(10)$ & \\
\hline \multirow{5}{*}{$B_{2}+D_{6}$} & $\mathfrak{s o}(5) \oplus \mathfrak{s o}(4,8)$ & \multirow{5}{*}{$\mathrm{R}$} \\
\hline & $\mathfrak{s o}(4,1) \oplus \mathfrak{s o}(3,9)$ & \\
\hline & $\mathfrak{s o}(2,3) \oplus \mathfrak{s o}(2,10)$ & \\
\hline & $\mathfrak{s o}(2,3) \oplus \mathfrak{s o}(11,1)$ & \\
\hline & $\mathfrak{s o}(4,1) \oplus \mathfrak{s o}(12)$ & \\
\hline \multirow{4}{*}{$A_{1}+D_{7}$} & $\mathfrak{s u}(2) \oplus \mathfrak{s o}(4,10)$ & \multirow{4}{*}{$\mathrm{R}$} \\
\hline & $\mathfrak{s l}(2, \mathbb{R}) \oplus \mathfrak{s o}(3,11)$ & \\
\hline & $\mathfrak{s l}(2, \mathbb{R}) \oplus \mathfrak{s o}(2,12)$ & \\
\hline & $\mathfrak{s u}(2) \oplus \mathfrak{s o}(13,1)$ & \\
\hline \multirow{2}{*}{$D_{8}$} & $\mathfrak{s o}(4,12)$ & \multirow{2}{*}{$\mathrm{R}$} \\
\hline & $\mathfrak{s o}(3,13)$ & \\
\hline \multirow{3}{*}{$B_{7}+T_{1}$} & $\mathfrak{s o}(12,3) \oplus \mathfrak{s o}(1,1)$ & \multirow{3}{*}{$\mathrm{R}$} \\
\hline & $\mathfrak{s o}(4,11) \oplus \mathfrak{u}(1)$ & \\
\hline & $\mathfrak{s o}(2,13) \oplus \mathfrak{u}(1)$ & \\
\hline
\end{tabular}

Table 113: Maximal subalgebras of $\mathfrak{s o}(6,11)$.

\begin{tabular}{|l|r|r|}
\hline type & real subalgebras & \\
\hline & $\mathfrak{s l}(2, \mathbb{R}) \oplus \mathfrak{s l}(2, \mathbb{R}) \oplus \mathfrak{s o}(4,9)$ & \\
& $\mathfrak{s l}(2, \mathbb{C}) \oplus \mathfrak{s o}(8,5)$ & \\
$2 A_{1}+B_{6}$ & $\mathfrak{s u}(2) \oplus \mathfrak{s u}(2) \oplus \mathfrak{s o}(6,7)$ & $\mathrm{R}$ \\
& $\mathfrak{s l}(2, \mathbb{C}) \oplus \mathfrak{s o}(10,3)$ & \\
& $\mathfrak{s u}(2) \oplus \mathfrak{s u}(2) \oplus \mathfrak{s o}(2,11)$ & \\
\hline
\end{tabular}




\begin{tabular}{|c|c|c|}
\hline \multirow{7}{*}{$A_{3}+B_{5}$} & $\mathfrak{s l}(4, \mathbb{R}) \oplus \mathfrak{s o}(8,3)$ & \multirow{7}{*}{$\mathrm{R}$} \\
\hline & $\mathfrak{s u}(2,2) \oplus \mathfrak{s o}(4,7)$ & \\
\hline & $\mathfrak{s l}(2, \mathbb{H}) \oplus \mathfrak{s o}(6,5)$ & \\
\hline & $\mathfrak{s u}(4) \oplus \mathfrak{s o}(6,5)$ & \\
\hline & $\mathfrak{s u}(2,2) \oplus \mathfrak{s o}(2,9)$ & \\
\hline & $\mathfrak{s l}(2, \mathbb{H}) \oplus \mathfrak{s o}(10,1)$ & \\
\hline & $\mathfrak{s u}(4) \oplus \mathfrak{s o}(11)$ & \\
\hline \multirow{7}{*}{$B_{4}+D_{4}$} & $\mathfrak{s o}(2,7) \oplus \mathfrak{s o}(4,4)$ & \multirow{7}{*}{$\mathrm{R}$} \\
\hline & $\mathfrak{s o}(6,3) \oplus \mathfrak{s o}(3,5)$ & \\
\hline & $\mathfrak{s o}(4,5) \oplus \mathfrak{s o}^{*}(8)$ & \\
\hline & $\mathfrak{s o}(4,5) \oplus \mathfrak{s o}(1,7)$ & \\
\hline & $\mathfrak{s o}(8,1) \oplus \mathfrak{s o}(3,5)$ & \\
\hline & $\mathfrak{s o}(6,3) \oplus \mathfrak{s o}(8)$ & \\
\hline & $\mathfrak{s o}(9) \oplus \mathfrak{s o}^{*}(8)$ & \\
\hline \multirow{7}{*}{$B_{3}+D_{5}$} & $\mathfrak{s o}(6,1) \oplus \mathfrak{s o}(5,5)$ & \multirow{7}{*}{$\mathrm{R}$} \\
\hline & $\mathfrak{s o}(2,5) \oplus \mathfrak{s o}(4,6)$ & \\
\hline & $\mathfrak{s o}(4,3) \oplus \mathfrak{s o}(3,7)$ & \\
\hline & $\mathfrak{s o}(4,3) \oplus \mathfrak{s o}(2,8)$ & \\
\hline & $\mathfrak{s o}(7) \oplus \mathfrak{s o}(4,6)$ & \\
\hline & $\mathfrak{s o}(2,5) \oplus \mathfrak{s o}(9,1)$ & \\
\hline & $\mathfrak{s o}(6,1) \oplus \mathfrak{s o}(10)$ & \\
\hline \multirow{6}{*}{$B_{2}+D_{6}$} & $\mathfrak{s o}(5) \oplus \mathfrak{s o}(6,6)$ & \multirow{6}{*}{$\mathrm{R}$} \\
\hline & $\mathfrak{s o}(4,1) \oplus \mathfrak{s o}(5,7)$ & \\
\hline & $\mathfrak{s o}(2,3) \oplus \mathfrak{s o}(4,8)$ & \\
\hline & $\mathfrak{s o}(2,3) \oplus \mathfrak{s o}(3,9)$ & \\
\hline & $\mathfrak{s o}(4,1) \oplus \mathfrak{s o}(2,10)$ & \\
\hline & $\mathfrak{s o}(5) \oplus \mathfrak{s o}(11,1)$ & \\
\hline \multirow{4}{*}{$A_{1}+D_{7}$} & $\mathfrak{s u}(2) \oplus \mathfrak{s o}(6,8)$ & \multirow{4}{*}{$\mathrm{R}$} \\
\hline & $\mathfrak{s l}(2, \mathbb{R}) \oplus \mathfrak{s} \mathfrak{o}(5,9)$ & \\
\hline & $\mathfrak{s l}(2, \mathbb{R}) \oplus \mathfrak{s o}(4,10)$ & \\
\hline & $\mathfrak{s u}(2) \oplus \mathfrak{s o}(3,11)$ & \\
\hline \multirow{2}{*}{$D_{8}$} & $\mathfrak{s o}(6,10)$ & \multirow{2}{*}{$\mathrm{R}$} \\
\hline & $\mathfrak{s o}(5,11)$ & \\
\hline \multirow{3}{*}{$B_{7}+T_{1}$} & $\mathfrak{s o}(10,5) \oplus \mathfrak{s o}(1,1)$ & \multirow{3}{*}{$\mathrm{R}$} \\
\hline & $\mathfrak{s o}(6,9) \oplus \mathfrak{u}(1)$ & \\
\hline & $\mathfrak{s o}(4,11) \oplus \mathfrak{u}(1)$ & \\
\hline
\end{tabular}

Table 114: Maximal subalgebras of $\mathfrak{s o}(8,9)$. 


\begin{tabular}{|c|c|c|}
\hline \multicolumn{3}{|c|}{ subalgebras of $\mathfrak{s o}(8,9)$} \\
\hline \multirow{5}{*}{$2 A_{1}+B_{6}$} & $\mathfrak{s l}(2, \mathbb{R}) \oplus \mathfrak{s l}(2, \mathbb{R}) \oplus \mathfrak{s o}(6,7)$ & \multirow{5}{*}{$\mathrm{R}$} \\
\hline & $\mathfrak{s l}(2, \mathbb{C}) \oplus \mathfrak{s o}(6,7)$ & \\
\hline & $\mathfrak{s l}(2, \mathbb{C}) \oplus \mathfrak{s o}(8,5)$ & \\
\hline & $\mathfrak{s u}(2) \oplus \mathfrak{s u}(2) \oplus \mathfrak{s o}(8,5)$ & \\
\hline & $\mathfrak{s u}(2) \oplus \mathfrak{s u}(2) \oplus \mathfrak{s o}(4,9)$ & \\
\hline \multirow{7}{*}{$A_{3}+B_{5}$} & $\mathfrak{s l}(4, \mathbb{R}) \oplus \mathfrak{s o}(6,5)$ & \multirow{7}{*}{$\mathrm{R}$} \\
\hline & $\mathfrak{s u}(2,2) \oplus \mathfrak{s o}(6,5)$ & \\
\hline & $\mathfrak{s u}(2,2) \oplus \mathfrak{s o}(4,7)$ & \\
\hline & $\mathfrak{s l}(2, \mathbb{H}) \oplus \mathfrak{s o}(4,7)$ & \\
\hline & $\mathfrak{s l}(2, \mathbb{H}) \oplus \mathfrak{s o}(8,3)$ & \\
\hline & $\mathfrak{s u}(4) \oplus \mathfrak{s o}(8,3)$ & \\
\hline & $\mathfrak{s u}(4) \oplus \mathfrak{s o}(2,9)$ & \\
\hline \multirow{9}{*}{$B_{4}+D_{4}$} & $\mathfrak{s o}(4,5) \oplus \mathfrak{s o}(4,4)$ & \multirow{9}{*}{$\mathrm{R}$} \\
\hline & $\mathfrak{s o}(4,5) \oplus \mathfrak{s o}(3,5)$ & \\
\hline & $\mathfrak{s o}(6,3) \oplus \mathfrak{s o}(3,5)$ & \\
\hline & $\mathfrak{s o}(6,3) \oplus \mathfrak{s o}^{*}(8)$ & \\
\hline & $\mathfrak{s o}(2,7) \oplus \mathfrak{s o}^{*}(8)$ & \\
\hline & $\mathfrak{s o}(2,7) \oplus \mathfrak{s o}(1,7)$ & \\
\hline & $\mathfrak{s o}(8,1) \oplus \mathfrak{s o}(1,7)$ & \\
\hline & $\mathfrak{s o}(8,1) \oplus \mathfrak{s o}(8)$ & \\
\hline & $\mathfrak{s o}(9) \oplus \mathfrak{s o}(8)$ & \\
\hline \multirow{8}{*}{$B_{3}+D_{5}$} & $\mathfrak{s o}(4,3) \oplus \mathfrak{s o}(5,5)$ & \multirow{8}{*}{$\mathrm{R}$} \\
\hline & $\mathfrak{s o}(4,3) \oplus \mathfrak{s o}(4,6)$ & \\
\hline & $\mathfrak{s o}(2,5) \oplus \mathfrak{s o}(4,6)$ & \\
\hline & $\mathfrak{s o}(2,5) \oplus \mathfrak{s o}(3,7)$ & \\
\hline & $\mathfrak{s o}(6,1) \oplus \mathfrak{s o}(3,7)$ & \\
\hline & $\mathfrak{s o}(6,1) \oplus \mathfrak{s o}(2,8)$ & \\
\hline & $\mathfrak{s o}(7) \oplus \mathfrak{s o}(2,8)$ & \\
\hline & $\mathfrak{s o}(7) \oplus \mathfrak{s o}(9,1)$ & \\
\hline \multirow{6}{*}{$B_{2}+D_{6}$} & $\mathfrak{s o}(2,3) \oplus \mathfrak{s o}(6,6)$ & \multirow{6}{*}{$\mathrm{R}$} \\
\hline & $\mathfrak{s o}(2,3) \oplus \mathfrak{s o}(5,7)$ & \\
\hline & $\mathfrak{s o}(4,1) \oplus \mathfrak{s o}(5,7)$ & \\
\hline & $\mathfrak{s o}(4,1) \oplus \mathfrak{s o}(4,8)$ & \\
\hline & $\mathfrak{s o}(5) \oplus \mathfrak{s o}(4,8)$ & \\
\hline & $\mathfrak{s o}(5) \oplus \mathfrak{s o}(3,9)$ & \\
\hline \multirow{4}{*}{$A_{1}+D_{7}$} & $\mathfrak{s l}(2, \mathbb{R}) \oplus \mathfrak{s o}(7,7)$ & \multirow{4}{*}{$\mathrm{R}$} \\
\hline & $\mathfrak{s l}(2, \mathbb{R}) \oplus \mathfrak{s o}(6,8)$ & \\
\hline & $\mathfrak{s u}(2) \oplus \mathfrak{s o}(6,8)$ & \\
\hline & $\mathfrak{s u}(2) \oplus \mathfrak{s o}(5,9)$ & \\
\hline \multirow{2}{*}{$D_{8}$} & $\mathfrak{s o}(8,8)$ & \multirow{2}{*}{$\mathrm{R}$} \\
\hline & $\mathfrak{s o}(7,9)$ & \\
\hline
\end{tabular}




\begin{tabular}{|l|r|l|}
\hline \multicolumn{2}{|c|}{ subalgebras of $\mathfrak{s o}(8,9)$} \\
\hline \multirow{3}{*}{$B_{7}+T_{1}$} & $\mathfrak{s o}(8,7) \oplus \mathfrak{s o}(1,1)$ & \\
& $\mathfrak{s o}(8,7) \oplus \mathfrak{u}(1)$ & $\mathrm{R}$ \\
& $\mathfrak{s o}(6,9) \oplus \mathfrak{u}(1)$ & \\
\hline$A_{1}$ & $\mathfrak{s l}(2, \mathbb{R})$ & $\mathrm{S}$ \\
\hline
\end{tabular}

Table 115: Maximal subalgebras of $\mathfrak{s o}(10,7)$.

\begin{tabular}{|c|c|c|}
\hline type & real subalgebras & \\
\hline \multirow{5}{*}{$2 A_{1}+B_{6}$} & $\mathfrak{s l}(2, \mathbb{R}) \oplus \mathfrak{s l}(2, \mathbb{R}) \oplus \mathfrak{s o}(8,5)$ & \multirow{5}{*}{$\mathrm{R}$} \\
\hline & $\mathfrak{s l}(2, \mathbb{C}) \oplus \mathfrak{s o}(6,7)$ & \\
\hline & $\mathfrak{s u}(2) \oplus \mathfrak{s u}(2) \oplus \mathfrak{s o}(6,7)$ & \\
\hline & $\mathfrak{s l}(2, \mathbb{C}) \oplus \mathfrak{s o}(4,9)$ & \\
\hline & $\mathfrak{s u}(2) \oplus \mathfrak{s u}(2) \oplus \mathfrak{s o}(10,3)$ & \\
\hline \multirow{7}{*}{$A_{3}+B_{5}$} & $\mathfrak{s l}(4, \mathbb{R}) \oplus \mathfrak{s o}(4,7)$ & \multirow{7}{*}{$\mathrm{R}$} \\
\hline & $\mathfrak{s u}(2,2) \oplus \mathfrak{s o}(6,5)$ & \\
\hline & $\mathfrak{s l}(2, \mathbb{H}) \oplus \mathfrak{s o}(6,5)$ & \\
\hline & $\mathfrak{s u}(2,2) \oplus \mathfrak{s o}(8,3)$ & \\
\hline & $\mathfrak{s u}(4) \oplus \mathfrak{s o}(4,7)$ & \\
\hline & $\mathfrak{s l}(2, \mathbb{H}) \oplus \mathfrak{s o}(2,9)$ & \\
\hline & $\mathfrak{s u}(4) \oplus \mathfrak{s o}(10,1)$ & \\
\hline \multirow{8}{*}{$B_{4}+D_{4}$} & $\mathfrak{s o}(6,3) \oplus \mathfrak{s o}(4,4)$ & \multirow{8}{*}{$\mathrm{R}$} \\
\hline & $\mathfrak{s o}(4,5) \oplus \mathfrak{s o}(3,5)$ & \\
\hline & $\mathfrak{s o}(4,5) \oplus \mathfrak{s o}^{*}(8)$ & \\
\hline & $\mathfrak{s o}(2,7) \oplus \mathfrak{s o}(3,5)$ & \\
\hline & $\mathfrak{s o}(6,3) \oplus \mathfrak{s o}(1,7)$ & \\
\hline & $\mathfrak{s o}(8,1) \oplus \mathfrak{s o}^{*}(8)$ & \\
\hline & $\mathfrak{s o}(2,7) \oplus \mathfrak{s o}(8)$ & \\
\hline & $\mathfrak{s o}(9) \oplus \mathfrak{s o}(1,7)$ & \\
\hline \multirow{8}{*}{$B_{3}+D_{5}$} & $\mathfrak{s o}(2,5) \oplus \mathfrak{s o}(5,5)$ & \multirow{8}{*}{$\mathrm{R}$} \\
\hline & $\mathfrak{s o}(4,3) \oplus \mathfrak{s o}(4,6)$ & \\
\hline & $\mathfrak{s o}(4,3) \oplus \mathfrak{s o}(3,7)$ & \\
\hline & $\mathfrak{s o}(6,1) \oplus \mathfrak{s o}(4,6)$ & \\
\hline & $\mathfrak{s o}(2,5) \oplus \mathfrak{s o}(2,8)$ & \\
\hline & $\mathfrak{s o}(7) \oplus \mathfrak{s o}(3,7)$ & \\
\hline & $\mathfrak{s o}(6,1) \oplus \mathfrak{s o}(9,1)$ & \\
\hline & $\mathfrak{s o}(7) \oplus \mathfrak{s o}(10)$ & \\
\hline \multirow{6}{*}{$B_{2}+D_{6}$} & $\mathfrak{s o}(4,1) \oplus \mathfrak{s o}(6,6)$ & \multirow{6}{*}{$\mathrm{R}$} \\
\hline & $\mathfrak{s o}(2,3) \oplus \mathfrak{s o}(5,7)$ & \\
\hline & $\mathfrak{s o}(2,3) \oplus \mathfrak{s o}(4,8)$ & \\
\hline & $\mathfrak{s o}(5) \oplus \mathfrak{s o}(5,7)$ & \\
\hline & $\mathfrak{s o}(4,1) \oplus \mathfrak{s o}(3,9)$ & \\
\hline & $\mathfrak{s o}(5) \oplus \mathfrak{s o}(2,10)$ & \\
\hline
\end{tabular}




\begin{tabular}{|l|r|l|}
\hline \multicolumn{2}{|c|}{ subalgebras of $\mathfrak{s o}(10,7)$} \\
\hline \multirow{4}{*}{$A_{1}+D_{7}$} & $\mathfrak{s u}(2) \oplus \mathfrak{s o}(7,7)$ & \\
& $\mathfrak{s l}(2, \mathbb{R}) \oplus \mathfrak{s o}(6,8)$ & $\mathrm{R}$ \\
& $\mathfrak{s l}(2, \mathbb{R}) \oplus \mathfrak{s o}(5,9)$ & \\
& $\mathfrak{s u}(2) \oplus \mathfrak{s o}(4,10)$ & \\
\hline$D_{8}$ & $\mathfrak{s o}(7,9)$ & $\mathrm{R}$ \\
\hline \multirow{3}{*}{$B_{7}+T_{1}$} & $\mathfrak{s o}(6,10)$ & \\
& $\mathfrak{s o}(6,9) \oplus \mathfrak{s o}(1,1)$ & $\mathrm{R}$ \\
& $\mathfrak{s o}(8,7) \oplus \mathfrak{u}(1)$ & \\
\hline
\end{tabular}

Table 116: Maximal subalgebras of $\mathfrak{s o}(12,5)$.

\begin{tabular}{|c|c|c|}
\hline type & real subalgebras & \\
\hline \multirow{5}{*}{$2 A_{1}+B_{6}$} & $\mathfrak{s l}(2, \mathbb{R}) \oplus \mathfrak{s l}(2, \mathbb{R}) \oplus \mathfrak{s o}(10,3)$ & \multirow{5}{*}{$\mathrm{R}$} \\
\hline & $\mathfrak{s l}(2, \mathbb{C}) \oplus \mathfrak{s o}(4,9)$ & \\
\hline & $\mathfrak{s u}(2) \oplus \mathfrak{s u}(2) \oplus \mathfrak{s o}(8,5)$ & \\
\hline & $\mathfrak{s l}(2, \mathbb{C}) \oplus \mathfrak{s o}(2,11)$ & \\
\hline & $\mathfrak{s u}(2) \oplus \mathfrak{s u}(2) \oplus \mathfrak{s o}(12,1)$ & \\
\hline \multirow{6}{*}{$A_{3}+B_{5}$} & $\mathfrak{s l}(4, \mathbb{R}) \oplus \mathfrak{s o}(2,9)$ & \multirow{6}{*}{$\mathrm{R}$} \\
\hline & $\mathfrak{s u}(2,2) \oplus \mathfrak{s o}(8,3)$ & \\
\hline & $\mathfrak{s l}(2, \mathbb{H}) \oplus \mathfrak{s o}(4,7)$ & \\
\hline & $\mathfrak{s u}(4) \oplus \mathfrak{s o}(6,5)$ & \\
\hline & $\mathfrak{s u}(2,2) \oplus \mathfrak{s o}(10,1)$ & \\
\hline & $\mathfrak{s l}(2, \mathbb{H}) \oplus \mathfrak{s o}(11)$ & \\
\hline \multirow{6}{*}{$B_{4}+D_{4}$} & $\mathfrak{s o}(8,1) \oplus \mathfrak{s o}(4,4)$ & \multirow{6}{*}{$\mathrm{R}$} \\
\hline & $\mathfrak{s o}(2,7) \oplus \mathfrak{s o}(3,5)$ & \\
\hline & $\mathfrak{s o}(6,3) \oplus \mathfrak{s o}^{*}(8)$ & \\
\hline & $\mathfrak{s o}(4,5) \oplus \mathfrak{s o}(1,7)$ & \\
\hline & $\mathfrak{s o}(4,5) \oplus \mathfrak{s o}(8)$ & \\
\hline & $\mathfrak{s o}(9) \oplus \mathfrak{s o}(3,5)$ & \\
\hline \multirow{6}{*}{$B_{3}+D_{5}$} & $\mathfrak{s o}(7) \oplus \mathfrak{s o}(5,5)$ & \multirow{6}{*}{$\mathrm{R}$} \\
\hline & $\mathfrak{s o}(6,1) \oplus \mathfrak{s o}(4,6)$ & \\
\hline & $\mathfrak{s o}(2,5) \oplus \mathfrak{s o}(3,7)$ & \\
\hline & $\mathfrak{s o}(4,3) \oplus \mathfrak{s o}(2,8)$ & \\
\hline & $\mathfrak{s o}(4,3) \oplus \mathfrak{s o}(9,1)$ & \\
\hline & $\mathfrak{s o}(2,5) \oplus \mathfrak{s o}(10)$ & \\
\hline \multirow{6}{*}{$B_{2}+D_{6}$} & $\mathfrak{s o}(5) \oplus \mathfrak{s o}(5,7)$ & \multirow{6}{*}{$\mathrm{R}$} \\
\hline & $\mathfrak{s o}(4,1) \oplus \mathfrak{s o}(4,8)$ & \\
\hline & $\mathfrak{s o}(2,3) \oplus \mathfrak{s o}(3,9)$ & \\
\hline & $\mathfrak{s o}(2,3) \oplus \mathfrak{s o}(2,10)$ & \\
\hline & $\mathfrak{s o}(4,1) \oplus \mathfrak{s o}(11,1)$ & \\
\hline & $\mathfrak{s o}(5) \oplus \mathfrak{s o}(12)$ & \\
\hline
\end{tabular}




\begin{tabular}{|l|r|l|}
\hline \multicolumn{2}{|c|}{ subalgebras of $\mathfrak{s o}(12,5)$} \\
\hline \multirow{4}{*}{$A_{1}+D_{7}$} & $\mathfrak{s u}(2) \oplus \mathfrak{s o}(5,9)$ & \\
& $\mathfrak{s l}(2, \mathbb{R}) \oplus \mathfrak{s o}(4,10)$ & $\mathrm{R}$ \\
& $\mathfrak{s l}(2, \mathbb{R}) \oplus \mathfrak{s o}(3,11)$ & \\
& $\mathfrak{s u}(2) \oplus \mathfrak{s o}(2,12)$ & \\
\hline$D_{8}$ & $\mathfrak{s o}(5,11)$ & $\mathrm{R}$ \\
\hline \multirow{3}{*}{$B_{7}+T_{1}$} & $\mathfrak{s o}(4,12)$ & \\
& $\mathfrak{s o}(4,11) \oplus \mathfrak{s o}(1,1)$ & $\mathrm{R}$ \\
& $\mathfrak{s o}(10,5) \oplus \mathfrak{u}(1)$ & \\
\hline
\end{tabular}

Table 117: Maximal subalgebras of $\mathfrak{s o}(14,3)$.

\begin{tabular}{|c|c|c|}
\hline type & real subalgebras & \\
\hline \multirow{4}{*}{$2 A_{1}+B_{6}$} & $\mathfrak{s l}(2, \mathbb{R}) \oplus \mathfrak{s l}(2, \mathbb{R}) \oplus \mathfrak{s o}(12,1)$ & \multirow{4}{*}{$\mathrm{R}$} \\
\hline & $\mathfrak{s l}(2, \mathbb{C}) \oplus \mathfrak{s o}(2,11)$ & \\
\hline & $\mathfrak{s u}(2) \oplus \mathfrak{s u}(2) \oplus \mathfrak{s o}(10,3)$ & \\
\hline & $\mathfrak{s l}(2, \mathbb{C}) \oplus \mathfrak{s o}(13)$ & \\
\hline \multirow{4}{*}{$A_{3}+B_{5}$} & $\mathfrak{s l}(4, \mathbb{R}) \oplus \mathfrak{s o}(11)$ & \multirow{4}{*}{$\mathrm{R}$} \\
\hline & $\mathfrak{s u}(2,2) \oplus \mathfrak{s o}(10,1)$ & \\
\hline & $\mathfrak{s l}(2, \mathbb{H}) \oplus \mathfrak{s o}(2,9)$ & \\
\hline & $\mathfrak{s u}(4) \oplus \mathfrak{s o}(8,3)$ & \\
\hline \multirow{4}{*}{$B_{4}+D_{4}$} & $\mathfrak{s o}(9) \oplus \mathfrak{s o}(3,5)$ & \multirow{4}{*}{$\mathrm{R}$} \\
\hline & $\mathfrak{s o}(8,1) \oplus \mathfrak{s o}^{*}(8)$ & \\
\hline & $\mathfrak{s o}(6,3) \oplus \mathfrak{s o}(8)$ & \\
\hline & $\mathfrak{s o}(2,7) \oplus \mathfrak{s o}(1,7)$ & \\
\hline \multirow{4}{*}{$B_{3}+D_{5}$} & $\mathfrak{s o}(7) \oplus \mathfrak{s o}(3,7)$ & \multirow{4}{*}{$\mathrm{R}$} \\
\hline & $\mathfrak{s o}(6,1) \oplus \mathfrak{s o}(2,8)$ & \\
\hline & $\mathfrak{s o}(2,5) \oplus \mathfrak{s o}(9,1)$ & \\
\hline & $\mathfrak{s o}(4,3) \oplus \mathfrak{s o}(10)$ & \\
\hline \multirow{4}{*}{$B_{2}+D_{6}$} & $\mathfrak{s o}(5) \oplus \mathfrak{s o}(3,9)$ & \multirow{4}{*}{$\mathrm{R}$} \\
\hline & $\mathfrak{s o}(4,1) \oplus \mathfrak{s o}(2,10)$ & \\
\hline & $\mathfrak{s o}(2,3) \oplus \mathfrak{s o}(11,1)$ & \\
\hline & $\mathfrak{s o}(2,3) \oplus \mathfrak{s o}(12)$ & \\
\hline \multirow{4}{*}{$A_{1}+D_{7}$} & $\mathfrak{s u}(2) \oplus \mathfrak{s o}(3,11)$ & \multirow{4}{*}{$\mathrm{R}$} \\
\hline & $\mathfrak{s l}(2, \mathbb{R}) \oplus \mathfrak{s o}(2,12)$ & \\
\hline & $\mathfrak{s l}(2, \mathbb{R}) \oplus \mathfrak{s o}(13,1)$ & \\
\hline & $\mathfrak{s u}(2) \oplus \mathfrak{s o}(14)$ & \\
\hline \multirow{2}{*}{$D_{8}$} & $\mathfrak{s o}(3,13)$ & \multirow[b]{2}{*}{$\mathrm{R}$} \\
\hline & $\mathfrak{s o}(2,14)$ & \\
\hline \multirow{3}{*}{$B_{7}+T_{1}$} & $\mathfrak{s o}(2,13) \oplus \mathfrak{s o}(1,1)$ & \multirow{3}{*}{$\mathrm{R}$} \\
\hline & $\mathfrak{s o}(12,3) \oplus \mathfrak{u}(1)$ & \\
\hline & $\mathfrak{s o}(14,1) \oplus \mathfrak{u}(1)$ & \\
\hline
\end{tabular}


Table 118: Maximal subalgebras of $\mathfrak{s o}(16,1)$.

\begin{tabular}{|l|c|l|}
\hline type & real subalgebras & \\
\hline $2 A_{1}+B_{6}$ & $\mathfrak{s l}(2, \mathbb{C}) \oplus \mathfrak{s o}(13)$ & $\mathrm{R}$ \\
& $\mathfrak{s u}(2) \oplus \mathfrak{s u}(2) \oplus \mathfrak{s o}(12,1)$ & \\
\hline$A_{3}+B_{5}$ & $\mathfrak{s l}(2, \mathbb{H}) \oplus \mathfrak{s o}(11)$ & $\mathrm{R}$ \\
\hline$B_{4}+D_{4}$ & $\mathfrak{s u}(4) \oplus \mathfrak{s o}(10,1)$ & \\
\hline$B_{3}+D_{5}$ & $\mathfrak{s o}(8,1) \oplus \mathfrak{s o}(1,7)$ & $\mathrm{R}$ \\
\hline$B_{2}+D_{6}$ & $\mathfrak{s o}(7) \oplus \mathfrak{s o}(9,1)$ & $\mathrm{R}$ \\
\hline$A_{1}+D_{7}$ & $\mathfrak{s o}(5) \oplus \mathfrak{s o}(4,1) \oplus \mathfrak{s o}(11,1)$ & $\mathrm{R}$ \\
\hline$D_{8}$ & $\mathfrak{s u}(2) \oplus \mathfrak{s o}(13,1)$ & $\mathrm{R}$ \\
\hline$B_{7}+T_{1}$ & $\mathfrak{s l}(2, \mathbb{R}) \oplus \mathfrak{s o}(14)$ & \\
\hline
\end{tabular}

Type $C_{8}$

Table 119: Maximal subalgebras of $\mathfrak{s p}(1,7)$.

\begin{tabular}{|l|c|c|}
\hline type & real subalgebras & \\
\hline$A_{7}+T_{1}$ & $\mathfrak{s u}(1,7) \oplus \mathfrak{u}(1)$ & $\mathrm{R}$ \\
\hline$C_{4}+C_{4}$ & $\mathfrak{s p}(4) \oplus \mathfrak{s p}(1,3)$ & $\mathrm{R}$ \\
\hline$C_{3}+C_{5}$ & $\begin{array}{r}\mathfrak{s p}(1,2) \oplus \mathfrak{s p}(5) \\
\mathfrak{s p}(3) \oplus \mathfrak{s p}(1,4)\end{array}$ & $\mathrm{R}$ \\
\hline$B_{2}+C_{6}$ & $\begin{array}{r}\mathfrak{s o}(4,1) \oplus \mathfrak{s p}(6) \\
\mathfrak{s o}(5) \oplus \mathfrak{s p}(1,5)\end{array}$ & $\mathrm{R}$ \\
\hline$A_{1}+C_{7}$ & $\begin{array}{r}\mathfrak{s u}(2) \oplus \mathfrak{s p}(1,6) \\
\mathfrak{s u}(2) \oplus \mathfrak{s p}(7)\end{array}$ & $\mathrm{R}$ \\
\hline$A_{1}+D_{4}$ & $\mathfrak{s u}(2) \oplus \mathfrak{s o}(1,7)$ & $\mathrm{S}$ \\
\hline
\end{tabular}

Table 120: Maximal subalgebras of $\mathfrak{s p}(2,6)$.

\begin{tabular}{|l|r|l|}
\hline type & real subalgebras & \\
\hline$A_{7}+T_{1}$ & $\mathfrak{s u}(2,6) \oplus \mathfrak{u}(1)$ & $\mathrm{R}$ \\
\hline$C_{4}+C_{4}$ & $\mathfrak{s p}(4) \oplus \mathfrak{s p}(2,2)$ & $\mathrm{R}$ \\
& $\mathfrak{s p}(1,3) \oplus \mathfrak{s p}(1,3)$ & \\
\hline & $\mathfrak{s p}(1,2) \oplus \mathfrak{s p}(1,4)$ & \\
$C_{3}+C_{5}$ & $\mathfrak{s p}(3) \oplus \mathfrak{s p}(2,3)$ & $\mathrm{R}$ \\
& $\mathfrak{s p}(1,2) \oplus \mathfrak{s p}(5)$ & \\
\hline & $\mathfrak{s o}(4,1) \oplus \mathfrak{s p}(1,5)$ & \\
$B_{2}+C_{6}$ & $\mathfrak{s o}(5) \oplus \mathfrak{s p}(2,4)$ & $\mathrm{R}$ \\
& $\mathfrak{s o}(5) \oplus \mathfrak{s p}(6)$ & \\
\hline \multirow{2}{*}{$A_{1}+C_{7}$} & $\mathfrak{s u}(2) \oplus \mathfrak{s p}(2,5)$ & $\mathrm{R}$ \\
& $\mathfrak{s u}(2) \oplus \mathfrak{s p}(1,6)$ & \\
\hline
\end{tabular}


Table 121: Maximal subalgebras of $\mathfrak{s p}(3,5)$.

\begin{tabular}{|l|r|l|}
\hline type & real subalgebras & \\
\hline$A_{7}+T_{1}$ & $\mathfrak{s u}(3,5) \oplus \mathfrak{u}(1)$ & $\mathrm{R}$ \\
\hline$C_{4}+C_{4}$ & $\mathfrak{s p}(1,3) \oplus \mathfrak{s p}(2,2)$ & $\mathrm{R}$ \\
& $\mathfrak{s p}(4) \oplus \mathfrak{s p}(1,3)$ & \\
\hline & $\mathfrak{s p}(1,2) \oplus \mathfrak{s p}(2,3)$ & \\
$C_{3}+C_{5}$ & $\mathfrak{s p}(1,2) \oplus \mathfrak{s p}(1,4)$ & \\
& $\mathfrak{s p}(3) \oplus \mathfrak{s p}(2,3)$ & $\mathrm{R}$ \\
& $\mathfrak{s p}(3) \oplus \mathfrak{s p}(5)$ & \\
\hline & $\mathfrak{s o}(4,1) \oplus \mathfrak{s p}(2,4)$ & \\
$B_{2}+C_{6}$ & $\mathfrak{s o}(5) \oplus \mathfrak{s p}(3,3)$ & $\mathrm{R}$ \\
& $\mathfrak{s o}(5) \oplus \mathfrak{s p}(1,5)$ & \\
\hline$A_{1}+C_{7}$ & $\mathfrak{s u}(2) \oplus \mathfrak{s p}(3,4)$ & $\mathrm{R}$ \\
\hline$A_{1}+D_{4}$ & $\mathfrak{s u}(2) \oplus \mathfrak{s u}(2) \oplus \mathfrak{s o}(3,5)$ & \\
\hline
\end{tabular}

Table 122: Maximal subalgebras of $\mathfrak{s p}(4,4)$.

\begin{tabular}{|l|c|c|}
\hline type & real subalgebras & \\
\hline$A_{7}+T_{1}$ & $\mathfrak{s l}(4, \mathbb{H}) \oplus \mathfrak{s u}(1,1)$ & $\mathrm{R}$ \\
& $\mathfrak{s u}(4,4) \oplus \mathfrak{u}(1)$ & \\
\hline & $\mathfrak{s p}(2,2) \oplus \mathfrak{s p}(2,2)$ & \\
$C_{4}+C_{4}$ & $\mathfrak{s p}(4, \mathbb{C})$ & \\
& $\mathfrak{s p}(1,3) \oplus \mathfrak{s p}(1,3)$ & $\mathrm{R}$ \\
& $\mathfrak{s p}(4) \oplus \mathfrak{s p}(4)$ & \\
\hline$C_{3}+C_{5}$ & $\mathfrak{s p}(1,2) \oplus \mathfrak{s p}(2,3)(2 \times)$ & $\mathrm{R}$ \\
\hline$B_{2}+C_{6}$ & $\mathfrak{s p}(3) \oplus \mathfrak{s p}(1,4)(2 \times)$ & \\
\hline$A_{1}+C_{7}$ & $\mathfrak{s o}(4,1) \oplus \mathfrak{s p}(2) \oplus \mathfrak{s p}(3,3)$ & $\mathrm{R}$ \\
\hline$B_{2}$ & $\mathfrak{s o}(4,1)$ & $\mathrm{S}$ \\
\hline$A_{1}+D_{4}$ & $\mathfrak{s u}(2) \oplus \mathfrak{s o}(4,4)$ & $\mathrm{S}$ \\
\hline \multicolumn{3}{|c}{} \\
\hline
\end{tabular}

Table 123: Maximal subalgebras of $\mathfrak{s p}(8, \mathbb{R})$.

\begin{tabular}{|l|r|l|}
\hline type & real subalgebras & \\
\hline & $\mathfrak{s l}(8, \mathbb{R}) \oplus \mathfrak{s o}(1,1)$ & \\
& $\mathfrak{s u}(4,4) \oplus \mathfrak{u}(1)$ & \\
$A_{7}+T_{1}$ & $\mathfrak{s u}(3,5) \oplus \mathfrak{u}(1)$ & \\
& $\mathfrak{s u}(2,6) \oplus \mathfrak{u}(1)$ & $\mathrm{R}$ \\
& $\mathfrak{s u}(1,7) \oplus \mathfrak{u}(1)$ & \\
& $\mathfrak{s u}(8) \oplus \mathfrak{u}(1)$ & \\
\hline$C_{4}+C_{4}$ & $\mathfrak{s p}(4, \mathbb{R}) \oplus \mathfrak{s p}(4, \mathbb{R})$ & \\
& $\mathfrak{s p}(4, \mathbb{C})$ & \\
\hline$C_{3}+C_{5}$ & $\mathfrak{s p}(3, \mathbb{R}) \oplus \mathfrak{s p}(5, \mathbb{R})$ & $\mathrm{R}$ \\
\hline
\end{tabular}




\begin{tabular}{|l|c|c|}
\hline \multicolumn{3}{|c|}{ subalgebras of $\mathfrak{s p}(8, \mathbb{R})$} \\
\hline$B_{2}+C_{6}$ & $\mathfrak{s o}(2,3) \oplus \mathfrak{s p}(6, \mathbb{R})$ & $\mathrm{R}$ \\
\hline$A_{1}+C_{7}$ & $\mathfrak{s l}(2, \mathbb{R}) \oplus \mathfrak{s p}(7, \mathbb{R})$ & $\mathrm{R}$ \\
\hline$A_{1}$ & $\mathfrak{s l}(2, \mathbb{R})$ & $\mathrm{S}$ \\
\hline$B_{2}$ & $\mathfrak{s o}(2,3)$ & $\mathrm{S}$ \\
\hline & $\mathfrak{s u}(2) \oplus \mathfrak{s o}(8)$ & \\
& $\mathfrak{s l}(2, \mathbb{R}) \oplus \mathfrak{s o}(8)$ & \\
& $\mathfrak{s l}(2, \mathbb{R}) \oplus \mathfrak{s o}(4,4)$ & $\mathrm{S}$ \\
$A_{1}+D_{4}$ & $\mathfrak{s l}(2, \mathbb{R}) \oplus \mathfrak{s o}(3,5)$ & \\
& $\mathfrak{s l}(2, \mathbb{R}) \oplus \mathfrak{s o}(1,7)$ & \\
\hline
\end{tabular}

Type $D_{8}$

Table 124: Maximal subalgebras of $\mathfrak{s o}(2,14)$.

\begin{tabular}{|c|c|c|}
\hline type & real subalgebras & \\
\hline$A_{7}$ & $\mathfrak{s} \mathfrak{u}(1,7) \oplus \mathfrak{u}(1)(2 \times)$ & $\overline{\mathrm{R}}$ \\
\hline \multirow{3}{*}{$A_{1}+A_{1}+D_{6}$} & $\mathfrak{s l}(2, \mathbb{R}) \oplus \mathfrak{s l}(2, \mathbb{R}) \oplus \mathfrak{s o}(12)$ & \multirow{3}{*}{$\mathrm{R}$} \\
\hline & $\mathfrak{s l}(2, \mathbb{C}) \oplus \mathfrak{s o}(11,1)$ & \\
\hline & $\mathfrak{s u}(2) \oplus \mathfrak{s u}(2) \oplus \mathfrak{s o}(2,10)$ & \\
\hline \multirow{3}{*}{$A_{3}+D_{5}$} & $\mathfrak{s u}(2,2) \oplus \mathfrak{s o}(10)$ & \multirow{3}{*}{$\mathrm{R}$} \\
\hline & $\mathfrak{s l}(2, \mathbb{H}) \oplus \mathfrak{s o}(9,1)$ & \\
\hline & $\mathfrak{s u}(4) \oplus \mathfrak{s o}(2,8)$ & \\
\hline \multirow{2}{*}{$D_{4}+D_{4}$} & $\mathfrak{s o}(8) \oplus \mathfrak{s o}^{*}(8)$ & \multirow{2}{*}{$\mathrm{R}$} \\
\hline & $\mathfrak{s o}(1,7) \oplus \mathfrak{s o}(1,7)$ & \\
\hline \multirow{3}{*}{$D_{7}+T_{1}$} & $\mathfrak{s o}(13,1) \oplus \mathfrak{s o}(1,1)$ & \multirow{3}{*}{$\mathrm{R}$} \\
\hline & $\mathfrak{s o}(2,12) \oplus \mathfrak{u}(1)$ & \\
\hline & $\mathfrak{s o}(14) \oplus \mathfrak{u}(1)$ & \\
\hline \multirow{3}{*}{$B_{3}+B_{4}$} & $\mathfrak{s o}(7) \oplus \mathfrak{s o}(2,7)$ & \multirow{3}{*}{$\mathrm{S}$} \\
\hline & $\mathfrak{s o}(2,5) \oplus \mathfrak{s o}(9)$ & \\
\hline & $\mathfrak{s o}(6,1) \oplus \mathfrak{s o}(8,1)$ & \\
\hline \multirow{3}{*}{$B_{2}+B_{5}$} & $\mathfrak{s o}(5) \oplus \mathfrak{s o}(2,9)$ & \multirow{3}{*}{$\mathrm{S}$} \\
\hline & $\mathfrak{s o}(2,3) \oplus \mathfrak{s o}(11)$ & \\
\hline & $\mathfrak{s o}(4,1) \oplus \mathfrak{s o}(10,1)$ & \\
\hline \multirow{3}{*}{$A_{1}+B_{6}$} & $\mathfrak{s u}(2) \oplus \mathfrak{s o}(2,11)$ & \multirow{3}{*}{$\mathrm{S}$} \\
\hline & $\mathfrak{s l}(2, \mathbb{R}) \oplus \mathfrak{s o}(13)$ & \\
\hline & $\mathfrak{s l}(2, \mathbb{R}) \oplus \mathfrak{s o}(12,1)$ & \\
\hline \multirow{2}{*}{$B_{7}$} & $\mathfrak{s o}(2,13)$ & \multirow{2}{*}{$\mathrm{S}$} \\
\hline & $\mathfrak{s o}(14,1)$ & \\
\hline
\end{tabular}

Table 125: Maximal subalgebras of $\mathfrak{s o}(4,12)$.

\begin{tabular}{|l|c|c|}
\hline type & real subalgebras & \\
\hline$A_{7}$ & $\mathfrak{s u}(2,6) \oplus \mathfrak{u}(1)(4 \times)$ & $\mathrm{R}$ \\
\hline
\end{tabular}




\begin{tabular}{|c|c|c|}
\hline \multicolumn{3}{|c|}{ subalgebras of $\mathfrak{s o}(4,12)$} \\
\hline \multirow{5}{*}{$A_{1}+A_{1}+D_{6}$} & $\mathfrak{s l}(2, \mathbb{R}) \oplus \mathfrak{s l}(2, \mathbb{R}) \oplus \mathfrak{s o}(2,10)$ & \multirow{5}{*}{$\mathrm{R}$} \\
\hline & $\mathfrak{s l}(2, \mathbb{C}) \oplus \mathfrak{s o}(3,9)$ & \\
\hline & $\mathfrak{s u}(2) \oplus \mathfrak{s u}(2) \oplus \mathfrak{s o}(4,8)$ & \\
\hline & $\mathfrak{s l}(2, \mathbb{C}) \oplus \mathfrak{s o}(11,1)$ & \\
\hline & $\mathfrak{s u}(2) \oplus \mathfrak{s u}(2) \oplus \mathfrak{s o}(12)$ & \\
\hline \multirow{5}{*}{$A_{3}+D_{5}$} & $\mathfrak{s l}(4, \mathbb{R}) \oplus \mathfrak{s o}(9,1)$ & \multirow{5}{*}{$\mathrm{R}$} \\
\hline & $\mathfrak{s u}(4) \oplus \mathfrak{s o}(4,6)$ & \\
\hline & $\mathfrak{s u}(2,2) \oplus \mathfrak{s o}(2,8)$ & \\
\hline & $\mathfrak{s l}(2, \mathbb{H}) \oplus \mathfrak{s o}(3,7)$ & \\
\hline & $\mathfrak{s u}(2,2) \oplus \mathfrak{s o}(10)$ & \\
\hline \multirow{3}{*}{$D_{4}+D_{4}$} & $\mathfrak{s o}(8) \oplus \mathfrak{s o}(4,4)$ & \multirow{3}{*}{$\mathrm{R}$} \\
\hline & $\mathfrak{s o}(3,5) \oplus \mathfrak{s o}(1,7)$ & \\
\hline & $\mathfrak{s o}^{*}(8) \oplus \mathfrak{s o}^{*}(8)$ & \\
\hline \multirow{3}{*}{$D_{7}+T_{1}$} & $\mathfrak{s o}(3,11) \oplus \mathfrak{s o}(1,1)$ & \multirow{3}{*}{$\mathrm{R}$} \\
\hline & $\mathfrak{s o}(4,10) \oplus \mathfrak{u}(1)$ & \\
\hline & $\mathfrak{s o}(2,12) \oplus \mathfrak{u}(1)$ & \\
\hline$A_{1}+C_{4}$ & $\mathfrak{s u}(2) \oplus \mathfrak{s p}(1,3)$ & $\mathrm{S}$ \\
\hline \multirow{5}{*}{$B_{3}+B_{4}$} & $\mathfrak{s o}(7) \oplus \mathfrak{s o}(4,5)$ & \multirow{5}{*}{$\mathrm{S}$} \\
\hline & $\mathfrak{s o}(2,5) \oplus \mathfrak{s o}(2,7)$ & \\
\hline & $\mathfrak{s o}(4,3) \oplus \mathfrak{s o}(9)$ & \\
\hline & $\mathfrak{s o}(4,3) \oplus \mathfrak{s o}(8,1)$ & \\
\hline & $\mathfrak{s o}(6,1) \oplus \mathfrak{s o}(6,3)$ & \\
\hline \multirow{5}{*}{$B_{2}+B_{5}$} & $\mathfrak{s o}(5) \oplus \mathfrak{s o}(4,7)$ & \multirow{5}{*}{$\mathrm{S}$} \\
\hline & $\mathfrak{s o}(2,3) \oplus \mathfrak{s o}(2,9)$ & \\
\hline & $\mathfrak{s o}(2,3) \oplus \mathfrak{s o}(10,1)$ & \\
\hline & $\mathfrak{s o}(4,1) \oplus \mathfrak{s o}(11)$ & \\
\hline & $\mathfrak{s o}(4,1) \oplus \mathfrak{s o}(8,3)$ & \\
\hline \multirow{4}{*}{$A_{1}+B_{6}$} & $\mathfrak{s u}(2) \oplus \mathfrak{s o}(4,9)$ & \multirow{4}{*}{$\mathrm{S}$} \\
\hline & $\mathfrak{s u}(2) \oplus \mathfrak{s o}(12,1)$ & \\
\hline & $\mathfrak{s l}(2, \mathbb{R}) \oplus \mathfrak{s o}(2,11)$ & \\
\hline & $\mathfrak{s l}(2, \mathbb{R}) \oplus \mathfrak{s o}(10,3)$ & \\
\hline \multirow{2}{*}{$B_{7}$} & $\mathfrak{s o}(4,11)$ & \multirow{2}{*}{$\mathrm{S}$} \\
\hline & $\mathfrak{s o}(12,3)$ & \\
\hline
\end{tabular}

Table 126: Maximal subalgebras of $\mathfrak{s o}(6,10)$.

\begin{tabular}{|l|r|r|}
\hline type & \multicolumn{1}{|c|}{ real subalgebras } & \\
\hline$A_{7}$ & $\mathfrak{s u}(3,5) \oplus \mathfrak{u}(1)(2 \times)$ & $\mathrm{R}$ \\
\hline & $\mathfrak{s l}(2, \mathbb{R}) \oplus \mathfrak{s l}(2, \mathbb{R}) \oplus \mathfrak{s o}(4,8)$ & \\
& $\mathfrak{s l}(2, \mathbb{C}) \oplus \mathfrak{s o}(5,7)$ & \\
$A_{1}+A_{1}+D_{6}$ & $\mathfrak{s u}(2) \oplus \mathfrak{s u}(2) \oplus \mathfrak{s o}(6,6)$ & $\mathrm{R}$ \\
& $\mathfrak{s l}(2, \mathbb{C}) \oplus \mathfrak{s o}(3,9)$ & \\
& $\mathfrak{s u}(2) \oplus \mathfrak{s u}(2) \oplus \mathfrak{s o}(2,10)$ & \\
\hline
\end{tabular}




\begin{tabular}{|c|c|c|}
\hline \multicolumn{3}{|c|}{ subalgebras of $\mathfrak{s o}(6,10)$} \\
\hline \multirow{7}{*}{$A_{3}+D_{5}$} & $\mathfrak{s l}(4, \mathbb{R}) \oplus \mathfrak{s o}(3,7)$ & \multirow{7}{*}{$\mathrm{R}$} \\
\hline & $\mathfrak{s u}(2,2) \oplus \mathfrak{s o}(4,6)$ & \\
\hline & $\mathfrak{s l}(2, \mathbb{H}) \oplus \mathfrak{s o}(5,5)$ & \\
\hline & $\mathfrak{s u}(2,2) \oplus \mathfrak{s o}(2,8)$ & \\
\hline & $\mathfrak{s u}(4) \oplus \mathfrak{s o}(4,6)$ & \\
\hline & $\mathfrak{s l}(2, \mathbb{H}) \oplus \mathfrak{s o}(9,1)$ & \\
\hline & $\mathfrak{s u}(4) \oplus \mathfrak{s o}(10)$ & \\
\hline \multirow{4}{*}{$D_{4}+D_{4}$} & $\mathfrak{s o}^{*}(8) \oplus \mathfrak{s o}(4,4)$ & \multirow{4}{*}{$\mathrm{R}$} \\
\hline & $\mathfrak{s o}(3,5) \oplus \mathfrak{s o}(3,5)$ & \\
\hline & $\mathfrak{s o}(3,5) \oplus \mathfrak{s o}(1,7)$ & \\
\hline & $\mathfrak{s o}(8) \oplus \mathfrak{s o}^{*}(8)$ & \\
\hline \multirow{3}{*}{$D_{7}+T_{1}$} & $\mathfrak{s o}(5,9) \oplus \mathfrak{s o}(1,1)$ & \multirow{3}{*}{$\mathrm{R}$} \\
\hline & $\mathfrak{s o}(6,8) \oplus \mathfrak{u}(1)$ & \\
\hline & $\mathfrak{s o}(4,10) \oplus \mathfrak{u}(1)$ & \\
\hline \multirow{7}{*}{$B_{3}+B_{4}$} & $\mathfrak{s o}(7) \oplus \mathfrak{s o}(6,3)$ & \multirow{7}{*}{$\mathrm{S}$} \\
\hline & $\mathfrak{s o}(2,5) \oplus \mathfrak{s o}(4,5)$ & \\
\hline & $\mathfrak{s o}(2,5) \oplus \mathfrak{s o}(8,1)$ & \\
\hline & $\mathfrak{s o}(4,3) \oplus \mathfrak{s o}(2,7)$ & \\
\hline & $\mathfrak{s o}(4,3) \oplus \mathfrak{s o}(6,3)$ & \\
\hline & $\mathfrak{s o}(6,1) \oplus \mathfrak{s o}(9)$ & \\
\hline & $\mathfrak{s o}(6,1) \oplus \mathfrak{s o}(4,5)$ & \\
\hline \multirow{6}{*}{$B_{2}+B_{5}$} & $\mathfrak{s o}(5) \oplus \mathfrak{s o}(6,5)$ & \multirow{6}{*}{$\mathrm{S}$} \\
\hline & $\mathfrak{s o}(5) \oplus \mathfrak{s o}(10,1)$ & \\
\hline & $\mathfrak{s o}(2,3) \oplus \mathfrak{s o}(4,7)$ & \\
\hline & $\mathfrak{s o}(2,3) \oplus \mathfrak{s o}(8,3)$ & \\
\hline & $\mathfrak{s o}(4,1) \oplus \mathfrak{s o}(2,9)$ & \\
\hline & $\mathfrak{s o}(4,1) \oplus \mathfrak{s o}(6,5)$ & \\
\hline \multirow{4}{*}{$A_{1}+B_{6}$} & $\mathfrak{s u}(2) \oplus \mathfrak{s o}(6,7)$ & \multirow{4}{*}{$\mathrm{S}$} \\
\hline & $\mathfrak{s u}(2) \oplus \mathfrak{s o}(10,3)$ & \\
\hline & $\mathfrak{s l}(2, \mathbb{R}) \oplus \mathfrak{s o}(4,9)$ & \\
\hline & $\mathfrak{s l}(2, \mathbb{R}) \oplus \mathfrak{s} \mathfrak{o}(8,5)$ & \\
\hline \multirow{2}{*}{$B_{7}$} & $\mathfrak{s o}(6,9)$ & \multirow{2}{*}{$\mathrm{S}$} \\
\hline & $\mathfrak{s o}(10,5)$ & \\
\hline
\end{tabular}

Table 127: Maximal subalgebras of $\mathfrak{s o}(8,8)$.

\begin{tabular}{|l|r|l|}
\hline type & real subalgebras & \\
\hline$A_{7}$ & $\mathfrak{s l}(8, \mathbb{R}) \oplus \mathfrak{s o}(1,1)(2 \times)$ & $\mathrm{R}$ \\
& $\mathfrak{s u}(4,4) \oplus \mathfrak{u}(1)(4 \times)$ & \\
\hline \multirow{3}{*}{$A_{1}+A_{1}+D_{6}$} & $\mathfrak{s l}(2, \mathbb{R}) \oplus \mathfrak{s l}(2, \mathbb{R}) \oplus \mathfrak{s o}(6,6)$ & \\
& $\mathfrak{s l}(2, \mathbb{C}) \oplus \mathfrak{s o}(5,7)(2 \times)$ & $\mathrm{R}$ \\
& $\mathfrak{s u}(2) \oplus \mathfrak{s u}(2) \oplus \mathfrak{s o}(4,8)(2 \times)$ & \\
\hline
\end{tabular}




\begin{tabular}{|c|c|c|}
\hline \multicolumn{3}{|c|}{ subalgebras of $\mathfrak{s o}(8,8)$} \\
\hline \multirow{4}{*}{$A_{3}+D_{5}$} & $\mathfrak{s l}(4, \mathbb{R}) \oplus \mathfrak{s o}(5,5)$ & \\
\hline & $\mathfrak{s u}(2,2) \oplus \mathfrak{s o}(4,6)(2 \times)$ & \\
\hline & $\mathfrak{s l}(2, \mathbb{H}) \oplus \mathfrak{s o}(3,7)(2 \times)$ & $\kappa$ \\
\hline & $\mathfrak{s u}(4) \oplus \mathfrak{s o}(2,8)(2 \times)$ & \\
\hline \multirow{6}{*}{$D_{4}+D_{4}$} & $\mathfrak{s o}(4,4) \oplus \mathfrak{s o}(4,4)$ & \\
\hline & $\mathfrak{s o}(3,5) \oplus \mathfrak{s o}(3,5)$ & \\
\hline & $\mathfrak{s o}(8, \mathbb{C})(2 \times)$ & \\
\hline & $\mathfrak{s o}^{*}(8) \oplus \mathfrak{s o}^{*}(8)$ & $\mathrm{R}$ \\
\hline & $\mathfrak{s o}(1,7) \oplus \mathfrak{s o}(1,7)$ & \\
\hline & $\mathfrak{s o}(8) \oplus \mathfrak{s o}(8)$ & \\
\hline \multirow{2}{*}{$D_{7}+T_{1}$} & $\mathfrak{s o}(7,7) \oplus \mathfrak{s o}(1,1)$ & 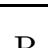 \\
\hline & $\mathfrak{s o}(6,8) \oplus \mathfrak{u}(1)(2 \times)$ & $\mathrm{R}$ \\
\hline \multirow{3}{*}{$B_{2}+B_{2}$} & $\mathfrak{s o}(5) \oplus \mathfrak{s o}(4,1)$ & \\
\hline & $\mathfrak{s o}(2,3) \oplus \mathfrak{s o}(2,3)$ & $\mathrm{S}$ \\
\hline & $\mathfrak{s o}(4,1) \oplus \mathfrak{s o}(4,1)$ & \\
\hline \multirow{2}{*}{$B_{4}$} & $\mathfrak{s o}(4,5)$ & \\
\hline & $\mathfrak{s o}(8,1)$ & $S$ \\
\hline \multirow{2}{*}{$A_{1}+C_{4}$} & $\mathfrak{s u}(2) \oplus \mathfrak{s p}(2,2)$ & $C$ \\
\hline & $\mathfrak{s l}(2, \mathbb{R}) \oplus \mathfrak{s p}(8, \mathbb{R})$ & $\mathrm{S}$ \\
\hline \multirow{4}{*}{$B_{3}+B_{4}$} & $\mathfrak{s o}(7) \oplus \mathfrak{s o}(8,1)$ & \\
\hline & $\mathfrak{s o}(2,5) \oplus \mathfrak{s o}(6,3)$ & $\mathrm{C}$ \\
\hline & $\mathfrak{s o}(4,3) \oplus \mathfrak{s o}(4,5)$ & $S$ \\
\hline & $\mathfrak{s o}(6,1) \oplus \mathfrak{s o}(2,7)$ & \\
\hline \multirow{3}{*}{$B_{2}+B_{5}$} & $\mathfrak{s o}(5) \oplus \mathfrak{s o}(8,3)$ & \\
\hline & $\mathfrak{s o}(2,3) \oplus \mathfrak{s o}(6,5)$ & $\mathrm{S}$ \\
\hline & $\mathfrak{s o}(4,1) \oplus \mathfrak{s o}(4,7)$ & \\
\hline \multirow{2}{*}{$A_{1}+B_{6}$} & $\mathfrak{s u}(2) \oplus \mathfrak{s o}(8,5)$ & \\
\hline & $\mathfrak{s l}(2, \mathbb{R}) \oplus \mathfrak{s o}(6,7)$ & $S$ \\
\hline$B_{7}$ & $\overline{\mathfrak{s o}(8,7)}$ & $\mathrm{S}$ \\
\hline
\end{tabular}

Table 128: Maximal subalgebras of $\mathfrak{s o}^{*}(16)$.

\begin{tabular}{|l|c|l|}
\hline type & real subalgebras & \\
\hline & $\mathfrak{s u}(3,5) \oplus \mathfrak{u}(1)$ & \\
& $\mathfrak{s u}(2,6) \oplus \mathfrak{u}(1)$ & \\
$A_{7}$ & $\mathfrak{s u}(1,7) \oplus \mathfrak{u}(1)$ & \\
& $\mathfrak{s l}(4, \mathbb{H}) \oplus \mathfrak{s o}(1,1)$ & $\mathrm{R}$ \\
& $\mathfrak{s u}(4,4) \oplus \mathfrak{u}(1)$ & \\
& $\mathfrak{s u}(8) \oplus \mathfrak{u}(1)$ & \\
\hline$A_{1}+A_{1}+D_{6}$ & $\mathfrak{s u}(2) \oplus \mathfrak{s l}(2, \mathbb{R}) \oplus \mathfrak{s o}(12)$ & $\mathrm{R}$ \\
\hline$A_{3}+D_{5}$ & $\mathfrak{s u}(1,3) \oplus \mathfrak{s 0 ^ { * }}(10)$ & $\mathrm{R}$ \\
\hline$D_{4}+D_{4}$ & $\mathfrak{s o}^{*}(8) \oplus \mathfrak{s o}(8)$ & $\mathrm{R}$ \\
\hline$D_{7}+T_{1}$ & $\mathfrak{s o}^{*}(8, \mathbb{C})$ & \\
\hline
\end{tabular}




\begin{tabular}{|l|c|l|}
\hline \multicolumn{2}{|l|}{ subalgebras of $\mathfrak{s o}^{*}(16)$} \\
\hline$B_{2}+B_{2}$ & $\begin{array}{r}\mathfrak{s o}(5) \oplus \mathfrak{s o}(2,3) \\
\mathfrak{s o}(2,3) \oplus \mathfrak{s o}(4,1)\end{array}$ & $\mathrm{S}$ \\
\hline$B_{4}$ & $\mathfrak{s o}(2,7)$ & $\mathrm{S}$ \\
\hline \multirow{5}{*}{$A_{1}+C_{4}$} & $\mathfrak{s o}(6,3)$ & \\
& $\mathfrak{s u}(2) \oplus \mathfrak{s p}(4, \mathbb{R})$ & \\
& $\mathfrak{s l}(2, \mathbb{R}) \oplus \mathfrak{s p}(4)$ & $\mathrm{S}$ \\
& $\mathfrak{s l}(2, \mathbb{R}) \oplus \mathfrak{s p}(1,3)$ & \\
& $\mathfrak{s l}(2, \mathbb{R}) \oplus \mathfrak{s p}(2,2)$ & \\
\hline
\end{tabular}

Table 129: Maximal subalgebras of $\mathfrak{s o}(15,1)$.

\begin{tabular}{|c|c|c|}
\hline type & real subalgebras & \\
\hline \multirow{2}{*}{$A_{1}+A_{1}+D_{6}$} & $\mathfrak{s l}(2, \mathbb{C}) \oplus \mathfrak{s o}(12)$ & \multirow{2}{*}{$\mathrm{R}$} \\
\hline & $\mathfrak{s u}(2) \oplus \mathfrak{s u}(2) \oplus \mathfrak{s o}(11,1)$ & \\
\hline \multirow{2}{*}{$A_{3}+D_{5}$} & $\mathfrak{s l}(2, \mathbb{H}) \oplus \mathfrak{s o}(10)$ & \multirow{2}{*}{$\mathrm{R}$} \\
\hline & $\mathfrak{s u}(4) \oplus \mathfrak{s o}(9,1)$ & \\
\hline$D_{4}+D_{4}$ & $\mathfrak{s o}(8) \oplus \mathfrak{s o}(1,7)$ & $\mathrm{R}$ \\
\hline \multirow{2}{*}{$D_{7}+T_{1}$} & $\mathfrak{s o}(13,1) \oplus \mathfrak{u}(1)$ & \multirow{2}{*}{$\mathrm{R}$} \\
\hline & $\mathfrak{s o}(14) \oplus \mathfrak{s o}(1,1)$ & \\
\hline \multirow{2}{*}{$B_{3}+B_{4}$} & $\mathfrak{s o}(7) \oplus \mathfrak{s o}(8,1)$ & \multirow{2}{*}{$\mathrm{S}$} \\
\hline & $\mathfrak{s o}(6,1) \oplus \mathfrak{s o}(9)$ & \\
\hline \multirow{2}{*}{$B_{2}+B_{5}$} & $\mathfrak{s o}(5) \oplus \mathfrak{s o}(10,1)$ & \multirow{2}{*}{$\mathrm{S}$} \\
\hline & $\mathfrak{s o}(4,1) \oplus \mathfrak{s o}(11)$ & \\
\hline \multirow{2}{*}{$A_{1}+B_{6}$} & $\mathfrak{s u}(2) \oplus \mathfrak{s o}(12,1)$ & \multirow[b]{2}{*}{$\mathrm{S}$} \\
\hline & $\mathfrak{s l}(2, \mathbb{R}) \oplus \mathfrak{s o}(13)$ & \\
\hline \multirow{2}{*}{$B_{7}$} & $\mathfrak{s o}(15)$ & \multirow{2}{*}{ S } \\
\hline & $\mathfrak{s o}(14,1)$ & \\
\hline
\end{tabular}

Table 130: Maximal subalgebras of $\mathfrak{s o}(3,13)$.

\begin{tabular}{|l|r|l|}
\hline type & real subalgebras & \\
\hline & $\mathfrak{s l}(2, \mathbb{R}) \oplus \mathfrak{s l}(2, \mathbb{R}) \oplus \mathfrak{s o}(11,1)$ & \\
$A_{1}+A_{1}+D_{6}$ & $\mathfrak{s l}(2, \mathbb{C}) \oplus \mathfrak{s o}(2,10)$ & \\
& $\mathfrak{s u}(2) \oplus \mathfrak{s u}(2) \oplus \mathfrak{s o}(3,9)$ & $\mathrm{R}$ \\
& $\mathfrak{s l}(2, \mathbb{C}) \oplus \mathfrak{s o}(12)$ & \\
\hline & $\mathfrak{s l}(4, \mathbb{R}) \oplus \mathfrak{s o}(10)$ & \\
$A_{3}+D_{5}$ & $\mathfrak{s u}(2,2) \oplus \mathfrak{s o}(9,1)$ & $\mathrm{R}$ \\
& $\mathfrak{s l}(2, \mathbb{H}) \oplus \mathfrak{s o}(2,8)$ & \\
& $\mathfrak{s u}(4) \oplus \mathfrak{s o}(3,7)$ & \\
\hline \multirow{2}{*}{$D_{4}+D_{4}$} & $\mathfrak{s o}(8) \oplus \mathfrak{s o}(3,5)$ & $\mathrm{R}$ \\
\hline & $\mathfrak{s o}(8) \oplus \mathfrak{s o}(1,7)$ & \\
$D_{7}+T_{1}$ & $\mathfrak{s o}(2,12) \oplus \mathfrak{s o}(1,1)$ & $\mathrm{R}$ \\
& $\mathfrak{s o}(3,11) \oplus \mathfrak{u}(1)$ & \\
\hline
\end{tabular}




\begin{tabular}{|c|c|c|}
\hline \multicolumn{3}{|c|}{ subalgebras of $\mathfrak{s o}(3,13)$} \\
\hline \multirow{4}{*}{$B_{3}+B_{4}$} & $\mathfrak{s o}(7) \oplus \mathfrak{s o}(6,3)$ & \\
\hline & $\mathfrak{s o}(2,5) \oplus \mathfrak{s o}(8,1)$ & \\
\hline & $\mathfrak{s o}(4,3) \oplus \mathfrak{s o}(9)$ & $S$ \\
\hline & $\mathfrak{s o}(6,1) \oplus \mathfrak{s o}(2,7)$ & \\
\hline \multirow{4}{*}{$B_{2}+B_{5}$} & $\mathfrak{s o}(5) \oplus \mathfrak{s o}(8,3)$ & \\
\hline & $\mathfrak{s o}(2,3) \oplus \mathfrak{s o}(11)$ & \\
\hline & $\mathfrak{s o}(2,3) \oplus \mathfrak{s o}(10,1)$ & $S$ \\
\hline & $\mathfrak{s o}(4,1) \oplus \mathfrak{s o}(2,9)$ & \\
\hline \multirow{4}{*}{$A_{1}+B_{6}$} & $\mathfrak{s u}(2) \oplus \mathfrak{s o}(13)$ & \\
\hline & $\mathfrak{s u}(2) \oplus \mathfrak{s o}(10,3)$ & \\
\hline & $\mathfrak{s l}(2, \mathbb{R}) \oplus \mathfrak{s o}(2,11)$ & $\mathrm{S}$ \\
\hline & $\mathfrak{s l}(2, \mathbb{R}) \oplus \mathfrak{s o}(12,1)$ & \\
\hline \multirow{2}{*}{$B_{7}$} & $\mathfrak{s o}(2,13)$ & $\mathrm{C}$ \\
\hline & $\mathfrak{s o}(12,3)$ & $S$ \\
\hline
\end{tabular}

Table 131: Maximal subalgebras of $\mathfrak{s o}(5,11)$.

\begin{tabular}{|c|c|c|}
\hline type & real subalgebras & \\
\hline \multirow{5}{*}{$A_{1}+A_{1}+D_{6}$} & $\mathfrak{s l}(2, \mathbb{R}) \oplus \mathfrak{s l}(2, \mathbb{R}) \oplus \mathfrak{s o}(3,9)$ & \multirow{5}{*}{$\mathrm{R}$} \\
\hline & $\mathfrak{s l}(2, \mathbb{C}) \oplus \mathfrak{s o}(4,8)$ & \\
\hline & $\mathfrak{s u}(2) \oplus \mathfrak{s u}(2) \oplus \mathfrak{s o}(5,7)$ & \\
\hline & $\mathfrak{s l}(2, \mathbb{C}) \oplus \mathfrak{s o}(2,10)$ & \\
\hline & $\mathfrak{s u}(2) \oplus \mathfrak{s u}(2) \oplus \mathfrak{s o}(11,1)$ & \\
\hline \multirow{6}{*}{$A_{3}+D_{5}$} & $\mathfrak{s l}(4, \mathbb{R}) \oplus \mathfrak{s o}(2,8)$ & \multirow{6}{*}{$\mathrm{R}$} \\
\hline & $\mathfrak{s u}(2,2) \oplus \mathfrak{s o}(3,7)$ & \\
\hline & $\mathfrak{s l}(2, \mathbb{H}) \oplus \mathfrak{s o}(4,6)$ & \\
\hline & $\mathfrak{s u}(4) \oplus \mathfrak{s o}(5,5)$ & \\
\hline & $\mathfrak{s u}(2,2) \oplus \mathfrak{s o}(9,1)$ & \\
\hline & $\mathfrak{s l}(2, \mathbb{H}) \oplus \mathfrak{s o}(10)$ & \\
\hline \multirow{3}{*}{$D_{4}+D_{4}$} & $\mathfrak{s o}(4,4) \oplus \mathfrak{s o}(1,7)$ & \multirow{3}{*}{$\mathrm{R}$} \\
\hline & $\mathfrak{s o}^{*}(8) \oplus \mathfrak{s o}(3,5)$ & \\
\hline & $\mathfrak{s o}(8) \oplus \mathfrak{s o}(3,5)$ & \\
\hline \multirow{3}{*}{$D_{7}+T_{1}$} & $\mathfrak{s o}(4,10) \oplus \mathfrak{s o}(1,1)$ & \multirow{3}{*}{$\mathrm{R}$} \\
\hline & $\mathfrak{s o}(5,9) \oplus \mathfrak{u}(1)$ & \\
\hline & $\mathfrak{s o}(3,11) \oplus \mathfrak{u}(1)$ & \\
\hline \multirow{6}{*}{$B_{3}+B_{4}$} & $\mathfrak{s o}(7) \oplus \mathfrak{s o}(4,5)$ & \multirow{6}{*}{$\mathrm{S}$} \\
\hline & $\mathfrak{s o}(2,5) \oplus \mathfrak{s o}(9)$ & \\
\hline & $\mathfrak{s o}(2,5) \oplus \mathfrak{s o}(6,3)$ & \\
\hline & $\mathfrak{s o}(4,3) \oplus \mathfrak{s o}(2,7)$ & \\
\hline & $\mathfrak{s o}(4,3) \oplus \mathfrak{s o}(8,1)$ & \\
\hline & $\mathfrak{s o}(6,1) \oplus \mathfrak{s o}(4,5)$ & \\
\hline
\end{tabular}




\begin{tabular}{|c|c|c|}
\hline \multicolumn{3}{|c|}{ subalgebras of $\mathfrak{s o}(5,11)$} \\
\hline \multirow{6}{*}{$B_{2}+B_{5}$} & $\mathfrak{s o}(5) \oplus \mathfrak{s o}(11)$ & \multirow{6}{*}{ S } \\
\hline & $\mathfrak{s o}(5) \oplus \mathfrak{s o}(6,5)$ & \\
\hline & $\mathfrak{s o}(2,3) \oplus \mathfrak{s o}(2,9)$ & \\
\hline & $\mathfrak{s o}(2,3) \oplus \mathfrak{s o}(8,3)$ & \\
\hline & $\mathfrak{s o}(4,1) \oplus \mathfrak{s o}(4,7)$ & \\
\hline & $\mathfrak{s o}(4,1) \oplus \mathfrak{s o}(10,1)$ & \\
\hline \multirow{4}{*}{$A_{1}+B_{6}$} & $\mathfrak{s u}(2) \oplus \mathfrak{s o}(2,11)$ & \multirow{4}{*}{$\mathrm{S}$} \\
\hline & $\mathfrak{s u}(2) \oplus \mathfrak{s o}(8,5)$ & \\
\hline & $\mathfrak{s l}(2, \mathbb{R}) \oplus \mathfrak{s o}(4,9)$ & \\
\hline & $\mathfrak{s l}(2, \mathbb{R}) \oplus \mathfrak{s o}(10,3)$ & \\
\hline \multirow{2}{*}{$B_{7}$} & $\mathfrak{s o}(4,11)$ & \multirow{2}{*}{$\mathrm{S}$} \\
\hline & $\mathfrak{s o}(10,5)$ & \\
\hline
\end{tabular}

Table 132: Maximal subalgebras of $\mathfrak{s o}(7,9)$.

\begin{tabular}{|c|c|c|}
\hline type & real subalgebras & \\
\hline \multirow{5}{*}{$A_{1}+A_{1}+D_{6}$} & $\mathfrak{s l}(2, \mathbb{R}) \oplus \mathfrak{s l}(2, \mathbb{R}) \oplus \mathfrak{s o}(5,7)$ & \multirow{5}{*}{$\mathrm{R}$} \\
\hline & $\mathfrak{s l}(2, \mathbb{C}) \oplus \mathfrak{s o}(6,6)$ & \\
\hline & $\mathfrak{s l}(2, \mathbb{C}) \oplus \mathfrak{s o}(4,8)$ & \\
\hline & $\mathfrak{s u}(2) \oplus \mathfrak{s u}(2) \oplus \mathfrak{s o}(5,7)$ & \\
\hline & $\mathfrak{s u}(2) \oplus \mathfrak{s u}(2) \oplus \mathfrak{s o}(3,9)$ & \\
\hline \multirow{7}{*}{$A_{3}+D_{5}$} & $\mathfrak{s l}(4, \mathbb{R}) \oplus \mathfrak{s o}(4,6)$ & \multirow{7}{*}{$\mathrm{R}$} \\
\hline & $\mathfrak{s u}(2,2) \oplus \mathfrak{s o}(5,5)$ & \\
\hline & $\mathfrak{s u}(2,2) \oplus \mathfrak{s o}(3,7)$ & \\
\hline & $\mathfrak{s l}(2, \mathbb{H}) \oplus \mathfrak{s o}(4,6)$ & \\
\hline & $\mathfrak{s l}(2, \mathbb{H}) \oplus \mathfrak{s o}(2,8)$ & \\
\hline & $\mathfrak{s u}(4) \oplus \mathfrak{s o}(3,7)$ & \\
\hline & $\mathfrak{s u}(4) \oplus \mathfrak{s o}(9,1)$ & \\
\hline \multirow{4}{*}{$D_{4}+D_{4}$} & $\mathfrak{s o}(4,4) \oplus \mathfrak{s o}(3,5)$ & \multirow{4}{*}{$\mathrm{R}$} \\
\hline & $\mathfrak{s o}^{*}(8) \oplus \mathfrak{s o}(3,5)$ & \\
\hline & $\mathfrak{s o}^{*}(8) \oplus \mathfrak{s o}(1,7)$ & \\
\hline & $\mathfrak{s o}(8) \oplus \mathfrak{s o}(1,7)$ & \\
\hline \multirow{3}{*}{$D_{7}+T_{1}$} & $\mathfrak{s o}(6,8) \oplus \mathfrak{s o}(1,1)$ & \multirow{3}{*}{$\mathrm{R}$} \\
\hline & $\mathfrak{s o}(7,7) \oplus \mathfrak{u}(1)$ & \\
\hline & $\mathfrak{s o}(5,9) \oplus \mathfrak{u}(1)$ & \\
\hline \multirow{8}{*}{$B_{3}+B_{4}$} & $\mathfrak{s o}(7) \oplus \mathfrak{s o}(9)$ & \multirow{8}{*}{$\mathrm{S}$} \\
\hline & $\mathfrak{s o}(7) \oplus \mathfrak{s o}(2,7)$ & \\
\hline & $\mathfrak{s o}(2,5) \oplus \mathfrak{s o}(2,7)$ & \\
\hline & $\mathfrak{s o}(2,5) \oplus \mathfrak{s o}(4,5)$ & \\
\hline & $\mathfrak{s o}(4,3) \oplus \mathfrak{s o}(4,5)$ & \\
\hline & $\mathfrak{s o}(4,3) \oplus \mathfrak{s o}(6,3)$ & \\
\hline & $\mathfrak{s o}(6,1) \oplus \mathfrak{s o}(6,3)$ & \\
\hline & $\mathfrak{s o}(6,1) \oplus \mathfrak{s o}(8,1)$ & \\
\hline
\end{tabular}




\begin{tabular}{|c|c|c|}
\hline \multicolumn{3}{|c|}{ subalgebras of $\mathfrak{s o}(7,9)$} \\
\hline \multirow{6}{*}{$B_{2}+B_{5}$} & $\mathfrak{s o}(5) \oplus \mathfrak{s o}(2,9)$ & \multirow{6}{*}{$\mathrm{S}$} \\
\hline & $\mathfrak{s o}(5) \oplus \mathfrak{s o}(4,7)$ & \\
\hline & $\mathfrak{s o}(2,3) \oplus \mathfrak{s} \mathfrak{o}(4,7)$ & \\
\hline & $\mathfrak{s o}(2,3) \oplus \mathfrak{s o}(6,5)$ & \\
\hline & $\mathfrak{s o}(4,1) \oplus \mathfrak{s o}(6,5)$ & \\
\hline & $\mathfrak{s o}(4,1) \oplus \mathfrak{s o}(8,3)$ & \\
\hline \multirow{4}{*}{$A_{1}+B_{6}$} & $\mathfrak{s u}(2) \oplus \mathfrak{s o}(4,9)$ & \multirow{4}{*}{$\mathrm{S}$} \\
\hline & $\mathfrak{s u}(2) \oplus \mathfrak{s o}(6,7)$ & \\
\hline & $\mathfrak{s l}(2, \mathbb{R}) \oplus \mathfrak{s o}(6,7)$ & \\
\hline & $\mathfrak{s l}(2, \mathbb{R}) \oplus \mathfrak{s o}(8,5)$ & \\
\hline \multirow{2}{*}{$B_{7}$} & $\mathfrak{s o}(6,9)$ & \multirow{2}{*}{$\mathrm{S}$} \\
\hline & $\mathfrak{s o}(8,7)$ & \\
\hline
\end{tabular}

Type $E_{8}$

Table 133: Maximal subalgebras of $E_{8}(8)$.

\begin{tabular}{|c|c|c|}
\hline type & real subalgebras & \\
\hline \multirow{4}{*}{$2 A_{4}$} & $\mathfrak{s u}(5) \oplus \mathfrak{s u}(1,4)$ & \multirow{4}{*}{$\mathrm{R}$} \\
\hline & $\mathfrak{s u}(1,4) \oplus \mathfrak{s u}(1,4)$ & \\
\hline & $\mathfrak{s u}(2,3) \oplus \mathfrak{s u}(2,3)$ & \\
\hline & $\mathfrak{s l}(5, \mathbb{R}) \oplus \mathfrak{s l}(5, \mathbb{R})$ & \\
\hline \multirow{3}{*}{$D_{8}$} & $\mathfrak{s o}(16)$ & \multirow{3}{*}{$\mathrm{R}$} \\
\hline & $\mathfrak{s o}(8,8)$ & \\
\hline & $\mathfrak{s o}^{*}(16)$ & \\
\hline \multirow{3}{*}{$A_{8}$} & $\mathfrak{s u}(1,8)$ & \multirow{3}{*}{$\mathrm{R}$} \\
\hline & $\mathfrak{s u}(4,5)$ & \\
\hline & $\mathfrak{s l}(9, \mathbb{R})$ & \\
\hline \multirow{3}{*}{$A_{2}+E_{6}$} & $\mathfrak{s l}(3, \mathbb{R}) \oplus E_{6(6)}$ & \multirow{3}{*}{$\mathrm{R}$} \\
\hline & $\mathfrak{s u}(3) \oplus E_{6(-14)}$ & \\
\hline & $\mathfrak{s u}(1,2) \oplus E_{6(2)}$ & \\
\hline \multirow{2}{*}{$A_{1}+E_{7}$} & $\mathfrak{s u}(2) \oplus E_{7(-5)}$ & \multirow{2}{*}{$\mathrm{R}$} \\
\hline & $\mathfrak{s l}(2, \mathbb{R}) \oplus E_{7(7)}$ & \\
\hline \multirow{2}{*}{$B_{2}$} & $\mathfrak{s o}(2,3)$ & \multirow{2}{*}{$\mathrm{S}$} \\
\hline & $\mathfrak{s o}(4,1)$ & \\
\hline \multirow{4}{*}{$A_{1}+A_{2}$} & $\mathfrak{s u}(2) \oplus \mathfrak{s u}(1,2)$ & \multirow{4}{*}{$\mathrm{S}$} \\
\hline & $\mathfrak{s l}(2, \mathbb{R}) \oplus \mathfrak{s} \mathfrak{u}(3)$ & \\
\hline & $\mathfrak{s l}(2, \mathbb{R}) \oplus \mathfrak{s u}(1,2)$ & \\
\hline & $\mathfrak{s l}(2, \mathbb{R}) \oplus \mathfrak{s l}(3, \mathbb{R})$ & \\
\hline$F_{4}+G_{2}$ & $\begin{array}{c}F_{4(-20)} \oplus G_{2}^{\mathrm{cpt}} \\
F_{4(4)} \oplus G_{2}(2)\end{array}$ & $\mathrm{S}$ \\
\hline$A_{1}^{520}$ & $\mathfrak{s l}(2, \mathbb{R})$ & $\mathrm{S}$ \\
\hline$A_{1}^{760}$ & $\mathfrak{s l}(2, \mathbb{R})$ & $\bar{S}$ \\
\hline$A_{1}^{1240}$ & $\overline{\mathfrak{s l}(2, \mathbb{R})}$ & $\mathrm{S}$ \\
\hline
\end{tabular}


Table 134: Maximal subalgebras of $E_{8}(-24)$.

\begin{tabular}{|l|c|l|}
\hline type & real subalgebras & \\
\hline $2 A_{4}$ & $\begin{array}{c}\mathfrak{s u}(1,4) \oplus \mathfrak{s u}(2,3) \\
\mathfrak{s u}(5) \oplus \mathfrak{s u}(2,3)\end{array}$ & $\mathrm{R}$ \\
\hline$D_{8}$ & $\mathfrak{s o}(4,12)$ & $\mathrm{R}$ \\
& $\mathfrak{s o}(16)$ & \\
\hline$A_{8}$ & $\mathfrak{s u}(3,6)$ & $\mathrm{R}$ \\
\hline & $\mathfrak{s u}(2,7)$ & \\
& $\mathfrak{s u}(3) \oplus E_{6(2)}$ & \\
$A_{2}+E_{6}$ & $\mathfrak{s u}(1,2) \oplus E_{6(-14)}$ & $\mathrm{R}$ \\
& $\mathfrak{s u}(1,2) \oplus E_{6}^{\mathrm{cpt}}$ & \\
& $\mathfrak{s l}(3, \mathbb{R}) \oplus E_{6(-26)}$ & \\
\hline & $\mathfrak{s u}(2) \oplus E_{7(-5)}$ & \\
$A_{1}+E_{7}$ & $\mathfrak{s u}(2) \oplus E_{7}^{\mathrm{cpt}}$ & $\mathrm{R}$ \\
& $\mathfrak{s l}(2, \mathbb{R}) \oplus E_{7(-25)}$ & \\
\hline$A_{1}+A_{2}$ & $\mathfrak{s u}(2) \oplus \mathfrak{s l}\left(3, \mathbb{R}^{\mathrm{c}}\right)$ & $\mathrm{S}$ \\
\hline & $F_{4(4)} \oplus G_{2}^{\mathrm{cpt}}$ & \\
$F_{4}+G_{2}$ & $F_{4}^{\mathrm{cpt}} \oplus G_{2(2)}$ & $\mathrm{S}$ \\
& $F_{4(-20)} \oplus G_{2(2)}$ & \\
\hline
\end{tabular}

\section{REFERENCES}

[AC09] Dmitri V. Alekseevsky and Vicente Cortés. Geometric construction of the r-map: from affine special real to special Kähler manifolds. Commun. Math. Phys., 291:579$590,2009$.

[AFMT08] L. Andrianopoli, S. Ferrara, A. Marrani, and M. Trigiante. Non-BPS Attractors in 5d and 6d Extended Supergravity. Nucl. Phys., B795:428-452, 2008.

[BDS49] A. Borel and J. De Siebenthal. Les sous-groupes fermés de rang maximum des groupes de Lie clos. Comment. Math. Helv., 23:200-221, 1949.

[BFKM08] Stefano Bellucci, Sergio Ferrara, Renata Kallosh, and Alessio Marrani. Extremal Black Hole and Flux Vacua Attractors. Lect. Notes Phys., 755:115-191, 2008.

[BMG88] Peter Breitenlohner, Dieter Maison, and Gary W. Gibbons. Four-Dimensional Black Holes from Kaluza-Klein Theories. Commun. Math. Phys., 120:295, 1988.

[Bro69] R. B. Brown. Groups of type e $e_{7}$. J. Reine Angew. Math., 236:79-102, 1969.

[BS03] C. H. Barton and A. Sudbery. Magic squares and matrix models of Lie algebras. Adv. Math, 180:596-647, 2003.

[CCM13] Sergio L. Cacciatori, Bianca Letizia Cerchiai, and Alessio Marrani. Magic Coset Decompositions. Adv. Theor. Math. Phys., 17(5):1077-1128, 2013.

[CCM15] Sergio L. Cacciatori, Bianca L. Cerchiai, and Alessio Marrani. Squaring the Magic. Adv. Theor. Math. Phys., 19:923-954, 2015.

[CFG89] S. Cecotti, S. Ferrara, and L. Girardello. Geometry of Type II Superstrings and the Moduli of Superconformal Field Theories. Int. J. Mod. Phys., A4:2475, 1989.

[CFMZ10] Bianca L. Cerchiai, Sergio Ferrara, Alessio Marrani, and Bruno Zumino. Charge Orbits of Extremal Black Holes in Five Dimensional Supergravity. Phys. Rev., D82:085010, 2010.

[CJ78] E. Cremmer and B. Julia. The N=8 Supergravity Theory. 1. The Lagrangian. Phys. Lett., 80B:48, 1978 .

[CJ79] E. Cremmer and B. Julia. The SO(8) Supergravity. Nucl. Phys., B159:141-212, 1979.

$\left[\mathrm{CLL}^{+} 98\right] \quad$ E. Cremmer, I. V. Lavrinenko, Hong Lu, C. N. Pope, K. S. Stelle, and Tuan A. Tran. Euclidean signature supergravities, dualities and instantons. Nucl. Phys., B534:40$82,1998$.

[CLO15] David A. Cox, John Little, and Donal O'Shea. Ideals, varieties, and algorithms. Undergraduate Texts in Mathematics. Springer, Cham, fourth edition, 2015. An introduction to computational algebraic geometry and commutative algebra. 
[CM93] David H. Collingwood and William M. McGovern. Nilpotent orbits in semisimple Lie algebras. Van Nostrand Reinhold Mathematics Series. Van Nostrand Reinhold Co., New York, 1993.

[DFdG15] Heiko Dietrich, Paolo Faccin, and Willem A. de Graaf. Regular subalgebras and nilpotent orbits of real graded Lie algebras. J. Algebra, 423:1044-1079, 2015.

[DFdG19] H. Dietrich, P. Faccin, and W. A. de Graaf. CoReLG, computation with real lie groups, Version 1.53. https://gap-packages.github.io/corelg/ Nov 2019. Refereed GAP package.

[DFG13] Heiko Dietrich, Paolo Faccin, and Willem A. de Graaf. Computing with real Lie algebras: real forms, Cartan decompositions, and Cartan subalgebras. J. Symbolic Comput., 56:27-45, 2013.

[dG11] W. A. de Graaf. Constructing semisimple subalgebras of semisimple Lie algebras. J. of Algebra, 325:416-430, 2011.

[DG19] Heiko Dietrich and Willem A. de Graaf. Computing the real Weyl group. preprint, arXiv:1907.01398 [math.RT], 2019.

[dGGT19] W. A. de Graaf and T. GAP Team. SLA, computing with simple lie algebras, Version 1.5.2. https://gap-packages.github.io/sla/, Mar 2019. Refereed GAP package.

[dWVVP93] B. de Wit, F. Vanderseypen, and Antoine Van Proeyen. Symmetry structure of special geometries. Nucl. Phys., B400:463-524, 1993.

[Dyn52a] E. B. Dynkin. Maximal subgroups of classical groups. Trudy Moskov. Mat. Obshch, 1:39-166, 1952. English translation in: Amer. Math. Soc. Transl. (2) vol. 6 (1957), $245-378$.

[Dyn52b] E. B. Dynkin. Semisimple subalgebras of semisimple Lie algebras. Mat. Sbornik N.S., 30(72):349-462 (3 plates), 1952. English translation in: Amer. Math. Soc. Transl. (6), (1957), 111-244.

[FdG15] Paolo Faccin and Willem A. de Graaf. Constructing semisimple subalgebras of real semisimple Lie algebras. In Lie algebras and related topics, volume 652 of Contemp. Math., pages 75-89. Amer. Math. Soc., Providence, RI, 2015.

[FMT12] Sergio Ferrara, Alessio Marrani, and Mario Trigiante. Super-Ehlers in any dimension. J. High Energy Phys., (11):068, front matter + 63, 2012.

[FMZ13] Sergio Ferrara, Alessio Marrani, and Bruno Zumino. Jordan Pairs, E6 and U-Duality in Five Dimensions. J. Phys., A46:065402, 2013.

[Fre63] H. Freudenthal. Lie groups in the foundations of geometry. Adv. Math, 1:145, 1963.

[GAP18] GAP - Groups, Algorithms, and Programming, Version 4.10.0. https://www.gap-system.org, Nov 2018.

[GG78] Morikuni Goto and Frank D. Grosshans. Semisimple Lie algebras. Marcel Dekker, Inc., New York-Basel, 1978. Lecture Notes in Pure and Applied Mathematics, Vol. 38 .

[Gil06] R. Gilmore. Lie Groups, Lie Algebras and some of their Applications. Dover Books on Mathematics. Dover, paperback version edition, 2006.

[GKN01] M. Gunaydin, K. Koepsell, and H. Nicolai. Conformal and quasiconformal realizations of exceptional Lie groups. Commun. Math. Phys., 221:57-76, 2001.

[GNPP08] Murat Gunaydin, Andrew Neitzke, Oleksandr Pavlyk, and Boris Pioline. Quasiconformal actions, quaternionic discrete series and twistors: $S U(2,1)$ and $G_{2}(2)$. Commun. Math. Phys., 283:169-226, 2008.

[GNPW06] Murat Gunaydin, Andrew Neitzke, Boris Pioline, and Andrew Waldron. BPS black holes, quantum attractor flows and automorphic forms. Phys. Rev., D73:084019, 2006.

[GNPW07] Murat Gunaydin, Andrew Neitzke, Boris Pioline, and Andrew Waldron. Quantum Attractor Flows. JHEP, 09:056, 2007.

[GP09] Murat Gunaydin and Oleksandr Pavlyk. Quasiconformal Realizations of E(6)(6), $\mathrm{E}(7)(7), \mathrm{E}(8)(8)$ and $\mathrm{SO}(\mathrm{n}+3, \mathrm{~m}+3), \mathrm{N}_{i}=4$ Supergravity and Spherical Vectors. Adv. Theor. Math. Phys., 13(6):1895-1940, 2009.

[GP10] Murat Gunaydin and Oleksandr Pavlyk. Spectrum Generating Conformal and Quasiconformal U-Duality Groups, Supergravity and Spherical Vectors. JHEP, 04:070, 2010 .

[Gra72] A. Gray. Riemannian manifolds with geodesic symmetries of order 3. J. Diff. Geom., 7:343-369, 1972.

[Gra17] Willem A. de Graaf. Computation with linear algebraic groups. Monographs and Research Notes in Mathematics. CRC Press, Boca Raton, FL, 2017.

[GSS11] M. Gunaydin, H. Samtleben, and E. Sezgin. On the Magical Supergravities in Six Dimensions. Nucl. Phys., B848:62-89, 2011. 
[GST83] M. Gunaydin, G. Sierra, and P. K. Townsend. Exceptional Supergravity Theories and the Magic Square. Phys. Lett., 133B:72-76, 1983.

[Gun75] Murat Gunaydin. Exceptional Realizations of Lorentz Group: Supersymmetries and Leptons. Nuovo Cim., A29:467, 1975.

[Gun93] Murat Gunaydin. Generalized conformal and superconformal group actions and Jordan algebras. Mod. Phys. Lett., A8:1407-1416, 1993.

[HT95] C. M. Hull and P. K. Townsend. Unity of superstring dualities. Nucl. Phys., B438:109-137, 1995. [,236(1994)].

[JvNW34] Pascual Jordan, J. von Neumann, and Eugene P. Wigner. On an Algebraic generalization of the quantum mechanical formalism. Annals Math., 35:29-64, 1934.

[Kac80] V. G. Kac. Some remarks on nilpotent orbits. J. of Algebra, 64:190-213, 1980.

[Kna02] A. W. Knapp. Lie Groups Beyond an Introduction, volume 140 of Progress in Mathematics. Birkhäuser Boston Inc., Boston, MA, second edition, 2002.

[Kom90] B. P. Komrakov. Maximal subalgebras of real Lie algebras and a problem of sophus Lie. Soviet Math. Dokl., 41:269-273, 1990.

[KT83] Taichiro Kugo and Paul K. Townsend. Supersymmetry and the Division Algebras. Nucl. Phys., B221:357-380, 1983.

[LG72] M. Lorente and B. Gruber. Classification of semisimple subalgebras of simple Lie algebras. J. Math. Phys., 13:1639, 1972.

[Min06] A. N. Minchenko. The semisimple subalgebras of exceptional Lie algebras. Trans. Moskow Math. Soc., 236:225-269, 2006.

[MNY11] Ruben Mkrtchyan, Armen Nersessian, and Vahagn Yeghikyan. Hopf maps and Wigner's little groups. Mod. Phys. Lett., A26:1393-1405, 2011.

[MP81] W. G. McKay and J. Patera. Tables of dimensions, indices, and branching rules for representations of simple Lie algebras, volume 69 of Lecture Notes in Pure and Applied Mathematics. Dekker, 1981.

[MPRR17] Alessio Marrani, Gianfranco Pradisi, Fabio Riccioni, and Luca Romano. Nonsupersymmetric magic theories and Ehlers truncations. Int. J. Mod. Phys., A32(19n20):1750120, 2017.

[MR19] Alessio Marrani and Luca Romano. Orbits in Non-Supersymmetric Magic Theories. 2019. arXiv:1906.05830 [hep-th].

[Oni04] Arkady L. Onishchik. Lectures on Real Semisimple Lie Algebras and Their Representations. European Mathematical Society, Zürich, 2004.

[OV94] A. L. Onishchik and E. B. Vonberg. Lie Groups and Lie Algebras III. Springer Verlag, Berlin, 1994.

[Roz56] B. A. Rozenfeld. Geometrical interpretation of the compact simple Lie groups of the class e. Dokl. Akad. Nauk. SSSR, 106:600, 1956. (in Russian).

[Sla81] R. Slansky. Group Theory for Unified Model Building. Phys. Rept., 79:1-128, 1981.

[Sug59] Mitsuo Sugiura. Conjugate classes of Cartan subalgebras in real semi-simple Lie algebras. J. Math. Soc. Japan, 11:374-434, 1959.

[Tit66] J. Tits. Algèbres alternatives, algèbres de Jordan et algèbres de Lie exceptionnelles. I. construction. Nederl. Akad. Wetensch. Proc. Ser., A69:223, 1966. (in French).

[Yam15] Naoki Yamatsu. Finite-Dimensional Lie Algebras and Their Representations for Unified Model Building. 2015. arXiv:1511.08771 [hep-ph].

Dipartimento di Matematica, Università di Trento, Italy

E-mail address: degraaf@science.unitn.it

Museo Storico della Fisica e Centro Studi e Ricerche Enrico Fermi, Roma, Italy, and Dipartimento di Fisica e Astronomia Galileo Galilei, Università di Padova, and INFN, SEZIONE DI PADOVA, ITALY

E-mail address: alessio.marrani@pd.infn.it 